Florida International University FIU Digital Commons

$11-22-2004$

\title{
Design and development of a one-degree-of- freedom force-reflecting manual controller prototype for teleoperation
}

Chandrasekar Reddy Puligari

Florida International University, chandu_puligari@yahoo.com

DOI: $10.25148 /$ etd.FI08081538

Follow this and additional works at: https://digitalcommons.fiu.edu/etd

\section{Recommended Citation}

Puligari, Chandrasekar Reddy, "Design and development of a one-degree-of-freedom force-reflecting manual controller prototype for teleoperation" (2004). FIU Electronic Theses and Dissertations. 39.

https://digitalcommons.fiu.edu/etd/39 


\title{
FLORIDA INTERNATIONAL UNIVERSITY
}

Miami, Florida

\section{DESIGN AND DEVELOPMENT OF A ONE-DEGREE-OF-FREEDOM FORCE- REFLECTING MANUAL CONTROLLER PROTOTYPE FOR TELEOPERATION}

\author{
A thesis submitted in partial fulfillment of the \\ requirements for the degree of \\ MASTER OF SCIENCE \\ in \\ MECHANICAL ENGINEERING \\ by \\ Chandrasekar Reddy Puligari
}




\section{To: $\quad$ Dean Vish Prasad}

College of Engineering

This thesis, written by Chandrasekar Reddy Puligari, and entitled Design and Development of a One-Degree-of-Freedom Force-Reflecting Manual Controller Prototype for Teleoperation, having been approved in respect to style and intellectual content, is referred to you for judgment.

We have read this thesis and recommend that it be approved.

Ibrahim N. Tansel

Diana M. Rincón

Sabri Tosunoglu, Major Professor

Date of Defense: November 22, 2004

The thesis of Chandrasekar Reddy Puligari is approved.

\begin{tabular}{r}
$\begin{array}{r}\text { Dean Vish Prasad } \\
\text { College of Engineering }\end{array}$ \\
\hline Dean Douglas Wartzok \\
University Graduate School
\end{tabular}

Florida International University, 2004 


\section{DEDICATION}

I dedicate this thesis to my parents Mohan Reddy and Sukeshini without whom this work would not have been completed and to my brother Rajashekar Reddy who has been encouraging and supporting me. Without their patience, support, and most of all love, the completion of this work would not have been possible. I love you all. 


\section{ACKNOWLEDGMENTS}

I wish to thank my committee members Drs. Sabri Tosunoglu, Ibrahim Tansel and Diana Rincon for their support and patience. Especially, I would like to thank Professor Sabri Tosunoglu for advising and supporting me throughout the course of my research. Their guidance has been most helpful and appreciated.

My thanks are also extended to Dr. Daniel W. Repperger, Program Manager and Technical Contact for the U.S. Air Force grant (Grant No: 571859500 and Panther Soft Grant No: 212600507) supporting me throughout my graduate studies.

Furthermore, I would also like to thank my friends and peers Jordi Blanch, Andre Senior, Can (John) Dede, Ricardo Garcia, Satya, and Ravi K. Dowluri for their support and help in developing the software and conducting experiments. 


\begin{abstract}
OF THE THESIS
DESIGN AND DEVELOPMENT OF A ONE-DEGREE-OF-FREEDOM FORCEREFLECTING MANUAL CONTROLLER PROTOTYPE FOR TELEOPERATION

by
\end{abstract}

Chandrasekar Reddy Puligari

Florida International University, 2004

Miami, Florida

Professor Sabri Tosunoglu, Major Professor

The present research is carried out from the viewpoint of primarily space applications where human lives may be in danger if they are to work under these conditions. This work proposes to develop a one-degree-of-freedom (1-DOF) force-reflecting manual controller (FRMC) prototype for teleoperation, and address the effects of time delays commonly found in space applications where the control is accomplished via the earthbased control stations.

To test the FRMC, a mobile robot (PPRK) and a slider-bar were developed and integrated to the 1-DOF FRMC. The software developed in Visual Basic is able to telecontrol any platform that uses an SV203 controller through the internet and it allows the remote system to send feedback information which may be in the form of visual or force signals. Time delay experiments were conducted on the platform and the effects of time delay on the FRMC system operation have been studied and delineated. 


\section{I: INTRODUCTION}

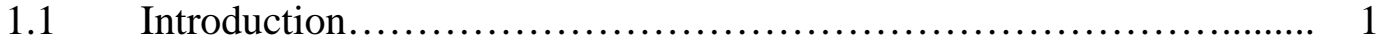

1.2 Teleoperation.................................................. 2

$1.3 \quad$ Telerobot................................................................ 3

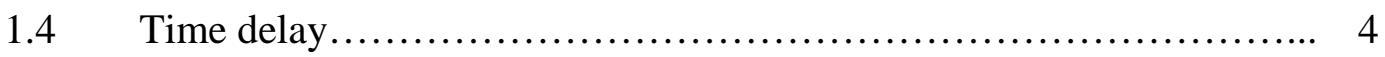

1.5 Thesis objective................................................ 5

\section{II: BACKGROUND AND DESIGN OF MANUAL CONTROLLERS}

$2.1 \quad$ History........................................................ 7

2.2 Force-reflecting manual controller (FRMC) system................. 10

2.3 Conceptual designs of manual controllers.......................... 15

2.3.1 FRMC with ball screw mechanism............................ 16

2.3.2 FRMC with gear system................................... 17

2.3.3 FRMC with belt system................................ 18

2.3.4 FRMC with direct drive mechanism........................ 19

2.3.5 FRMC with drive roller and belt system..................... 21

\section{III: ACTUATORS AND SENSORS}

$3.1 \quad$ Actuators ........................................................ 22

3.1.1 Hydraulic............................................. 22

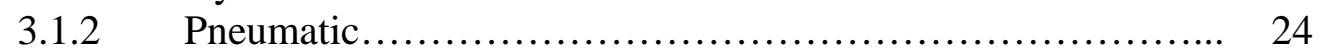

3.1.3 Electric................................................... 25

3.1.4 Selection................................................. 27

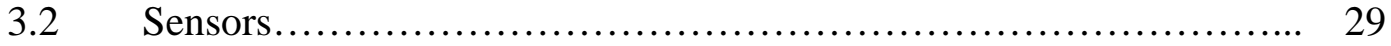

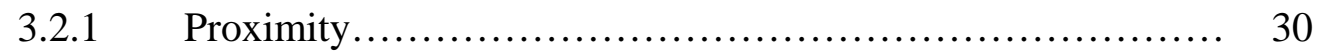

3.2.2 Range .......................................................... 30

3.2.3 Touch................................................. 31

3.2.4 Force/torque.......................................... 31

3.2.5 Potentiometer............................................... 32

3.2.6 Vision..................................................... 32

3.2.7 Tachometer............................................. 33

3.2.8 Selection............................................. 33

IV: MICROCONTROLLERS

4.1 Mini board................................................. 37

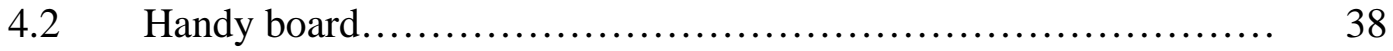

$4.3 \quad$ Bot board....................................................... 39 


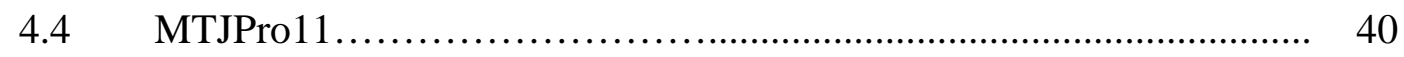

$4.5 \quad$ Basic stamp................................................... 41

4.6 Other microcontrollers............................................ 42

$4.7 \quad$ Servo controllers............................................... 43

4.7.1 Brainstem servo controller....................................... 43

4.7.2 Pololu servo controller..................................... 44

4.7.3 USB servo controller..................................... 45

4.7.4 Selection: Pontech SV203 servo controller..................... 46

\section{V: FRMC AND PLATFORM DESIGN}

$5.1 \quad$ Force-reflecting manual controller system design.................... 48

$5.2 \quad$ Power supply and pin out..................................... 51

$5.3 \quad$ Housing ........................................................ 52

$5.4 \quad$ Slider bar platform design....................................... 53

$5.5 \quad$ Palm pilot robot kit platform design............................. 54

5.5.1 Platform design modification............................. 55

\section{VI: ROBOT CONTROL SOFTWARE DEVELOPMENT AND SYSTEM} INTEGRATION

6.1 Robot control software....................................... 57

6.2 1-DOF FRMC Prototype simulation with mouse...................... 59

6.3 1-DOF FRMC Prototype simulation with SV203 controller........... 60

6.4 Time delay in space applications................................. 62

6.5 Time delay experiments conducted with 1-DOF FRMC.................. 64

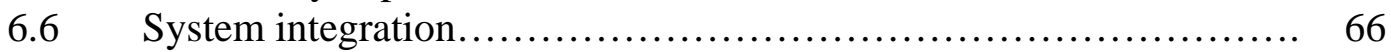

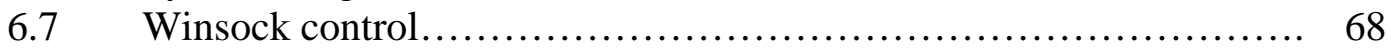

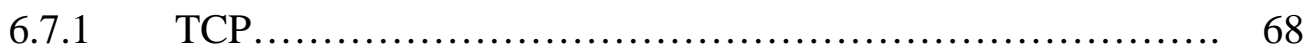

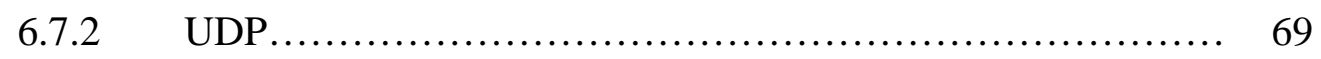

6.7.3 Winsock properties....................................... 69

6.7.4 Winsock methods....................................... 70

$6.8 \quad$ Operating the system software ....................................... 71

6.8.1 Video streaming........................................ 73

VII: RESULTS AND DISCUSSIONS

$7.1 \quad$ Conclusions.................................................. 76

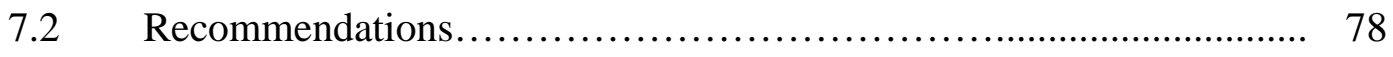

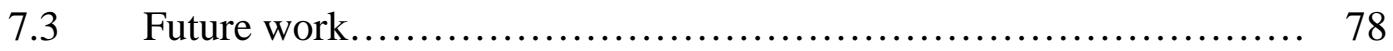

REFERENCES............................................................. 80

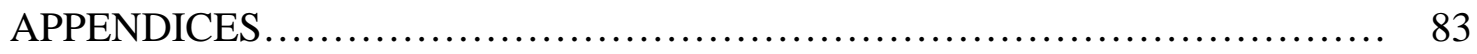




\section{LIST OF FIGURES}

FIGURE

PAGE

Figure 1.1 Telerobotic system block diagram.............................. 3

Figure 1.2 Telerobot working on mars.................................... 4

Figure 2.1 FRMC concept utilizing ball screw mechanism..................... 16

Figure 2.2 FRMC concepts with gear reduction............................ 18

Figure 2.3 FRMC concepts with belt system............................ 19

Figure 2.4 FRMC concepts utilizing direct drive system....................... 20

Figure 2.5 Roller drive and belt joystick............................... 21

Figure 3.1 Hydraulic actuator.......................................... 23

Figure 3.2 View of pneumatic actuator................................. 24

Figure 3.3 Servo timing diagram..................................... 28

Figure 3.4 Futaba servo motor....................................... 28

Figure 3.5 Non-linear graph.......................................... 34

Figure 3.6 GP2D12 sensor......................................... 34

Figure 3.7 Distance through triangulation................................ 35

Figure 3.8 Internal block diagram...................................... 35

Figure 4.1 PIC microcontroller......................................... 36

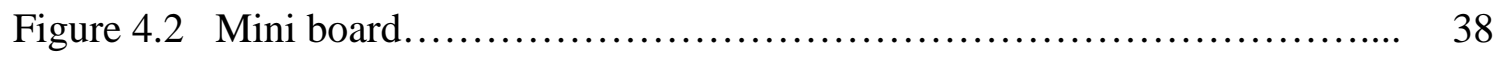

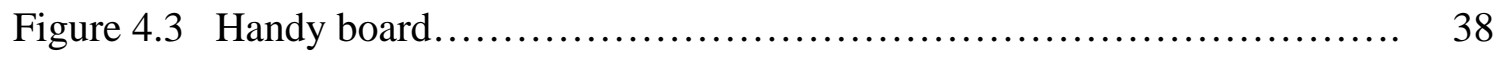

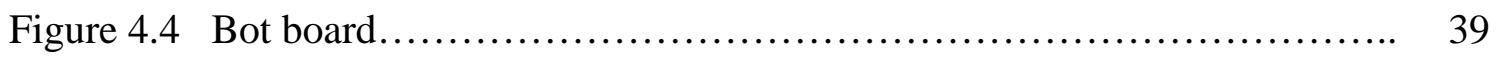

Figure 4.5 MTJPro11 microcontroller................................... 40

Figure 4.6 Basic stamp.............................................. 41 
Figure 4.7 AT89C2051 microcontroller................................. 42

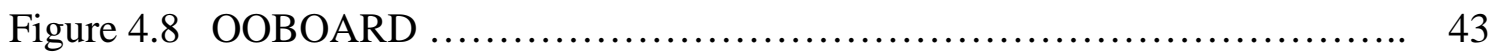

Figure 4.9 Brainstem controller........................................ 44

Figure 4.10 Pololu servo controller..................................... 45

Figure 4.11 USB servo controller........................................ 45

Figure 4.12 SV203 controller.......................................... 46

Figure 5.1 Direct drive mechanism................................... 49

Figure 5.2 Connection of servo with SV203............................. 50

Figure 5.3 Pin out diagram........................................... 51

Figure 5.4 Connection of potentiometer with SV203....................... 52

Figure 5.5 1-DOF FRMC developed.................................... 52

Figure 5.6 Slider bar platform........................................ 53

Figure 5.7 PPRK designed at CMU.................................... 54

Figure 5.8 PPRK modified mobile platform .............................. 56

Figure 6.1 Form view when robot on the left is away from the obstacle represented by the plane icon............................................ 59

Figure 6.2 Form view when robot is close to the obstacle..................... 60

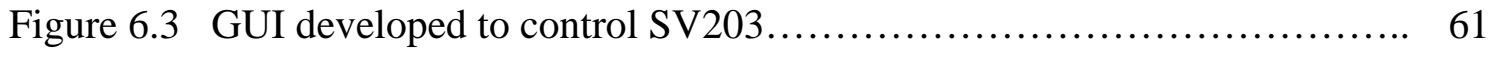

Figure 6.4 GUI of visual basic program................................. 62

Figure 6.5 Round-trip communication time delays between the earth and planets... 63

Figure 6.6 Time delay trend for different users identified as series 1 through $5 \ldots . .65$

Figure 6.7 Process of the system developed.............................. 72

Figure 6.8 Camera attached to the PPRK................................ 74 
Figure 6.9 Webcam at client......................................... 75

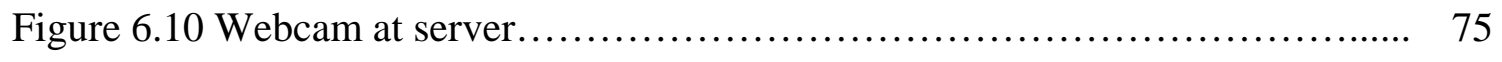




\section{CHAPTER 1}

\subsection{INTRODUCTION}

\subsection{Introduction}

The term robot is defined as a mechanical device that sometimes resembles a human being and is capable of performing a variety of often complex human tasks on command or by being programmed in advance. The term robota or rather robot means tedious labour in czech and was first used in Czechoslovakia by Karel Capek in a play called Rossum's Universal Robots (R.U.R) around 1920 [1] . Americans were the first to develop a programmable robot which was used to spray paint. This programmable robot was developed by Willard Pollard and Harold Roselund for a company named Devilbiss in 1938. In 1942, Isaac Asimov published three laws of robotics in a short story called "Runaround" [2]. The three laws of robotics are stated as follows:

1. A robot may not injure a human being or, through inaction, allow a human being to come to harm.

2. A robot must obey orders given it by human beings except where such orders would conflict with the First Law.

3. A robot must protect its own existence as long as such protection does not conflict with the First or Second Law.

The first teleoperated articulated arm was developed by Raymond Goertz in France around 1951 for the Atomic Energy Commission. The design is based entirely on mechanical coupling between the master and slave arms (using steel cables and pulleys). Derivatives of this design are still seen in places where handling of small nuclear samples 
is required. This is generally regarded as the major milestone in force feedback technology. The pioneering work by George Devol with the help of Joseph Engelberger led to the development of a programmable robot for industrial applications through the company named Unimation in 1954. This robot model was developed only for the basic use like transfer objects from one point to another, less than a dozen feet apart. The first industrial robot from Unimation was installed in the production line of General motors company around 1962. Day by day the use of robots became more popular but they also have a disadvantage in 60 's and 70's that they are a bit expensive, as the time passed by, they gradually became inexpensive due to their use in daily life. Car manufacturing companies have used these robots more than anyone else in those days for improving their production. In the present world the applications of the robots have been extended to laboratories, research and space exploration sites, energy plants, hospitals, toy industries and even to outer space.

\subsection{Teleoperation}

Teleoperation plays a vital role in the field of robotics. Teleoperation system usually consists of two robot manipulators that are connected in such a way as to allow the human operator controls one of the master arm ( one of the manipulator ) to generate commands which sends commands to the salve arm ( remote manipulator) which are kept at some distance apart. The commands from the master robot are sent to the slave robot by many different ways. Teleoperation has very wide range of application in the field of medicine, space exploration, military, undersea and hazardous applications etc. Perhaps the most common application of this technique is in the field of space 
exploration. In a typical teleoperation system, the operator (master robot) receives feedback information from the slave robot in any of the forms like audio, visual, and force [3]. Fig. 1.1 shows the basic principle behind a telerobotic system. This teleoperation is very useful in dangerous applications like nuclear waste cleanup sites where a human cannot fulfill the desired task.

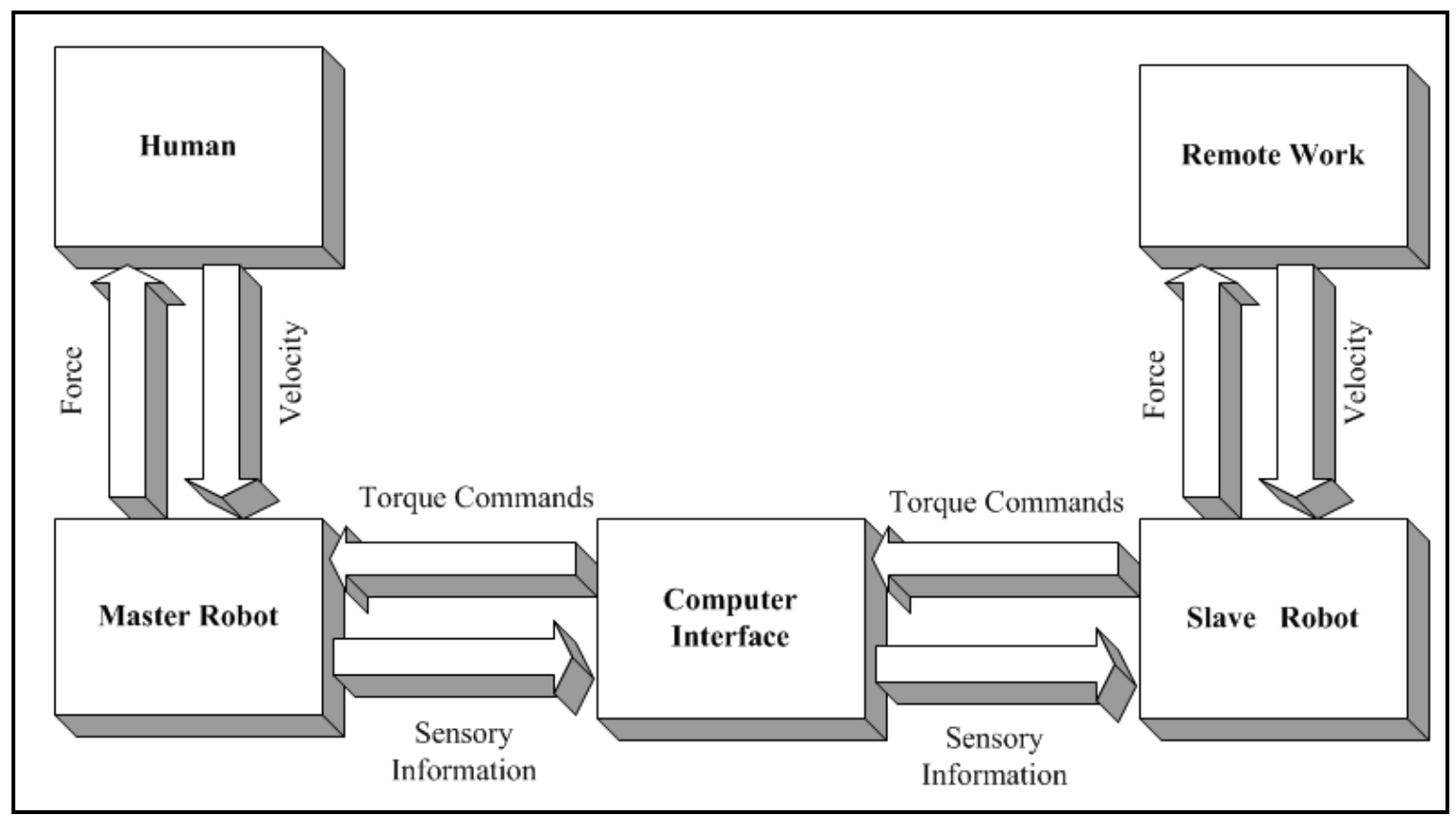

Figure 1.1 Telerobotic system block diagram

\subsection{Telerobot}

Latest technology in the field of robotics and automation has replaced many jobs that are repetitive in nature. However, there are many tasks that are non-repetitive, unpredictable and hazardous to human health. These tasks have to be performed by a remote manipulator, otherwise known as a telerobot. Telerobotics refers to the use of 
robots "at a distance". Latest development in the field of telerobot is the exploration of mars by National Aeronautics and Space Administration (NASA) is shown in fig 1.2. The applications of telerobots are wide spread in today's world such as remote control for televisions, music systems and also remote controls for garage doors etc.

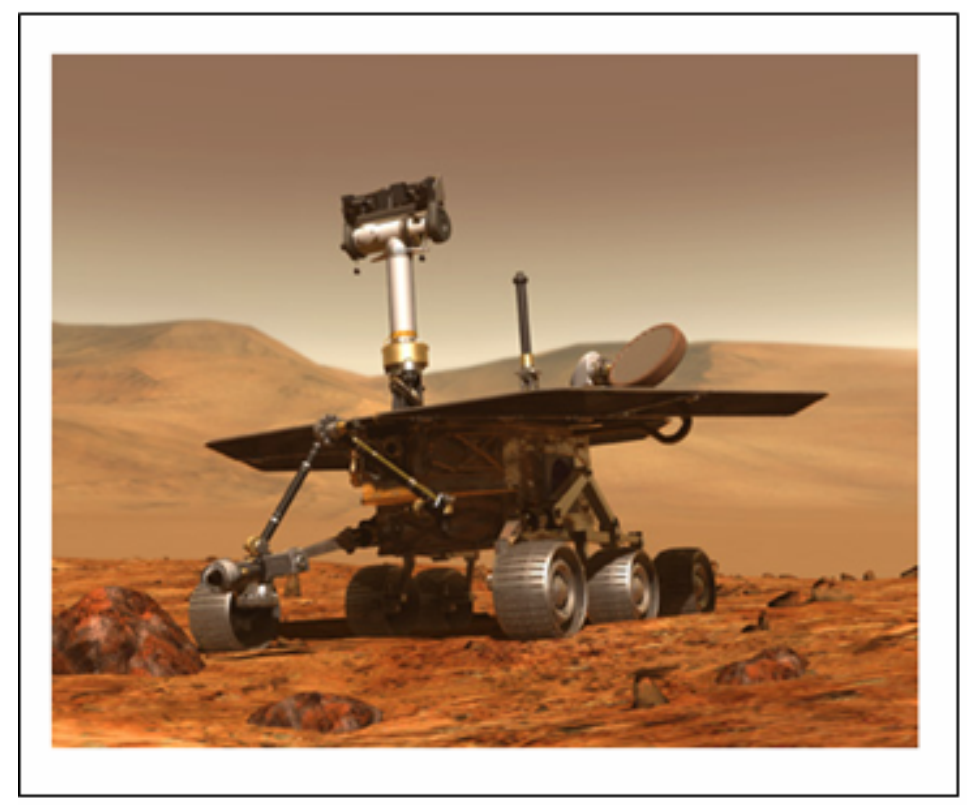

Figure 1.2 Telerobot working on mars

\section{$1.4 \quad$ Time delay}

In a teleoperation system there are two robots. A human operator moves and controls the local robot, also known as the input or master joystick. Motion commands are measured on this device and transmitted to remote location. The second robot, called slave, executes these commands and tries to track the input device. In many of the cases the time taken to execute the commands of the master robot by the slave robot are delayed due to some intermediate delays, these are called constant time delays. When Internet is used as the communication medium connecting the master and slave manipulators, where 
transmission delays are variable. For teleoperation over the Internet the delay varies with such factors as congestion, bandwidth, or distance, and these varying delays may severely degrade performance or even result in an unstable system. There has to date been relatively little research on this problem [18].

\subsection{Objectives of the current research}

One of the desired objectives in the development of the teleoperators systems is to design and develop a system that provides the operator with the sensation feedback. A force-reflecting manual controller is one of the components in the system that provides the operator with force feedback. Unfortunately, most of the manual controllers are large, bulky, complicated and expensive. For the system to be used in practical situation, the system must e portable, compact, lightweight, easy to use and easy to manufacture. Other requirements include large workplace and sufficient force reflection.

In this work, the design and development of a portable force reflecting manual controller is addressed as a design problem. This includes conceptual design of the system, construction of the testbed, software development, testing, and laboratory demonstration.

In order to satisfy above requirements, the previous works have been investigated for mechanical and control design. In addition, a survey of mechanical components such as actuator systems and sensors has been conducted to identify compact size, light weight, and high performance components. The proposed conceptual designs for a one degree of freedom (DOF) force reflecting manual controller (FRMC) have been shown and the developed prototype has been demonstrated on a 1-DOF testbed. 
The contents of each chapter are summarized as follows:

Chapter 1: History of robots is briefly described and the concept of teleoperators and the telesensation systems is introduced. Then, the objectives and the scope of the research work are outlined.

Chapter 2: Background on force-reflecting teleoperation systems is presented by first listing the history of the previous work. Conceptual designs of 1-DOF FRMC developed at FIU are discussed.

Chapter 3: The components of FRMC like actuators, sensors and potentiometers are discussed and the selection of these components are tabulated.

Chapter 4: The important component of a FRMC teleoperator system SV203 microcontroller and the other microcontrollers considered for this project are briefly described.

Chapter 5: The design and development of 1-DOF FRMC for teleoperation prototype and a platform (Palm Pilot Robot Kit) was developed to test the 1-DOF FRMC is discussed.

Chapter 6: The software developed to control this 1-DOF FRMC prototype is discussed. The graphical user interface and the simulation results are shown. The software developed is updated to control the joystick over the internet to test the teleoperation. The system networking is presented in this chapter.

Chapter 7: The conclusions and recommendations derived from this work, and future work to be conducted are presented. 


\section{CHAPTER 2}

\subsection{BACKGROUND AND DESIGN OF MANUAL CONTROLLERS}

Telemanipulator is a device which allows an operator to perform a task at a distance. Usually, a teleoperator system consists of one or more telemanipulators, task and environmental sensing systems, and a human machine interface. The applications of these telemanipulators include hazardous radioactive environment, underwater, space, military applications where human access is impossible.

In this chapter, previous work done by the researchers in the field of telemanipulator design will be described, and some of the previous teleoperation system designs are reviewed.

\section{$2.1 \quad$ History}

- In 1945, Ray Goertz developed the first manual controller at Argonne National Laboratory (ANL). The first developed manual controller does not include force reflecting feature in it but later they have implemented it.

- In 1949, the first Master-Slave Manipulator (MSM) was developed by Ray Goertz and his team at ANL of the U.S. Atomic Energy Commission. It was given the name Model 1; this bilateral manual controller uses electrical and mechanical systems to penetrating through the ceiling of a shielded cell. Maximum handling capacity was only $5 \mathrm{kgs}$.

- In 1953, there was a need to design a manipulator with high handling capacities, which inturn resulted in developing Model 8 manipulator with a handling capacity of 
9 kgs. This was developed by Central Research Laboratory (CRL) under the supervision of ANL. CRL has manufactured $60-70 \%$ of the existing Model 8 manipulators.

- In 1954, Goertz and Thompson have replaced the direct mechanical linkage and cable linkages with electrical servomechanisms and developed a closed circuit television, which resembles a teleoperation where the operator could be an arbitrary distance away.

- In 1960, CRL has developed Model D manipulator. It is the first manipulator used for some industrial application with higher load capacity after all the models developed for only laboratory application. The improved versions of these Model D manipulators are Model E and Model F.

- In 1960, R. Mosher developed a Handy man at General Electric. This is a force reflecting manual controller which has two electro hydraulic arms with ten degrees of freedom (each finger has two degrees of freedom).

- In 1961, Aaron Kobrinskii in Moscow was the first to develop the new servomechanism to human limb prostheses. He developed a lower-arm prosthesis driven by minute electric signals picked up from muscles in the stump or upper arm.

- In mid 1960's, Researches in United States and Europe have made similar developments as Aaron Kobrinskii. They developed teleoperators attached to the wheelchairs of quadriplegics which can be commanded by tongue.

- In 1966, Strickler was the first to develop teleoperator with teletouch.

- In 1966, telemanipulators and video cameras were attached to submarines by US navy. 
- In 1967, Ferrell and Sheridan developed the first teleoperator with time delay.

- In 1969, NASA developed a space teleoperator for space shuttle, remote manipulator system (RMS) which is $20 \mathrm{mts}$ long.

- In late 1960's, Telediagnosis was developed between a general hospital in Boston and Logan Airport by Murphy, R.L. Jr. and Bird K.T.

- In 1970's, Remotely Operated Vehicles have been developed for undersea applications by Vadus (1976), Yastrebov and Stepanov (1978), and Busby (1979).

- In 1981, M-2 Maintenance system and Advanced Servo manipulator (ASM) masterslave force reflecting manual controller for a nuclear plant was developed by Oak Ridge National Laboratory.

- In 1980's, Whitney at Draper Laboratory at Massachusetts Institute of Technology has developed handy controller.

- In 1985, Tesar and Tosunoglu at University of Texas, Austin have developed both 6DOF force reflecting manual controller and a 3-DOF spherical force reflecting shoulder controller.

- In 1993, Massachusetts Institute of Technology Artificial Intelligence Laboratory has developed a virtual force feedback master controller PHANToM which was made commercially available.

- In 1993, EXOS Inc. developed commercially available force reflecting manual controller named SAFiRE (Sensing and Force Reflecting Exoskeleton).

- In 1996, Virtual Technologies Inc. developed a force feedback glove, where force reflection was experienced by each finger. 
- In 1998, Batsomboon and Tosunoglu have developed a portable force-reflecting manual controller for teleoperation at Florida International University.

- In 1999, a pen based force feedback device PenCAT was developed by Haptic Technologies Inc.

- In 2000, Michael Goldfarb at Vanderbilt University has developed 3-DOF haptic interface in Centre for Intelligent Mechatronics.

- In 2002, Howe and Brockett at Harvard Robotics Lab have developed a HRL manipulator which has 2 two link fingers, each with 3-DOF.

- In 2004, Kamerkar and Kesavdas at University of Buffalo developed a touch based interactive NURBS modeler using a force/position input glove.

- In 2004, Niemeyer, and Kuchenbecker at Stanford University developed a THUMP force reflecting device.

\section{$2.2 \quad$ Force-reflecting manual controller system}

Force Reflecting Manual controllers are used in telerobotic applications to control remote robots by local human operators. The applications include space operations, undersea activities, nuclear site cleanup, and microsurgery. Usually the force-reflecting manual controller system consists of two robots, slave and a master. The operator uses the manual controller (master) to control the other robot (slave). The slave robot receives the signal from the master through various communication channels. When the master and slave robots are operated in two different locations visual information from a video image and/or graphical information from the computer screen or the sensory feedback are used to improve the efficiency of the manual controller. When the input 
commands are fed to the master, remote system moves accordingly and the force experienced by the system are reflected at the manual controller, which shows that the system is a force reflecting teleoperating system [15].

These force reflecting teleoperating systems are more helpful in a situation where human cannot complete the task due to some hazardous environments, and where the visual information of the remote site cannot be obtained. In such environments, this manual controller can be used effectively to complete the required task. The operator with force reflection tends to make fewer attempts to complete the given task than the operator without force reflection [4].

Some of the well known force reflecting manual controllers or Haptics developed as discussed below:

\section{- PHANToM}

PHANToM is the force reflecting desktop device developed by the MIT researcher Massie, a 3D input device which can be operated by the finger tip [5]. This is a 3-DOF force reflecting device that provides force reflection to the human operator when it's connected to the computer interface. This FRMC enables the user to feel the virtual reality environment. Different models of this PHANToM have designed like Desktop PHANToM device and PHANToM Omni device. These are considered to be high precision instruments and also some of these are 6-DOF controllers. This PHANToM offers high fidelity, stronger forces and low friction. This device produces a maximum force reflection of $7.9 \mathrm{~N}$ and a continuous force of $1.75 \mathrm{~N}[6]$. 


\section{- Rutgers master II ND}

This device is a force reflecting haptic interface which uses a glove to cover the fingers of a human instead of using fingers directly. This haptic interface has all the sensing devices on the glove avoiding routing wires to fingertips as in earlier devices. This uses the direct drive mechanism to feel the force reflection. This device uses the pneumatic actuator which is arranged in direct drive mechanism to the fingers. The glove which is covering the fingers will feel the force reflection after receiving the commands from the sensors attached to it, resulting in better force control [7].

\section{- GROPE-III}

The University of North Carolina (UNC) researches have modified the mechanical controller of a robotic arm previously used in radioactive material handling. Motors were added to the Argonne remote manipulator (ARM). The motors would be activated by the virtual environment to create forces on the controller. UNC used this in their GROPE-III system to help chemist "feel" the attractive and resistive forces of molecules reacting and bonding to each other. The chemist could use the forces and torques to learn how to make new chemical compounds.

\section{- Exoskeleton}

In 1988, University of Utah with collaboration with EXOS Company has developed a device for telerobotic application where [8], an operator can strap their arm into a large 50 pound exoskeleton developed to deliver force feedback. The systems are used as a kind of master-slave combination, and forces are applied by motors at the joints. The operator's arm and hand can move in 10 different ways at the same time. 
The computer constantly changes the force output of the motors and hydraulic actuators on the exoskeleton so that it can feel essentially weightless. However, when the operator touches something, the virtual forces become actual forces felt through the exoskeleton. They could feel the increasing resistance of compressing a virtual spring or their arm would stop hard when it reached a virtual wall. . Unfortunately, these devices are usually very heavy; therefore they can also be used in special applications. EXOS, Inc. has developed a "light" version for the NASA, but this system does not have any force feedback.

\section{- Force-reflecting hand controller}

A force reflective hand controller (FRHC) is being developed to control a robot arm. When the robot arm picks up an object or pushes something, those forces and torques are felt by the operator. The operator can now tell how much strain is placed on the robot's motors, gears, and structure. Also this controller gives the operator a partial sense of touch that the robot would have.

\section{- Touch based interactive NURBS modeler}

The creation of complex NURBS surfaces in design environments is a tedious process because very few tools exist, that allow a designer to design intuitively in real-time; to overcome this problem researchers at University at Buffalo (UB) have developed a device. Here in this design the human hand is perhaps the most useful and diverse tool used to interact with the environment and us. The CAD Modeling Glove (ModelGlove), originally developed by Mayrose (Mayrose et al. 2000) in the Virtual Reality Lab. At UB was adapted as an innovative new interface between the real world and the 3D NURBS model, by capturing the action of the user's hand including pressure and position of the 
fingers. This input device is equipped with force and position sensors for quantifying touch and intent of the designer. The goal behind the development of the ModelGlove is to provide designers with a tool, which will allow them to touch, push, and manipulate virtual objects, just as they would model real clay models or sculptures [9].

\section{- Pen based force display}

The pen based force display is a direct drive mechanism haptic device developed by University of Washington researchers. This device is a parallel, actuation redundant, two degree-of-freedom haptic device, designed to provide force feedback information generated by either a master-slave system or a virtual simulation. The operator interacts with it using either the fingertip or a freely held pen-like tool. This device could provide force feedback for applications such as micro-surgery and telemanipulation, or serve as an input device to characterize the human's finger impedance, or serve as a generic virtual reality mechanical interface [10].

\section{- Two-hand universal master project (THUMP)}

THUMP is a 3D manipulator used to operate the slave robot for precise robotic applications, developed by the Stanford researchers. This device consists of two hands operated structures resembling a human hand and fingers working in a 3D environment, which will be helpful to be considered as a robot working in virtual reality environment. Each arm of the robot manipulator has seven degrees of freedom to serve as a haptic interface. The two handed haptic capability will provide force and torque feedback identical to those experienced from the remote site. This device is will be much helpful in surgical applications [11]. 


\section{- Portable dextrous master}

The Portable Dextrous Master (PDM) is a force feedback system for delicate hand motions. It was designed and manufactured through a colaboration between Sarcos Inc., the Center of Engineering Design at Utah and the Artificial Intelligence Lab at MIT. It is designed to be used with the DataGlove. The DataGlove detects finger movement to the computer which then sends signals to the PDM which, in turn, supplies force feedback in the thumb, forefinger, and middle fingers [12].

\subsection{Conceptual designs of one-degree-of-freedom force-reflecting manual controller}

Many design constraints arise from consideration of the mechanical interaction of the human with the haptic interface. If the device is designed to be manipulated by the user's fingers, then the design parameters will be related to the capacity of a typical human finger. Interface size and appearance will also play vital roles in haptic interface design. In particular we want users to be instantly comfortable with our design. We believe that we can satisfy this goal with a 1 D.O.F device whose size and motion are similar to that of a PC mouse. The next section will consider how four interface proposals accomplish the design goals mentioned above. 


\section{Interface designs}

\subsubsection{FRMC Concept utilizing ball screw mechanism}

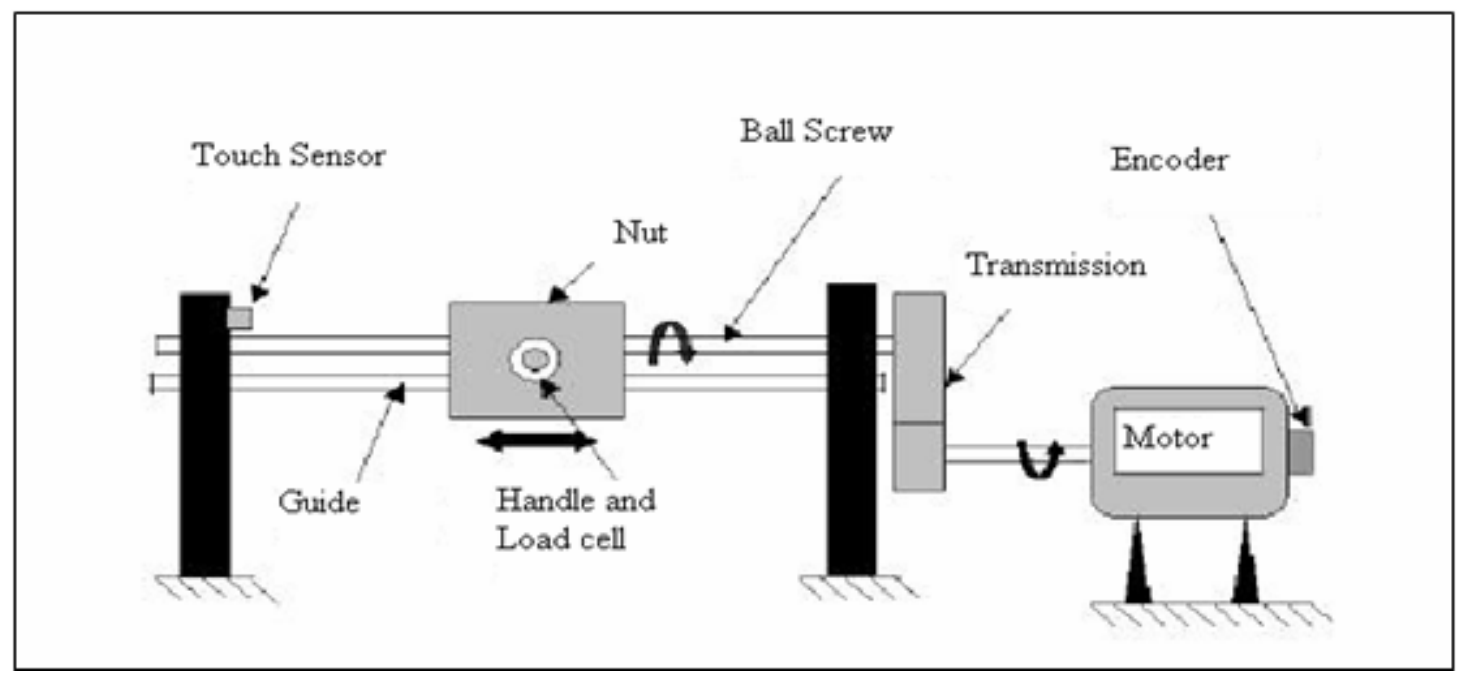

Figure 2.1 FRMC concept utilizing ball screw mechanism

A schematic of the ball screw joystick design is shown in Fig. 2.1. It consists of a ball screw $(9.5 \mathrm{~mm}$ in diameter, $12.7 \mathrm{~mm}$ lead), a fingertip handle attached to the nut, and a motor. A touch sensor is used to initialize the system and an encoder to measure the position of the handle. Ball Screws replace the sliding friction of the conventional power screw with the rolling friction of the bearing balls thereby reducing the friction between the screw threads and the mating nut. The low friction inherent in a ball screw makes them virtually non-self locking and back drivable.

With a desired linear speed of $0.3048 \mathrm{~m} / \mathrm{sec}$ of the nut, the rpm and the torque of the motor were calculated as $1440 \mathrm{rpm}$ and $0.068 \mathrm{Nm}$, respectively. Therefore Maxon EC $\phi 40 \mathrm{~mm}$, brushless 120 Watt motor with maximum continuous torque of $0.101 \mathrm{Nm}$ was 
considered. To determine the dynamic range of the actuator, the maximum and minimum forces are calculated as follows:

With the efficiency (E) of the lead screw of $78.91 \%$, lead (L) of the screw and maximum continuous torque $(\mathrm{T})$ of the motor, the maximum force $\left(\mathrm{F}_{\max }\right)$ of $39.2351 \mathrm{~N}$ was calculated using the relation $\mathrm{F}_{\max }=\left(2 * 3.1416^{*} \mathrm{E}\right) * \mathrm{~T} / \mathrm{L}$. Using the frictional torque of the motor of $0.0096 \mathrm{Nm}$ and breakaway torque of ball screw of $0.0212 \mathrm{Nm}$, the minimum force $\left(\mathrm{F}_{\min }\right)$ was $12.0158 \mathrm{~N}$.

The low dynamic range $\left(\mathrm{F}_{\max } / \mathrm{F}_{\min }\right)$ of 3.3 is a result of the large amount of friction inherent in the ball screw. For example, the ball screws reported breakaway torque is almost 2 times the friction torque of the motor. Since the low dynamic range would adversely affect the range of stimuli for the display, the ball screw joystick was taken out of consideration for our master controller.

\subsubsection{FRMC Concept with gear system}

The process of power transmission here is as follows: When the motor is switched on, the motor shaft rotates which is coupled to the pinion, which transmits power to the gear. The handle of the controller (joystick) is attached to the shaft of the pinion, which receives the transmitted power from the first gear. Fig 2.2 above describes the gear transmission system.

For this FRMC concept to be compact, we have to build the system by taking the gear ratio into account. For the FRMC to be successful, it should have an acceptable level of force reflection capability. Reducing the speed, which is accomplished by selecting a suitable gear ratio, will increase the torque output of the gear system. 
Our 1-DOF FRMC prototype will be capable of providing a maximum force reflection of about $5 \mathrm{lb}$. To be compact, the gears are selected with a gear ratio of $284: 1$, which reduces the output speed by 284 times which in turn magnifies the output torque of the gear system. Maximum speed of the motor shaft is $15052 \mathrm{rpm}$ and the speed at the output shaft will be reduced to a maximum speed of $53 \mathrm{rpm}$.

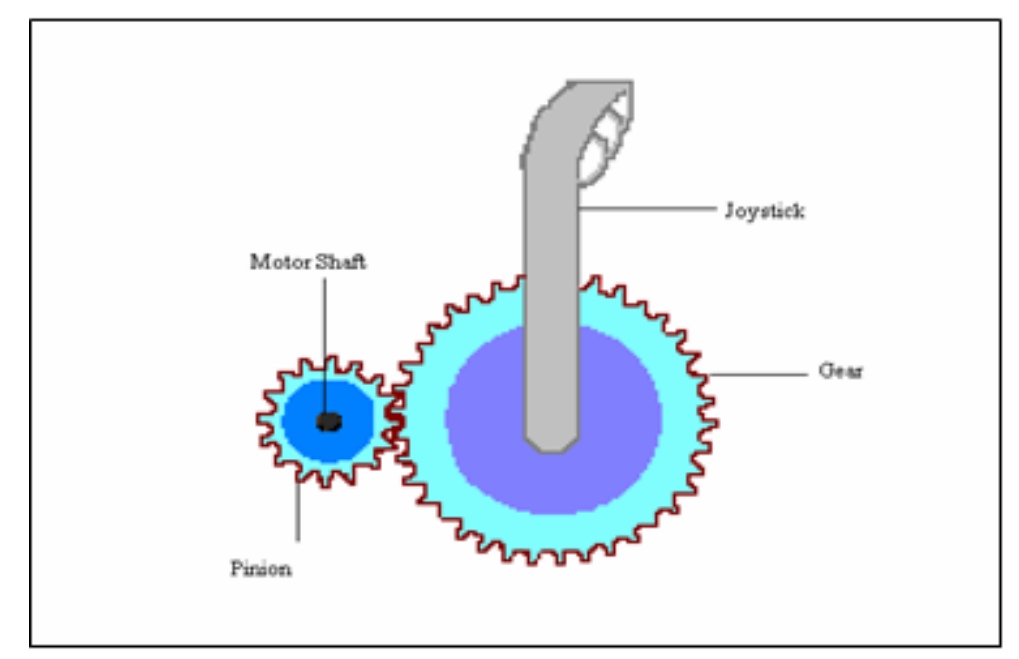

Figure 2.2 FRMC concepts with gear reduction

If we use spur gears, then the backlash will be more and the friction will be less relative to helical gears. If we use helical gears, we will have low backlash and the friction will be more when compared to the spur gears, there will not be any difficulty of back drivability in the case of spur gears.

\subsubsection{FRMC Concept with belt system}

The process of power transmission here is as follows: When the motor is switched on, the motor shaft rotates which is coupled to the pulley and it transmits power to the other pulley, which is connected to the joystick of the FRMC. The handle of the controller 
experiences the torque and the maximum force reflection that can be felt will be about 5 lb.

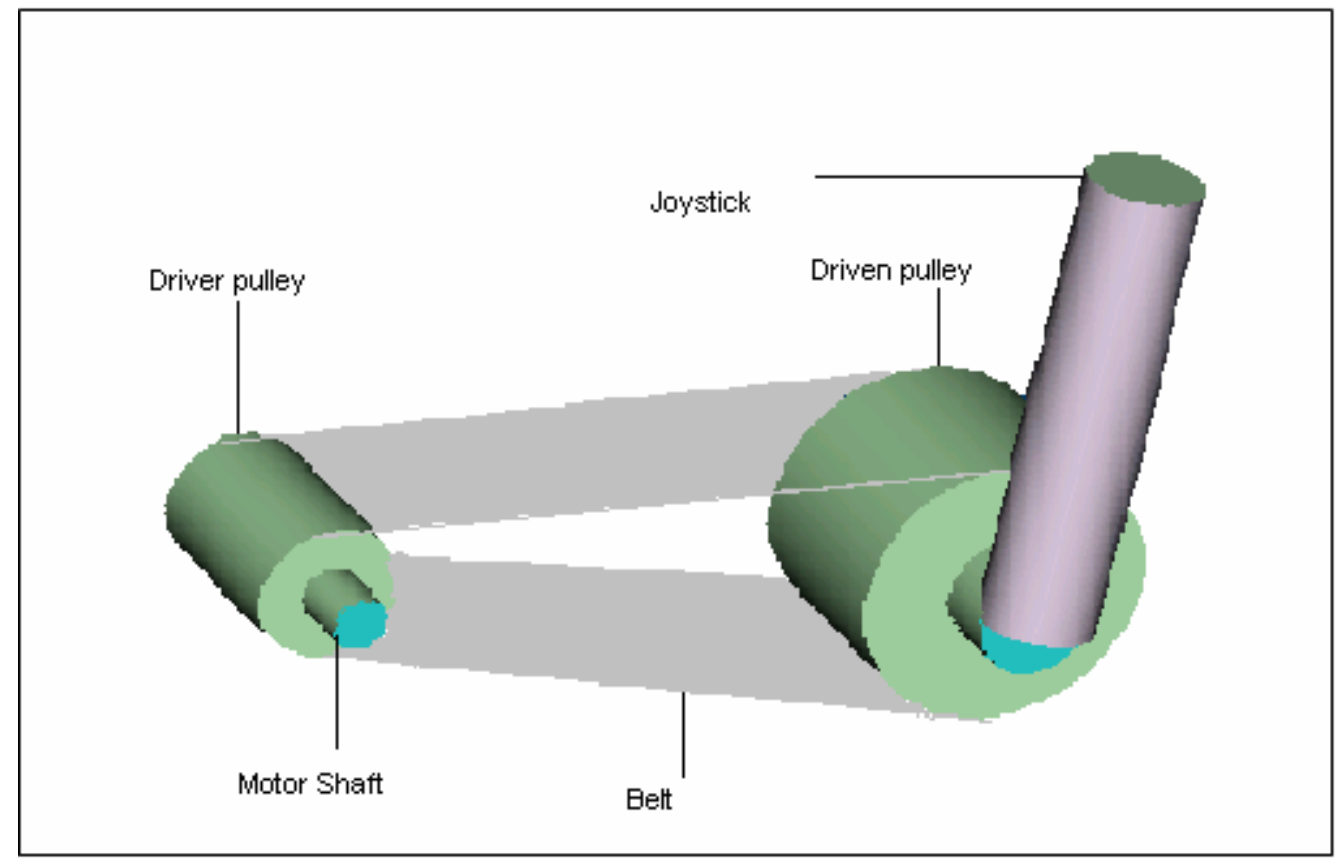

Figure 2.3 FRMC concepts with belt system

The main disadvantage with this concept of FRMC is that the slip factor plays a major role in reducing the torque and the force reflection will also be reduced do this slip factor. Distance between the pulley shafts is also a major limitation in this case. Belt drives are normally used to transmit power between relatively long distances. The Fig 2.3 shows the belt transmission system.

\subsubsection{FRMC Concept utilizing direct drive system}

In this concept, the joystick is directly attached to the actuator's shaft. By using this concept of direct drive method in FRMC makes the FRMC more compact and also more 
efficient as it directly couples to the joystick. The maintenance of this FRMC is inexpensive and the total cost for building this FRMC is also less compared to the other methods. By directly coupling the motor shaft to the joystick we can have acceptably high torque and also smooth operation. The only disadvantage here is that a more complex amplifier design may be required. A FRMC model with direct drive mechanism is shown in fig 2.4 .

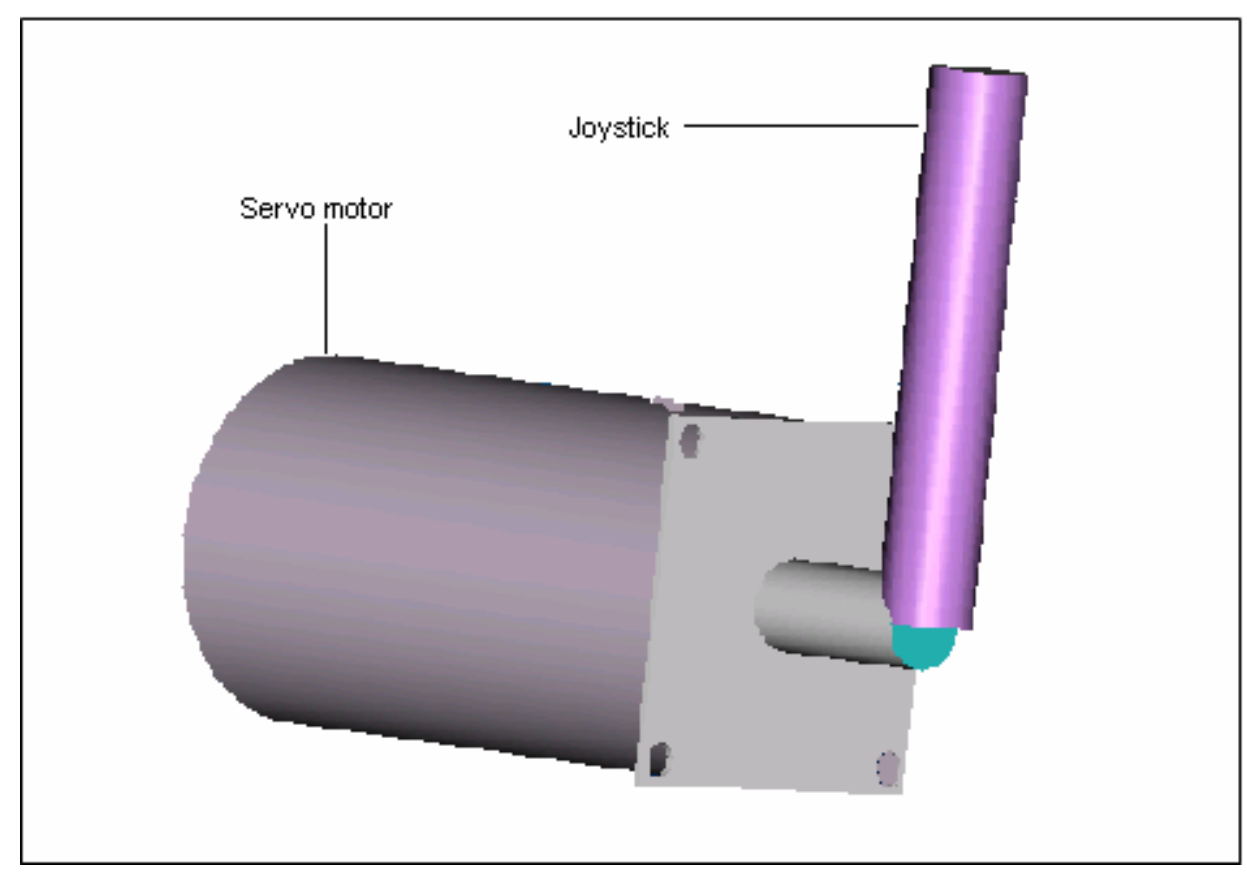

Figure 2.4 FRMC concepts utilizing direct drive system

Due to the direct linking of joystick to the motor shaft we don't have any intermediate losses. We also don't have any frictional losses or backlashes in this case. Unlike the gear transmission and belt transmission systems, direct drive system doesn't reduce speed and 
also there is no torque amplification. So we have to select such a motor that is suitable for this configuration; i.e., which has less speed and high torque.

\subsubsection{FRMC Concept with drive roller and belt system}

To combine direct drive with a more ergonomic design, a linear manipulator was also considered. As shown below in fig 2.5, the design consists of a handle, a linear bearing, motor, and two timing belt pulleys $(2.32 \mathrm{~cm}$ in diameter, 25 teeth, and $22.0 \mathrm{~cm}$ between pulleys). The motor chosen is the Maxon EC $\phi 45 \mathrm{~mm}$ brushless $250 \mathrm{~W}$ motor with a maximum continuous torque of $0.306 \mathrm{Nm}$

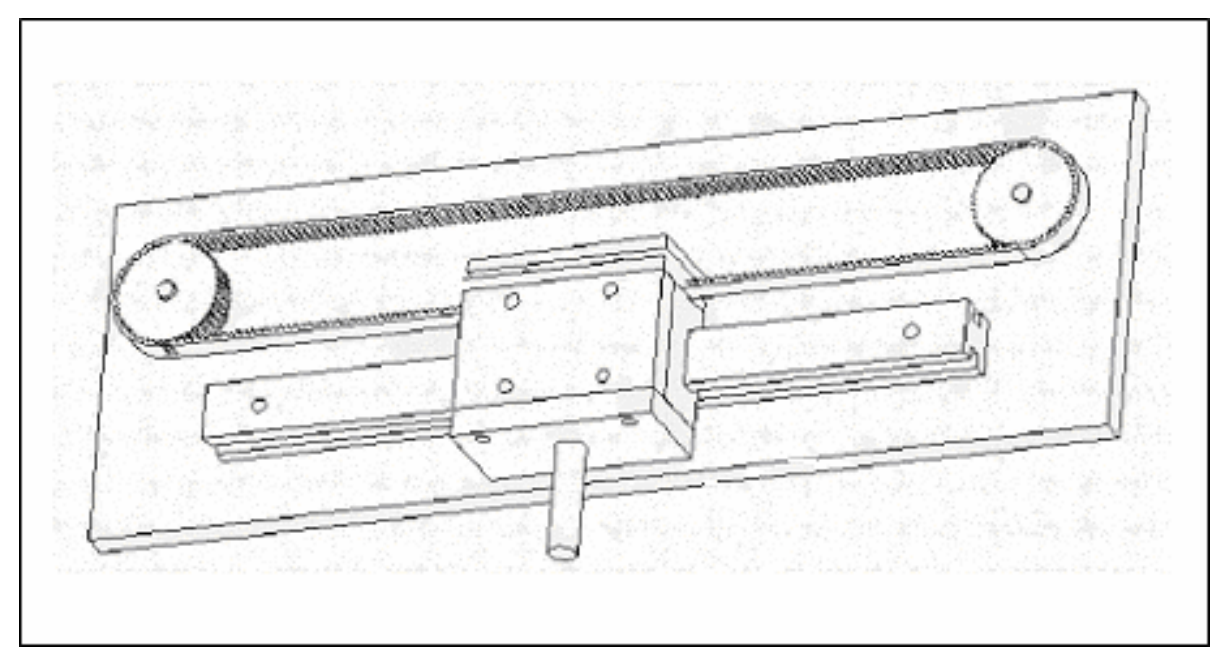

Figure 2.5 Roller drive and belt joystick

If a frictional (minimum) torque of $0.0206 \mathrm{Nm}$ and a maximum continuous torque of $0.306 \mathrm{Nm}$ of the motor are used, then a dynamic range of 14.8 is obtained. Since this design is suitable for ergonomic desktop manipulation and has a reasonable dynamic range, it may be a viable option for our haptic interface design. 


\section{CHAPTER 3}

\subsection{FORCE-REFLECTING TELEOPERATION SYSTEM COMPONENTS}

This chapter briefly describes the major components used in a force-reflecting teleoperation system. The components listed in this chapter are actuators, various sensors including potentiometers, sonars, IRs and tactile sensors. The following chapter is then dedicated to microprocessors used in robot design.

\subsection{Actuators}

Actuator is a device that produces a change in its shaft position (displacement) when a signal is applied to it. The input power for a force-reflecting teleoperation system is provided by the actuator. Usually there are two types of motion, linear and rotary depending on the actuator [14]. Different types of actuators are briefly described below.

\subsubsection{Hydraulic actuators}

Hydraulic actuators convert fluid power into motion for many robot applications and are normally used when a large amount of payload capacity is required. Although hydraulic actuators come in many designs, piston types are most common. A typical piston-type hydraulic actuator is shown in fig 3.1.

It consists of a cylinder, piston, spring, hydraulic supply and return line. The piston slides up and down inside the cylinder and separates the cylinder into two chambers. The upper chamber contains the spring and the lower chamber contains hydraulic oil. The hydraulic supply and return line is connected to the lower chamber and allows hydraulic fluid to flow to and from the lower chamber of the actuator. The stem 
transmits the motion of the piston to rest of the machinery. Initially, with no hydraulic fluid pressure, the spring force holds the valve in the closed position. As the fluid enters the lower chamber, pressure in it increases.

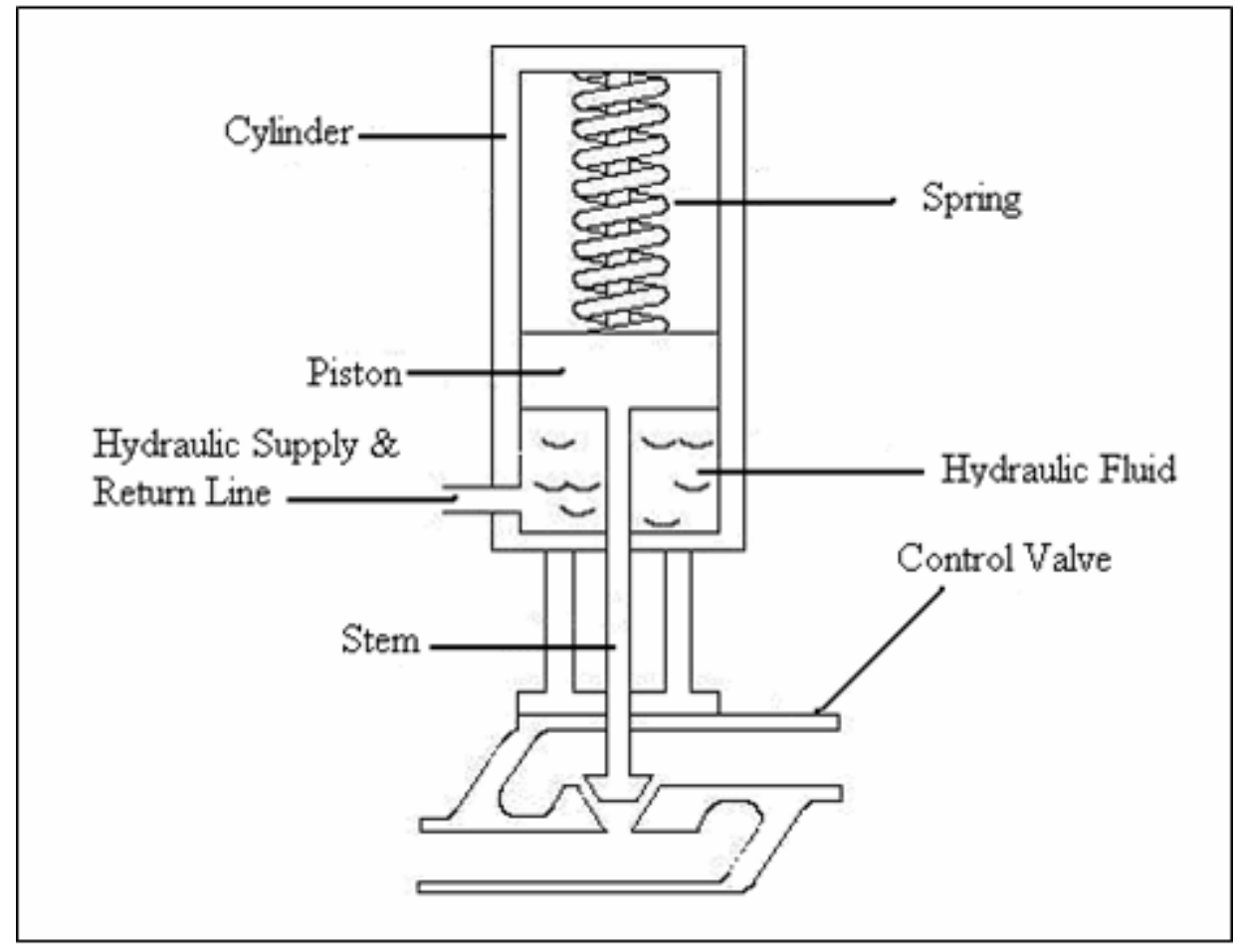

Figure 3.1 Hydraulic actuator

This pressure results in a force on the bottom of the piston opposite to the force caused by the spring. When the hydraulic force is greater than the spring force, the piston begins to move upward, the spring compresses. As the hydraulic pressure increases, the valve continues to open. Conversely, as hydraulic oil is drained from the cylinder, the hydraulic force becomes less than the spring force, the piston moves downward, and the valve closes. By regulating amount of oil supplied or drained from the actuator, the valve can be positioned between fully open and fully closed. The main advantages of these 
hydraulic actuators are self lubricating, self cooling, not flammable and smooth in operation while the main disadvantages are that they need maintenance, not good at high speeds, oil leakage problems, large space requirements and not back drivable [2].

\subsubsection{Pneumatic actuators}

Pneumatic drive systems are found in approximately 30 percent of today's robots. These systems use compressed air as the medium of energy transmission. With pneumatic actuators, the pressure within the chambers is lower than that of hydraulic systems resulting in lower force capabilities. In fig 3.2 there is a cut-away view of the basic pneumatic actuator. It is quite similar to the hydraulic counterpart; however, there are no return hydraulic lines for fluid. In a typical actuator of this type, the fluid, namely the air, is simply exhausted through the outlet valve in the actuator.

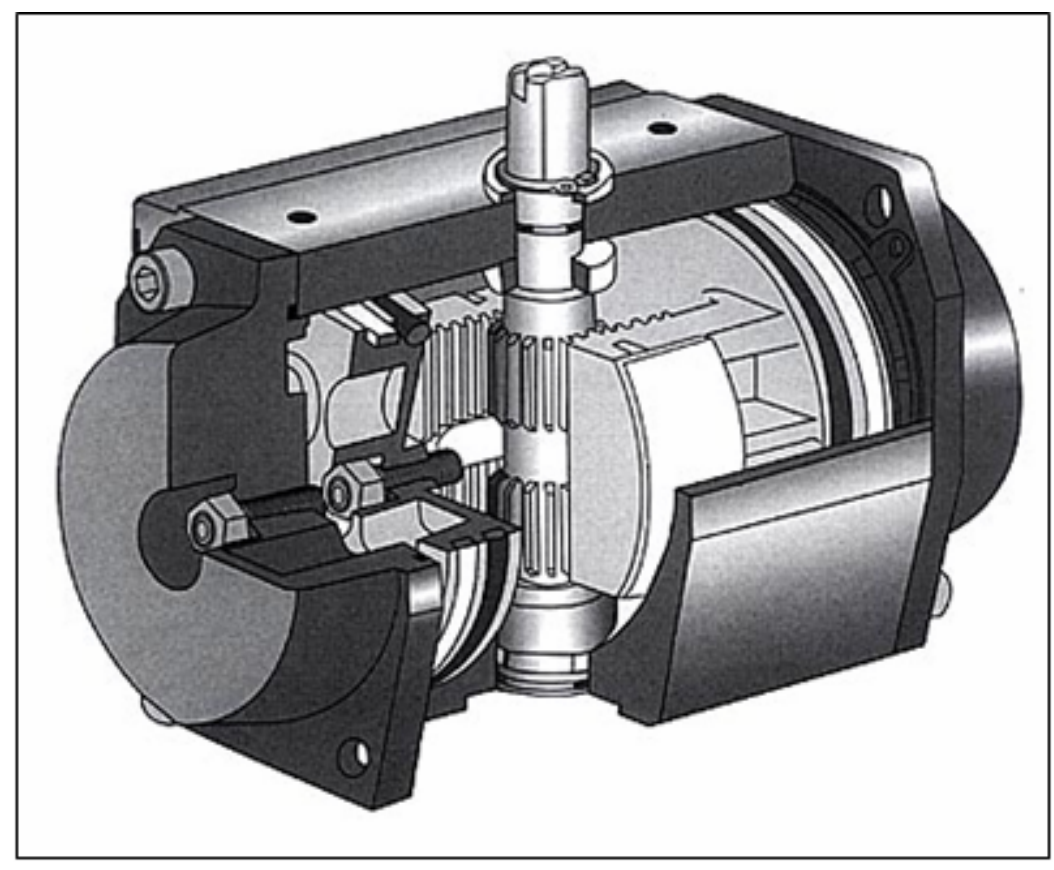

Figure 3.2 View of pneumatic actuator 
Pneumatic systems have been used in robotic systems when lightweight, small size systems are needed with relatively high payload-to-weight ratio. The main advantages of these hydraulic actuators are that they are non-flammable, suitable for clean environments, and easy to operate and maintain while the main disadvantages are that they do not have self lubricating properties and not suitable for harsh environments. Also, compressibility of air is seen as a disadvantage in view of the compressibility of air since it prevents easy control of speed and position of a robotic device, which are considered to be essential ingredients for any successful robot operation.

\subsubsection{Electrical actuators}

Electrical actuators are found in approximately 70 percent of the robotic applications. The principle used behind an electric motor is simple: A magnetic field is applied to a ferrous core, which induces motion. These electrical motors are used where the conversion of electrical energy into mechanical energy is essential. The main advantages of electrical actuators are their high reliability, low friction, fast, accurate, clean, and quiet operation, low maintenance, and low cost. They can also be easily controlled by a microprocessor or computer, which makes them a very attractive alternative.

While the main disadvantages are their high speeds with relatively low torques, which necessitates the use of gear trains or other power transmission systems. Another problem may arise if electric motors are over heated which may cause electrical arcing. Hence, they may not be suitable for environments where flaming is a concern. There are different types of electrical motors depending upon the way they are controlled [2]. These motor types are briefly reviewed below. 
- DC motors: when the voltage is applied to the terminals of the motor through armature then the motor begins to rotate. A DC motor is intended to work from a direct power supply. As the armature picks up speed, a voltage is induced in the winding that tries to oppose the current flowing in them. The speed at which balance is established (stabilization of motor speed) is a function of the applied voltage and the motor characteristics. The amount of turning force, torque that the motor can produce is a function of the current through the windings. DC motors respond quickly to changes in control signals due to the DC motor's high ratio of torque to inertia.

- $\quad$ Stepper motors: Stepper motor consists of two parts stator (fixed part) and rotor (rotating part). These motors are driven by a train of electrical pulses. The stator is wound as two separate coils, these coils are pulsed alternatively to produce a rotating magnetic filed. Each pulse turns the rotor through fixed angle; hence angular position change is proportional to the number of pulses. Stepper motors have a fixed number of magnetic poles that determine the number of steps per revolution. Some of the important features of the stepper motors are large torques, excellent rotational accuracy, compact size and works well for range of speeds.

- Servo motors: Servo motors have feedback mechanism which enables it to be closed loop operation. These actuators have an optical encoder which measures position and velocity of the motor shaft. These motors continuously monitors the position and velocity information and compares it with the desired values and makes necessary 
corrections to reach those desired values. These motors are bulkier and expensive. These motors have longer life and better heat dissipation properties.

- RC servos: These servos are very small in size and are inexpensive when compared to other motors. These Servos use potentiometer as feedback to determine their position, developed by hobby industry. The operating principle of these servos is simple, the servo compares it current position with the input Pulse Width Modulated (PWM) signal and will adjust itself to reach that position. These servos are extensively used in mobile robot applications because of its size and availability.

\subsubsection{Actuator selection}

The actuator selection plays major role in deciding the size of the FRMC prototype. So the actuator selected should have all the important features like less speed, high torque, compact in size, inexpensive, readily available and easy to control. In the above mentioned all the motors RC servos will be suitable for our project because of its small size, inexpensive and easily available. $\mathrm{RC}$ servo has a built-in potentiometer to measure the shaft position of the servo. This servo compares the position of the motor with the input PMW signal and moves its position until that matches the input PWM signal. The pulse repeats every 14 to $20 \mathrm{~ms}$ (milliseconds). If the pulse width lasts for approximately $0.6 \mathrm{~ms}$, the servo will rotate to a maximum position. If the pulse width is increased to approximately $2.4 \mathrm{~ms}$, the servo will rotate to the opposite maximum position. A $1.5 \mathrm{~ms}$ pulse will set the servo in the middle (neutral) position [16, 27]. Fig 3.3 and fig 3.4 represents the servo timing diagram and sectional view of the futaba servo motor. 


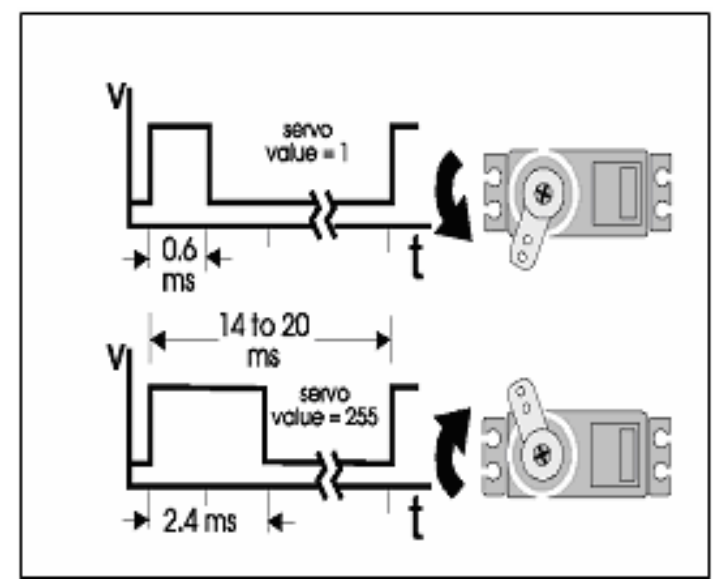

Figure 3.3 Servo timing diagram

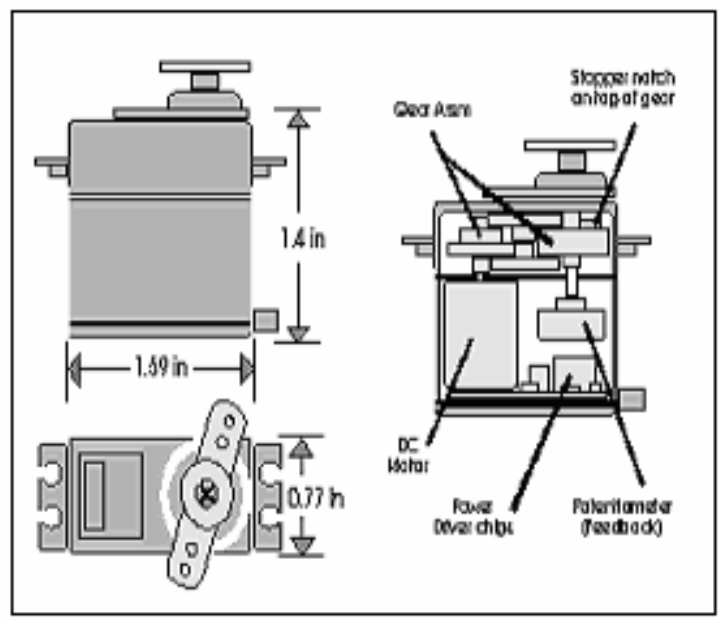

Figure 3.4 Futaba servo motor

These RC servos are used in model airplanes and cars, but when used with the servo controller SV203, it can be made to perform in various systems such as joysticks, minirobotics, animatronics, and computer motion control. These RC servos are designed in such a way that, it turns only 90 degrees of angle in either direction, which comes to 180 degrees of motion. In order to make the servos to use in a situation that require 
continuous rotation they have to be hacked. This modification is done by de-coupling the feedback potentiometer from the output gear and set at constant 90 degrees mark. Thus, when the signal less than 90 degrees is given to the motor, the motor turns at full speed in one direction and vice versa. Since the feedback is removed the servo will continue to rotate as long as the signal remains. The properties of Futaba RC servo selected for the design of FRMC are shown in table 3.1.

Table 3.1 Listing of specifications for futaba electric gear motor

\begin{tabular}{|c|c|c|}
\hline \multicolumn{2}{|c|}{ Futaba electric motor model number: S3004 [13] } \\
\hline $\begin{array}{c}\text { Motor Model } \\
\text { Specifications }\end{array}$ & Units & Values \\
\hline Output Torque & Oz-in & 56.9 \\
\hline Maximum Speed & Rpm & 53 \\
\hline Size & in & $1.59 \times 0.78 \times 1.42$ \\
\hline Weight & Grams & 38 \\
\hline Cost Estimate & $\$$ & 15 \\
\hline Operating Temperatures & Celsius & 0 to 40 \\
\hline
\end{tabular}

\subsection{Sensors}

Measurement of robot parameters is very essential for a successful industrial robot. Today, most of the robots use a component called sensor which is one of the important component of the robot for its survival. Sensor is defined as a device that converts some physical quantity into electrical quantity. A typical sensor consists of a transducer and an 
electronic circuit. In robotics, depending on their characteristics these sensors are divided into two categories; internal sensors and external sensors. Internal sensors are those sensors which are used to measure the robot parameters relative to the reference frame of the robot such as wrist force, joint angle, force, position and mass etc., while the external sensors are those sensors which are used to measure the environment properties. Some of the internal and external sensors used in today's robotics field are discussed below [2]

3.2.1 Proximity sensors: Sensor that detects whether an obstacle or object is close to it without touching it. Optical proximity sensors have a light emitter and receiver through which they sense whether the object is within the sensitive range. If the object is within the sensitive region the light beam after emitting is received back otherwise the light beam is not collected by the receiver. These sensors will work in the range of 0 and $500 \mathrm{~mm}$ with beam angle of 10 degrees. Some of the proximity sensors also use electro magnetic induction. Inductive proximity sensors are used for a range of 1 to $20 \mathrm{~mm}$. These sensors also use a technique of modifying the signal in case of air flow and fibre optic sensors. Airflow sensors use the flow of air to detect the object. If the object is present the air flow is blocked causing back pressure near the orifice from where air is injected. Magnetic field sensors detect the changes in the magnetic field. These proximity sensors are best suited for environments where the robot and object should not touch each other by preventing collisions and damages to the robot.

3.2.2 Range sensors: Sensor that detects an object at a distance called range or depth. These sensors use two physical principles: the time of flight of a pulse and 
triangulation. This time of flight sensors emit a pulse and will measure time between pulse emitted and received. Here both transmitter and receiver lie on the same axis. To calculate the distance of the object we use

Distance $=($ Time of flight $\mathrm{x}$ Speed in medium $) / 2$

Radar is the most common example of the time of flight pulse. Ultrasonic range sensors are used to find the distance of the obstacle. Time of flight measurements over short distances is feasible with ultrasonics as speed of sound is much slower than light. Triangulation sensor uses the geometry to find the distance of the object, which requires detection of the object from two different view points. When the light reflected from the object is detected, the emitter and detector angles are recorded, and the distance or range is calculated from those angles through geometry. Laser sensors use a laser diode as a light source, and a linear photo diode as a detector. From the angle of reflection the distance of the object is calculated. This sensor is more effective when the object is in the range of 5 to $60 \mathrm{~mm}$.

3.2.3 Touch sensors: By using touch sensors we can find two characteristics of the object: its presence and its shape. Most tactile sensors uses switch to sense the object and its shape. A whisker sensor emulates a human hair and detects the object before it collides. The whisker will detect the object by touching the object but this will not damage the robot as it is flexible. When compared to vision sensor, touch sensor provide data which is directly related to the variable being measured; shape position, orientation. 
3.2.4 Force/Torque sensors: The force/torque sensor is particularly suitable for applications requiring simultaneous measurement of several forces and moments, or measurements of forces that change direction and position over time. Common applications for this sensor include research and development in robotics, production processes, biomechanics, and dynamics. A waterproof version is available for use in tow tanks, ocean engineering, and other underwater applications.

3.2.5 Potentiometer: A Rotary potentiometer is an instrument which is used to measure the position of the shaft when attached to motors by measuring the difference of electric potential between the two points. Linear potentiometers are common contact transducers in the form of variable resistors with three leads. Two leads connect to the ends of the resistor, so the resistance between them is fixed. The third lead connects to a slider or wiper that travels along the resistor and the resistance between it and each of the other two connections varies.

3.2.6 Vision sensors: Those sensors which see and recognize the object by collecting the light reflected by the object into an image and processing that image. Some electronic devices or computers will process the data collected and will analyze the image of the object. By using these sensors the robot and the object will not touch each other, and are operated with some distance apart; which makes it to be used in hazardous environments where human cannot perform the task. In teleoperation, these vision sensors play very important role. Cameras can be used as the vision sensors in teleoperation and still research is being conducted on what type of vision sensors are to 
be used for teleoperation to solve the vision related problems. Most of the image processing by these vision sensors requires complex algorithms that need to be run on the microchip at real time.

3.2.7 Tachometers: Tachometers are used for measuring the speed of the motor shaft. These are classified into two categories: contact type tachometers and non-contact type tachometers. Contact type tachometers are those which require contact with the surface whose speed has to be determined. Non-contact type tachometers are those which do not require contact with the surface whose speed has to be determined instead the speed is determined by focusing a beam of light on the surface. Laser tachometers are the most commonly used non-contact type tachometers.

\subsubsection{Sensor selection}

The sensor which is readily available, compact, inexpensive and effective should be chosen for our project. Keeping all these factors in mind we have selected to use Sharp GP2D12 analog infrared ranger $[13,17]$. These sharp IR rangers use triangulation technique and small linear CCD array to determine the distance of the object from the robot. The sensors which use triangulation techniques are much more efficient than the sensors which use time of flight technique. This sensor has connector with 3 pins; power, ground and the output voltage. This sensor works effectively when the object is within a range of $10 \mathrm{~cm}$ to $80 \mathrm{~cm}$. The operating supply voltage is between 4.5 to 5 volts. As shown from the graph below, the output voltage that is generated from the sensor represents a value of a trigonometric function, because of this, the output of these sensors 
is non-linear with respect to the distance being measured as shown in Fig 3.5. Fig 3.6 shows the GP2D12 sensor [27].

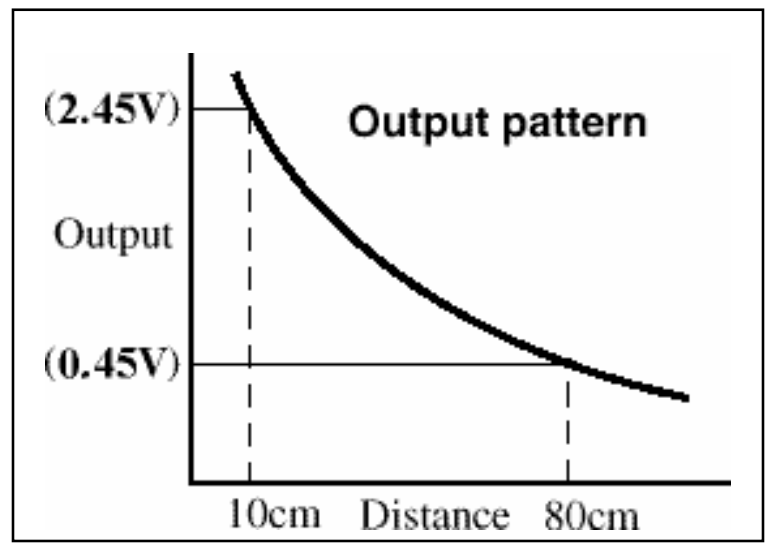

Figure 3.5 Non-linear graph

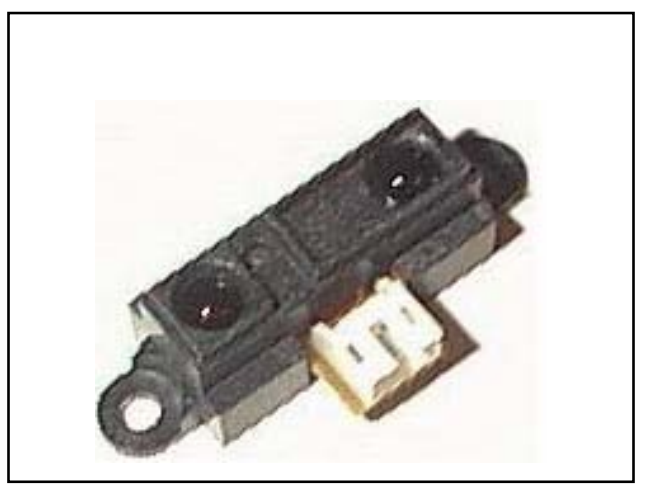

Figure 3.6 GP2D12 sensor

In case of no object, the light emitted is never reflected back. If the light emitted is received by the detector then a triangle is formed between the point of reflection, the emitter and the detector, which is shown in fig 3.7 below. From that triangle the angle of reflection is calculated and thereby the distance of the object. 


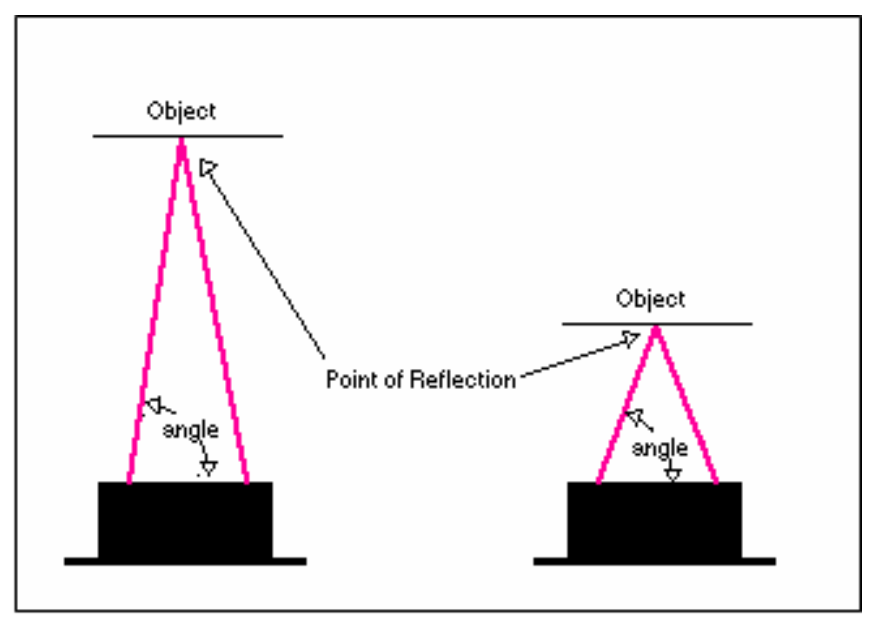

Figure 3.7 Distance through triangulation

The internal block diagram of the SHARP GP2D12 IR sensor is as shown in the fig 3.8 below:

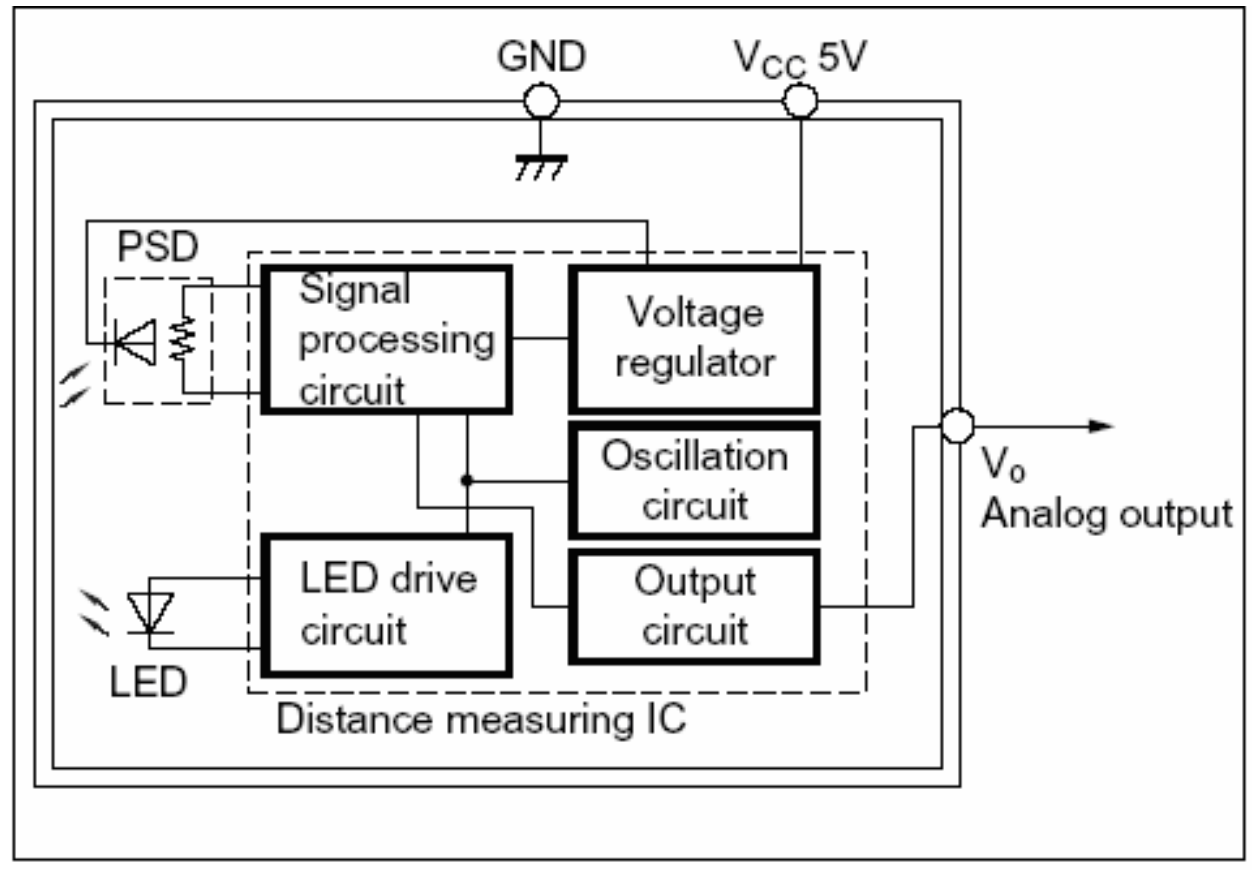

Figure 3.8 Internal block diagram 


\section{CHAPTER 4}

\subsection{MICROCONTROLLERS}

Microcontrollers are considered to be the heart of a system wherever it is used. A microcontroller usually consists of microcontroller chip, voltage regulator, memory and input / output ports. There are various microcontroller producing companies in the market. When designing a small manual controller prototype (1-DOF FRMC), we need to consider the shape, size, cost, availability and its functions before deciding which microcontroller should be used. Every microcontroller has to be programmed to use it for some application.

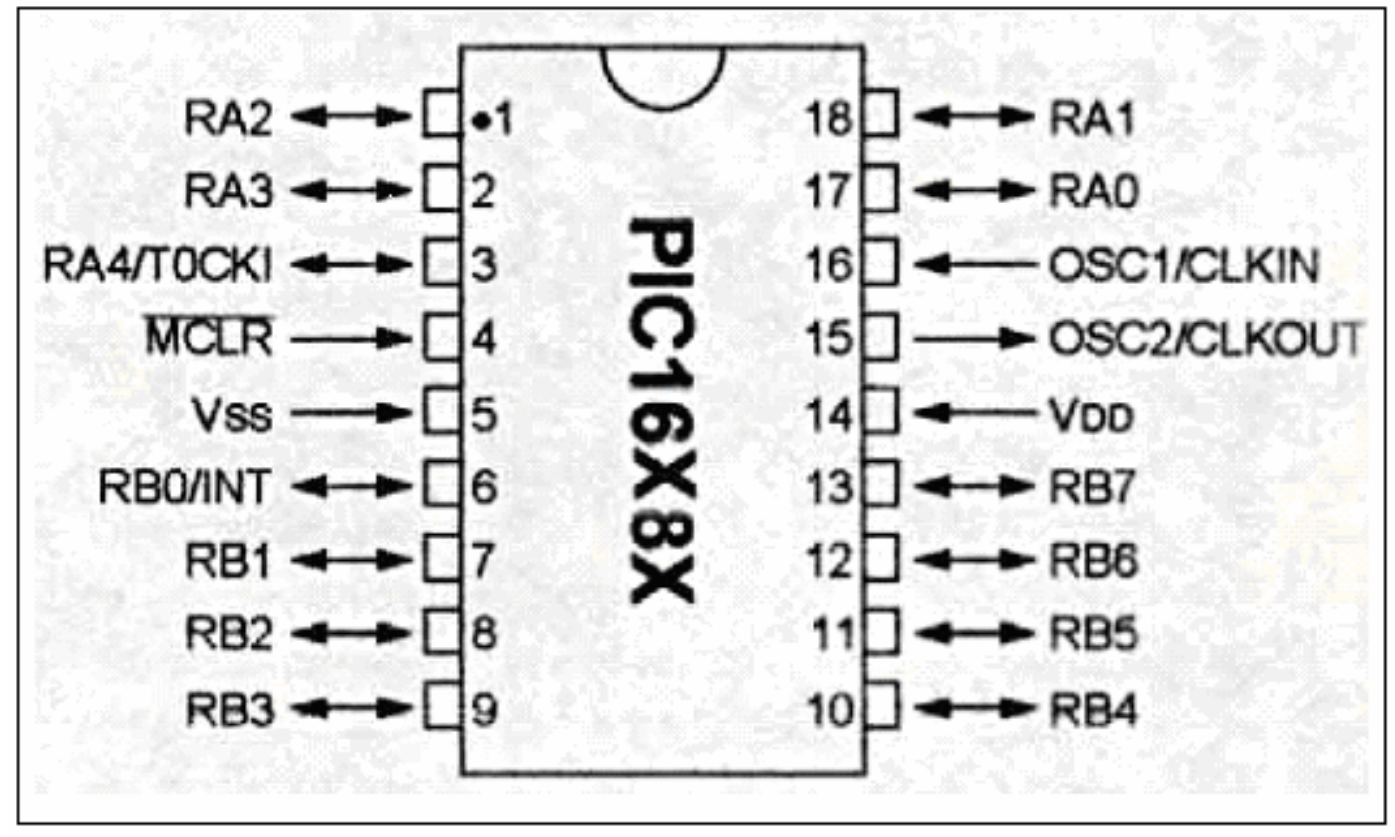

Figure 4.1 PIC microcontroller

In robotic applications, based on the robot's input, the internal programming in the microcontroller can command the motors in such a way that the robot works efficiently. 
Of all the microcontrollers Programmable Integrated circuits (PIC), are the simplest microcontroller chips produced by Microchip Technology. Fig 4.1 shows the PIC microcontroller.

This PIC microcontroller should be programmed to be used and it can be programmed by using Basic, $\mathrm{C}$ or Assembly language. Some of the microcontrollers and boards that are being used in the robotic applications are described below.

\subsection{Mini board:}

The Mini Board was developed at Massachusetts Institute of Technology (MIT) for a robot course and design project. It is a small and inexpensive design for a controller board which uses $68 \mathrm{HC} 11$ micro-controller. The MINI BOARD 2.1 is a complete embedded computer board for robotic applications. A typical Mini board is shown in fig 4.2 below [25]. It can directly supply power to four DC motors and receive inputs from many sensors. Its small size makes it suited well for mobile applications as well as other embedded control. It can be programmed in 6811 assembler code or $\mathrm{C}$ for stand-alone operation, or it can serve as a serial-line based controller operated by a desktop computer. The Mini Board 2.1 Extended is the latest version of the Mini Board 2.0. Mini Board uses Motorola $68 \mathrm{hc} 811 \mathrm{e} 2$ microprocessor with 2048 bytes of internal, EE PROM and 256 bytes of RAM which stores approximately 2000 instructions. This board can control four DC motors bidirectional at voltages 5.6 to 36 volts and up to $600 \mathrm{~mA}$ current. It has eight analog inputs, eight digital inputs or outputs, several timer and counter I/O pins. It uses RS-232 compatible RJ-11 port for communication/program download between host computers. 


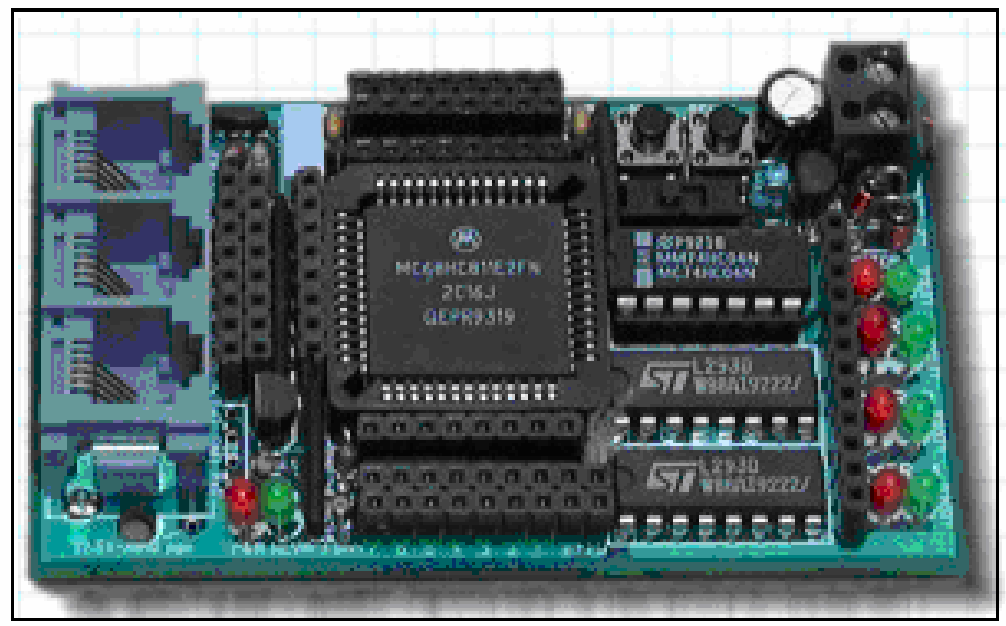

Figure 4.2 Mini board

\subsection{Handy board:}

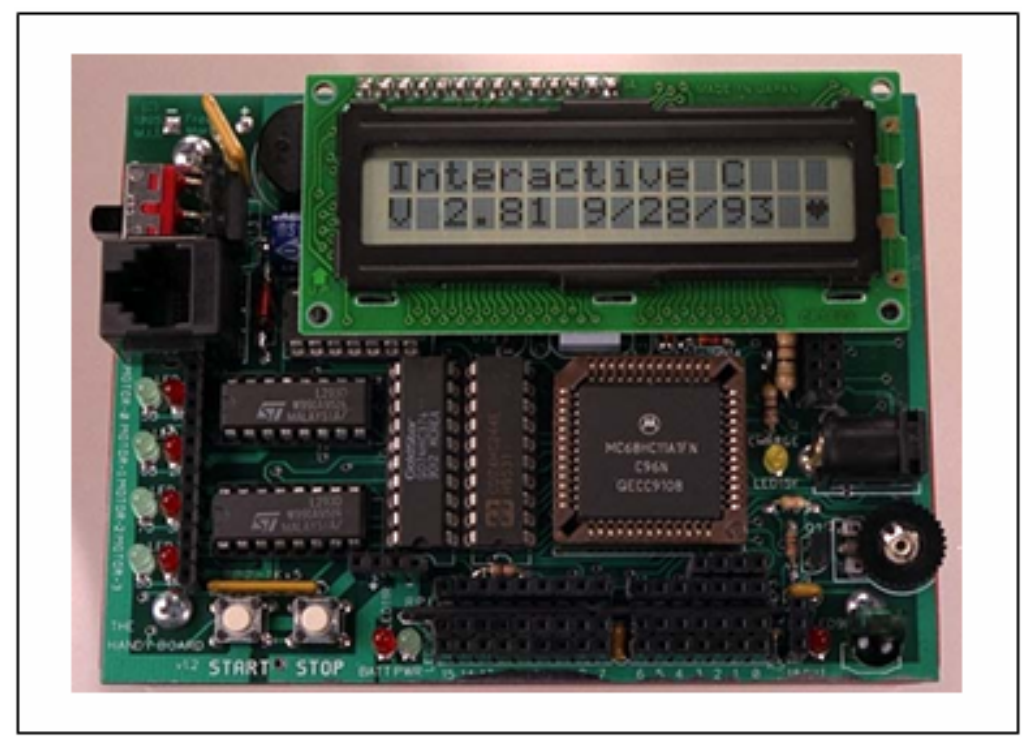

Figure 4.3 Handy board

A new microcontroller board ideal for experimental and educational robotic projects called handy board was developed by Fred Martin at MIT shown in fig $4.3[24,28]$. This handy board is the extended version of the mini board. Some of the important features of 
this handy board are; Small board size (4.25 x 3.15 in), uses 52 pin Motorola 6811 microprocessor at $2 \mathrm{MHz}$, has $16 \times 2$ character LCD screen, has inputs for 7 analog and 9 digital sensors, has $38 \mathrm{KHz}$ IR receiver and transmitter, connection to the desktop compute COM port (Rs-232), requires separate serial interface board. Since it is used in educational institutions, the software used should be readily available, so interactive $\mathrm{C}$ is commonly used to program these handy boards. Its small size and software choices make it used for commercial purposes.

\subsection{Bot board:}

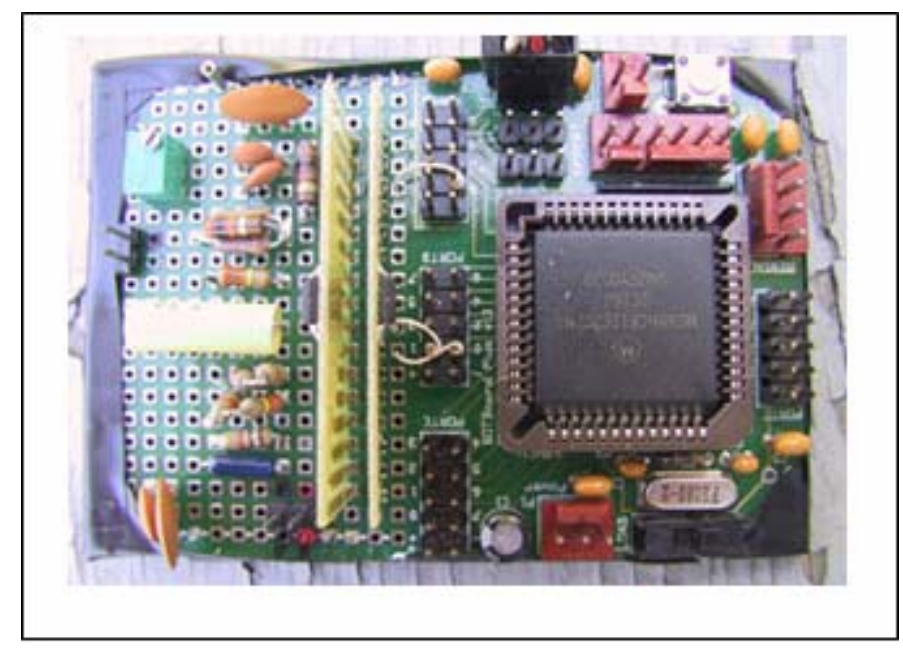

Figure 4.4 Bot board

Bot board uses popular $68 \mathrm{HC} 11$ microcontroller with a minimum configuration designed for general purpose and also for the robotic applications. Some of the features of this Bot board are; it has a small size of $5 \times 7.5 \mathrm{~cm}$, is capable of controlling up to $4 \mathrm{R} / \mathrm{C}$ servos, powered with RS-232 for serial port communication, has multiple input / output ports, gives more flexibility for least cost. Fig 4.4 shows the generally used Bot board. 


\subsection{MTJPro11 microcontroller:}

This microcontroller is one of the best $68 \mathrm{HC} 11$ microcontrollers developed by Mekatronics shown in fig 4.5 below. It is very small in size and is very inexpensive when compared with other microcontrollers with same features. Some of the important features of this microcontroller are; can control up to 5 servo motors, has $32 \mathrm{~KB}$ of memory, has a serial communication interface, it has 8 analog input channels and 3 digital input / input capture, it has output of $40 \mathrm{KHz}$ clock signal to module IR \&sonar, 8 digital output pins. The only main disadvantage when compared to other microcontrollers is that it does not have an LCD screen [28].

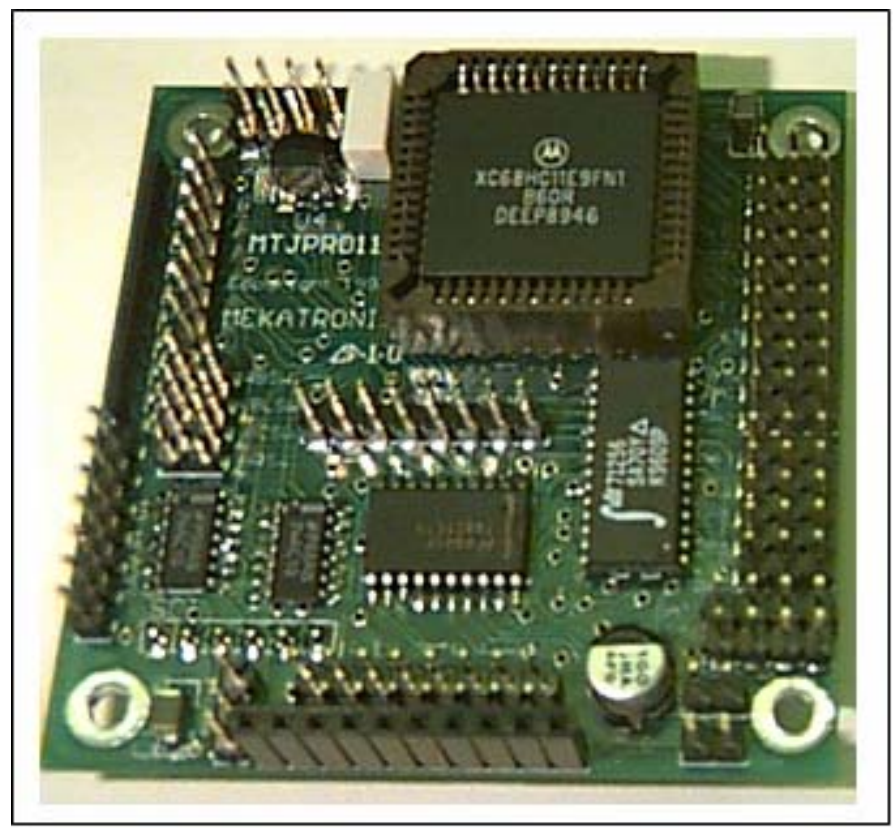

Figure 4.5 MTJPro11 microcontroller 


\subsection{Basic stamp:}

Basic Stamp is a small microcontroller where a PIC microcontroller is embedded inside its design, and is developed by Parallax. This microcontroller with its operating system embedded within requires only a program to run it. The language used to run this microcontroller is the Basic language. Fig 4.6 below shows Basic stamp 2 [23].

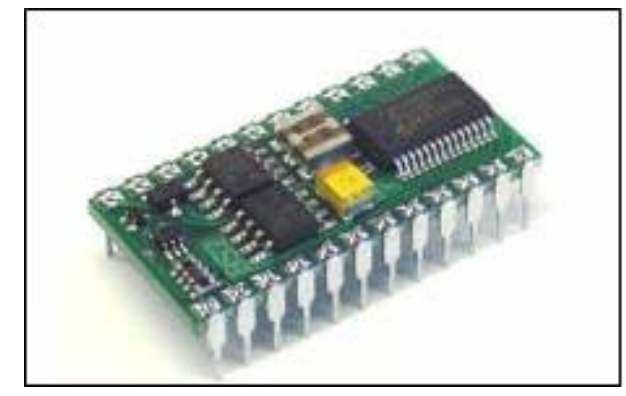

Figure 4.6 Basic stamp

Some of the important features of the Basic stamp include an ability to count cycles on a pin; 16 input / output lines, which are usually used to connect to LED's, potentiometers, push buttons, shift generators and speakers; generating PWM signals and sine waves; PBASIC interpreter; non-volatile EEPROM; resonator; and a 5-volt regulator.

When 5-to-15 volts are applied, the interpreter reads and executes the PBASIC instructions from the EEPROM. BASIC stamps are used in educational institutions, industrial applications, and hobbyist robotics projects. Usually BASIC stamps are able to execute up to 10,000 interpreted PBASIC instructions per second. 


\subsection{Other microcontrollers:}

The JS2051A1 CPU BOARD is a very small CPU board that uses an ATMEL AT89C2051 CPU. The AT89C2051 is an INTEL 8051 CPU compatible microcontroller. It has an extra analog comparator and internal $2 \mathrm{~K}$-byte flash memory. Some of the features of this controller are listed as its small size of $36.8 \times 20 \mathrm{~mm}$, use of an LCD display and a 5 V DC supply. Fig 4.7 shows a typical AT89C2051 microcontroller below [24].

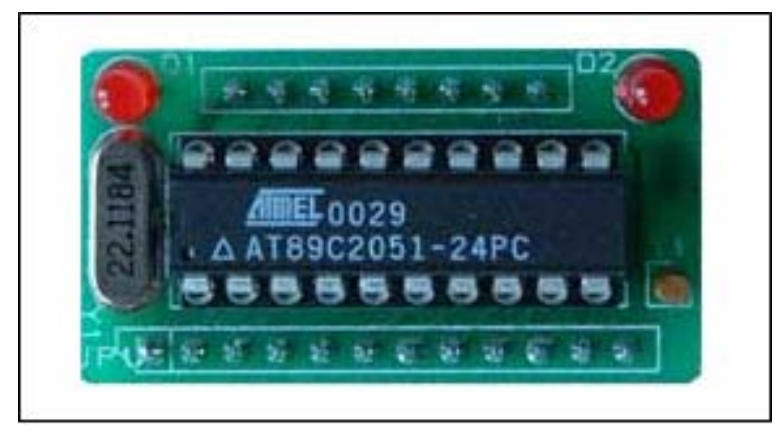

Figure 4.7 AT89C2051 microcontroller

The OOBoard is based on the OOPIC-R processor. The OOBoard with the OOPIC-R can be programmed by using any of the computer languages such as C, Java, Basic, or an object-orientated language. The OOBoard shown in fig 4.8 has the following features such as dual power supply connectors, dual operating modes selectable by a switch, three user-controllable switches, three user-controllable push buttons, a speaker, a serial LCD connector, TTL serial connector, RS232 serial port, three servo connectors and an hbridge connector [24]. In order to power the board, two regulators are installed: UltraLow Drop-Out regulator for the microprocessor, and a $5 \mathrm{~A}-5 \mathrm{~V}$ accessory regulator for 
motors, sensors, etc. The board also contains a constant current $(\mathrm{C} / 10)$ battery charger for NiCad packs.

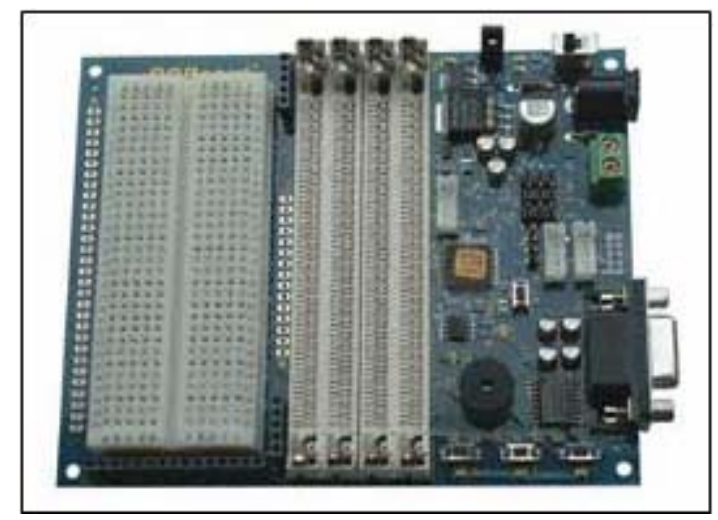

Figure 4.8 OOBOARD

\subsection{Servo controllers:}

Servo controller boards have been designed to control servo motors. In general, they can drive more servomotors than a single microcontroller can. Microcontrollers have an advantage of input options for sensors; whereas some servo controllers don't have this feature. Our project mainly deals with controlling servos so that we will be able to make a study on servo controllers as well. Some of the commonly used servo controllers in the robotic applications are discussed below.

\subsubsection{Brainstem controller:}

This BrainStem Module offers 2 channels of high-resolution motion control. These channels offer flexible PWM or PID control of motors with various types of feedback including encoders, quadrature encoders, analog input, and Back-EMF speed control. The 
main features of this controller are, it has; $40 \mathrm{MHz}$ RISC processor, one $10 \mathrm{bit} \mathrm{A} / \mathrm{D}$, status LED, 368 bytes of RAM, RS232 serial port, small size ( $2.5 \times 2.5$ in). The Brainstem controller is as shown in the fig 4.9 below [26].

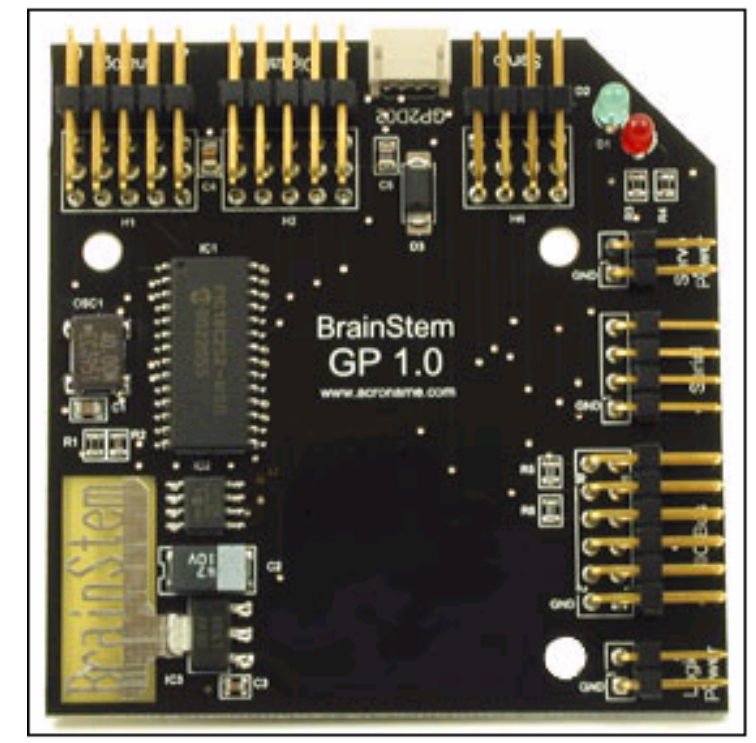

Figure 4.9 Brainstem controller

\subsubsection{Pololu servo controller:}

The Pololu servo controller shown in fig 4.10 can control up to $8 \mathrm{R} / \mathrm{C}$ servos [22]. This Pololu servo controller comes in two different configurations, where one can control a maximum of eight $\mathrm{R} / \mathrm{C}$ servos and the other controls up to sixteen $\mathrm{R} / \mathrm{C}$ servos. The interface with the computer to the servo controller is usually through an RS232 serial port or a TTL serial line at baud rates (maximum number of bits of information, including control bits, that are transmitted per second) of 1200 to 38400 baud. Some of the features of this servo controller include its small size of $1.45 \times 1.7$ in, eight servo ports, and a 
power supply with a range from 5.6 to $25 \mathrm{~V}$. The only disadvantage of this servo controller is that it does not have a port to connect any sensors.

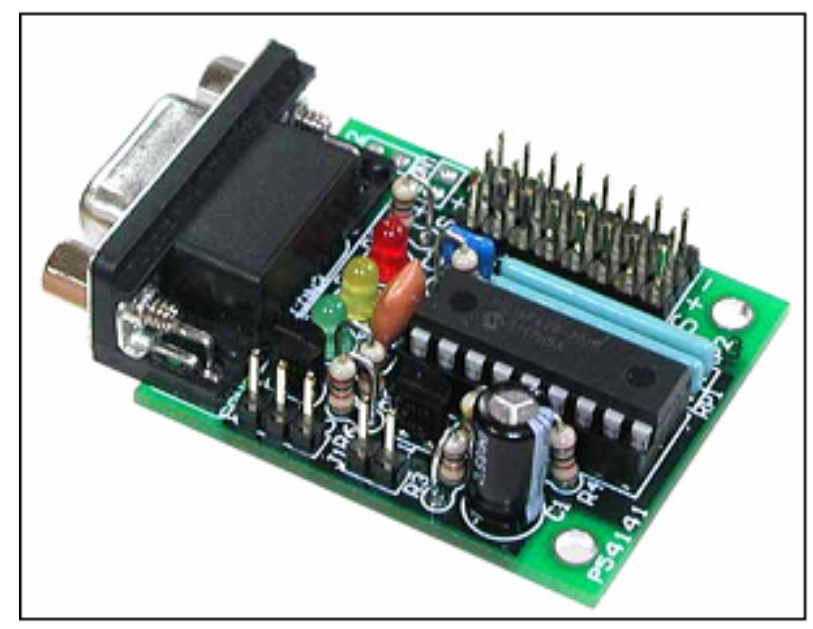

Figure 4.10 Pololu servo controller

\subsubsection{USB servo controller:}

USB Servo controller was developed by Lynxmotion to control R/C servos using the USB port attached to a computer. Fig 4.11 below shows how USB servo controller looks like [21]. This servo control can control up to eight servos.

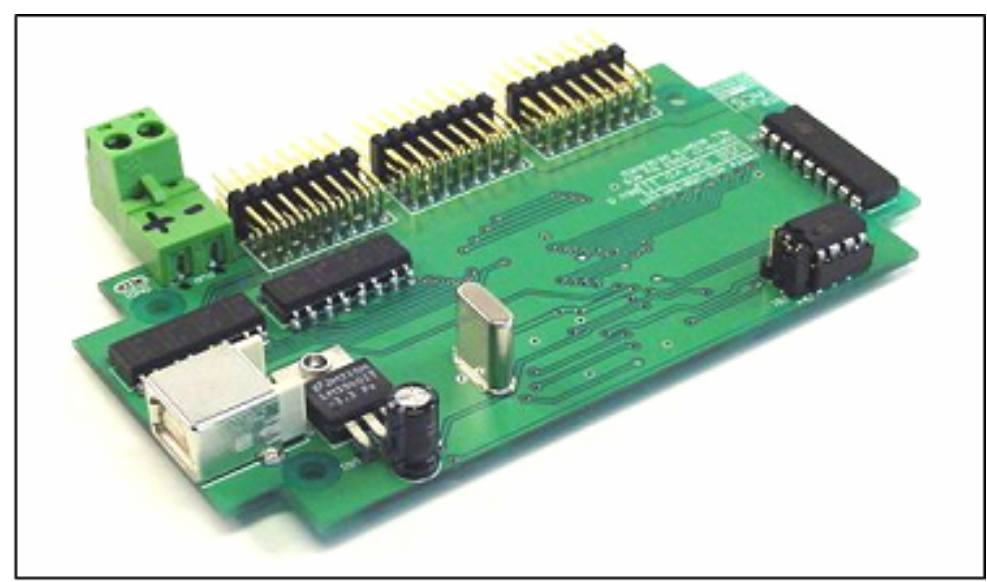

Figure 4.11 USB servo controller 
It has eight optically-isolated inputs and also outputs. Power to the servo motors has to be supplied form the external power source. Lynxmotion has also developed two other types of servo controllers. Absence of sensor input is the main disadvantage in all the three servo controllers developed by the Lynxmotion.

\subsubsection{Pontech SV203 servo controller:}

SV203 Servo controller is used to control R/C servos when connected to a computer through RS232 serial port. This SV203 servo controller was developed by Pontech and can control up to eight servo motors. It has a PIC16C73 microchip embedded in it. This controller accepts serial data from an RS232 port and outputs PWM signal to control R/C servos. Unused servo pins can be reconfigured to digital output in order to drive on/off devices. Fig 4.12 shows the SV203 controller [28].

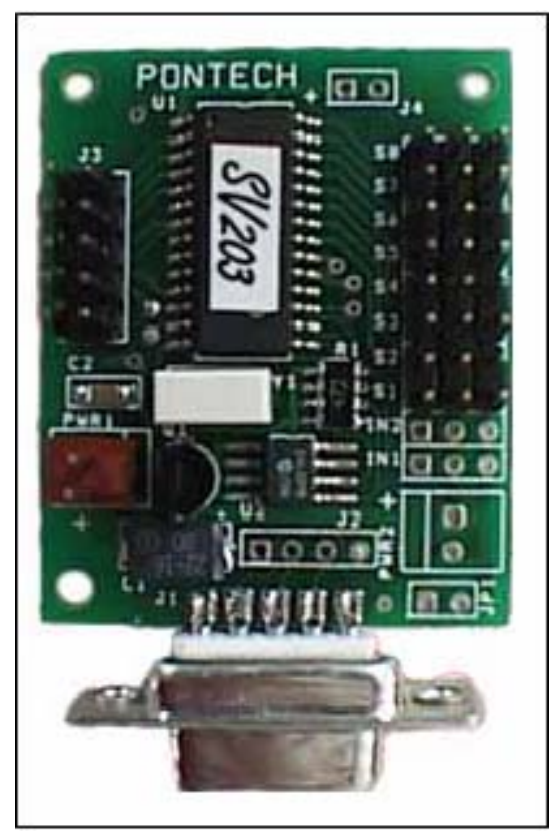

Figure 4.12 SV203 controller 
This servo controller has eight $\mathrm{R} / \mathrm{C}$ servo inputs and five $\mathrm{A} / \mathrm{D}$ inputs. The board requires a power supply of 7-15 $\mathrm{V}$ and the $\mathrm{A} / \mathrm{D}$ input power supply has a range of 0 -to-5 $\mathrm{V}$. This servo controller is very small in size compared to other controllers (1.4 x 1.7 in). The SV203B/C has the added feature of being able to run a standalone BASIC program on board through an 8K EEPROM (Electrically Erasable Programmable Read Only Memory). An optional IR (Infra Red) Receiver/Transmitter (IR 100) is also available to allow infrared serial communications. A 5-channel, 8-bit A/D input is available to read analog voltages between 0 and 5 Volts. Devices such as analog joysticks or potentiometers can be connected to this port and the position can be read by the PC and sent back to the board to control the servo position 


\section{CHAPTER 5}

\subsection{FRMC AND PLATFORM DESIGN}

\subsection{Force-reflecting manual controller system design}

In the design of FRMC the motor selection, controller selection and the type of transmission system used are important criteria to be considered while designing the system. With this 1-DOF FRMC design, any one-degree-of-freedom system can be controlled; therefore, the research results are expected to be broader than a 1-DOF system might imply. In order to design the 1-DOF FRMC described above, six factors should be considered: (1) to accomplish a very low weight in the device, (2) to have a compact robotic system, i.e., overall size the robot should be small, (3) to transmit the power to the haptic device with very low power loss (torque), (4) to select a suitable motor and a controller, (5) to program the robot in an efficient way, and (6) to assemble and demonstrate the final performance of the entire system. The design parameters also include selection of a potentiometer, selection of the sensor, and developing the necessary software programs to accomplish remote control with the force reflection feature. The process of design will be carried out by using popular engineering software packages; namely, Pro/ENGINEER drawing tool, and Visual Basic programming tool. Performance of the system will also be demonstrated as part of this work.

Among the developed 1-DOF FRMC concepts, we have chosen the direct drive mechanism, which is judged to be more efficient than others. We believe that we can satisfy the goal of a compact design with a 1-DOF device whose size and motion are similar to that of a PC mouse. Since fingers will manipulate the device, it should be 
capable of producing a maximum force reflection of about 2 to $4 \mathrm{lb}$. In this concept, the joystick is directly attached to the actuator's shaft. The direct drive mechanism is as shown in fig 5.1.

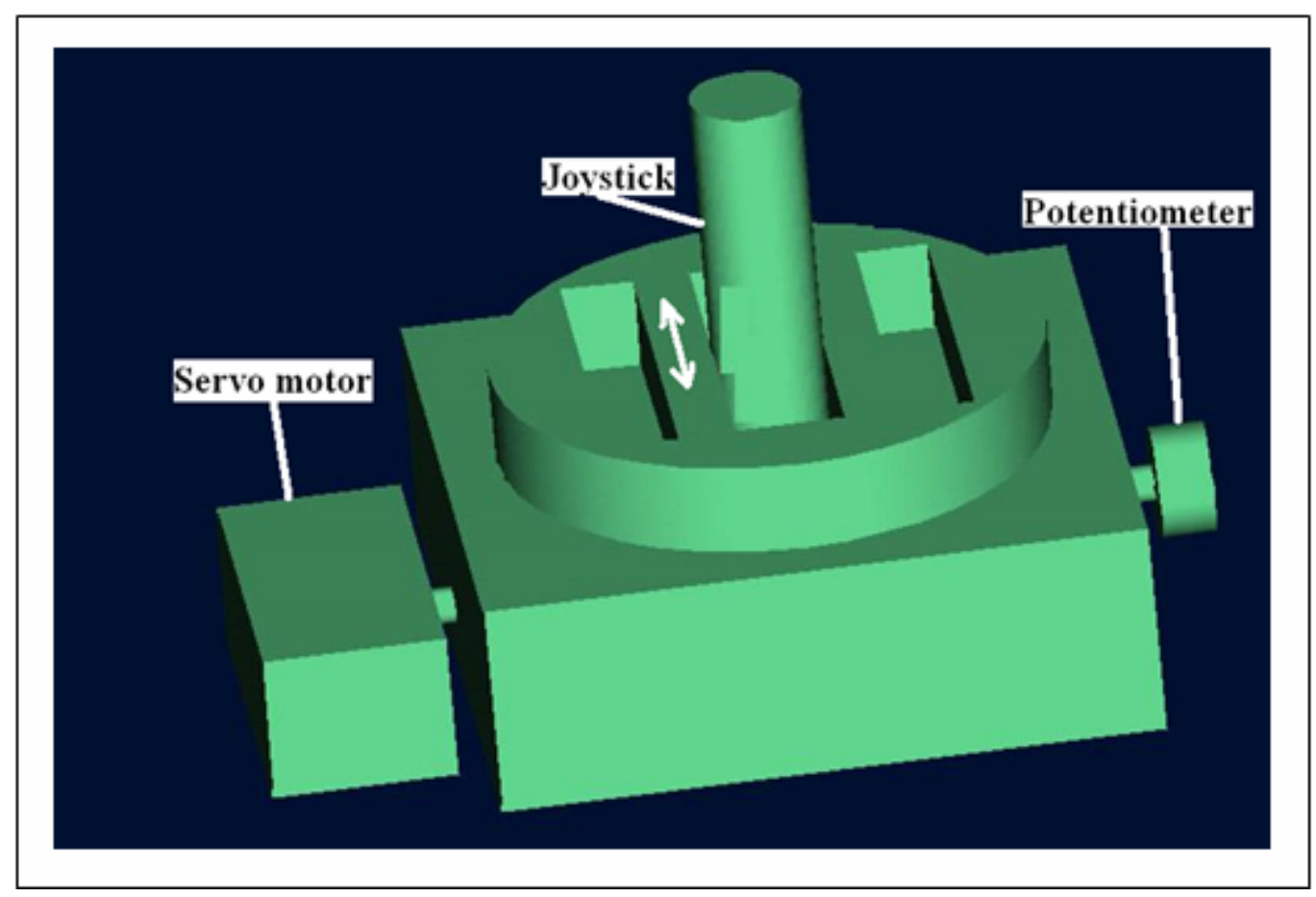

Figure 5.1 Direct drive mechanism

By using this concept of direct drive method in FRMC makes the design more compact and also more efficient as it directly couples to the joystick. The maintenance of this FRMC is inexpensive and the total cost for building it is also less costly compared to the other methods. By directly coupling the motor shaft to the joystick, we can have acceptably high torque and also smooth operation. Due to the direct linking of joystick to the motor shaft we don't have any intermediate losses. We also don't have any frictional losses or backlashes in this case. However, unlike the gear transmission and belt 
transmission systems, a direct drive system doesn't reduce speed and also there is no torque amplification.

Finally the manual controller was made from the following components:

- Microcontroller board

- Servo motor

- Power supply

- Infrared sensor

- Potentiometer

We have selected SV203 servo motor controller which uses PIC16C73 microchip developed by Pontech as the microcontroller for our case, and the advantages of using this micro controller in our case is described in chapter 4. We have selected Futaba S3004 as the required servo motor and GP2D12 IR as the required infrared sensor for our design. The selection of these two parameters is described in chapter 3 . The connection of the servo motor with the SV203 board is as shown in fig 5.2 below.

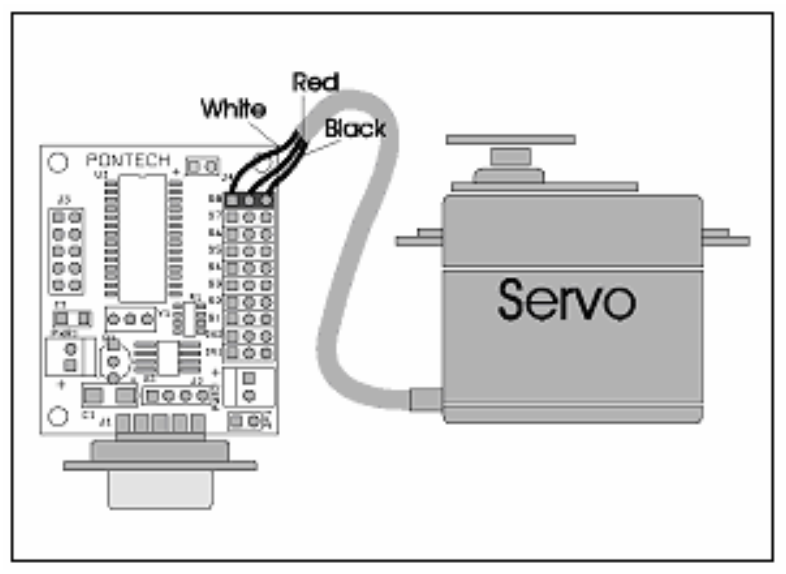

Figure 5.2 Connection of servo with SV203 


\subsection{Power supply and pin-out}

A 6-Volt DC source powers the SV203 micro controller board, either from 4 alkaline batteries or $5 \mathrm{NiCad}$ (Nickel Cadmium) cells. An AC adapter can also be used: 6VDC, at 300mA. If using NiCad, a 4-cell pack might be easier to find than a 5-cell pack. The board will operate fine with 4 cells, but may not last as long as 5 cells can. Given below in Figure 5.3 is the pin out diagram, which explains in detail the entire circuit board terminals and the respective pin outs.

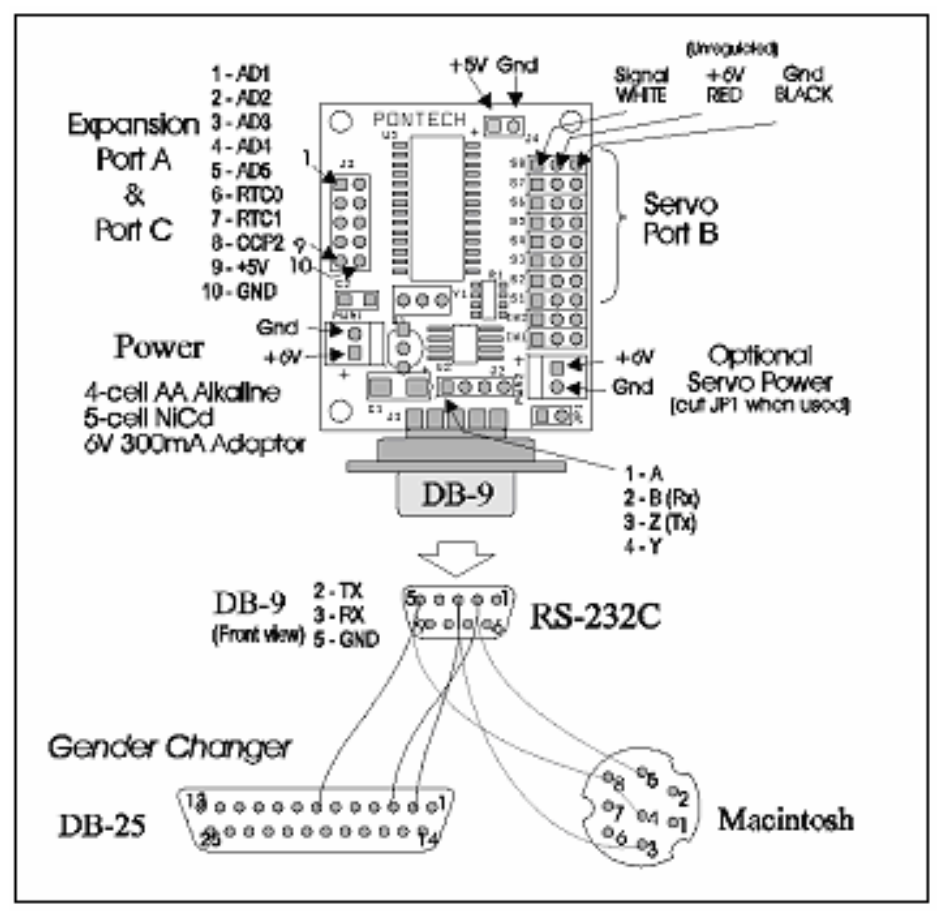

Figure 5.3 Pin out diagram

The potentiometer is used to measure the shaft position of the servomotor and sends it to the compute through SV203 board. The potentiometer is attached to the servomotor as shown in the fig 5.4 below. 


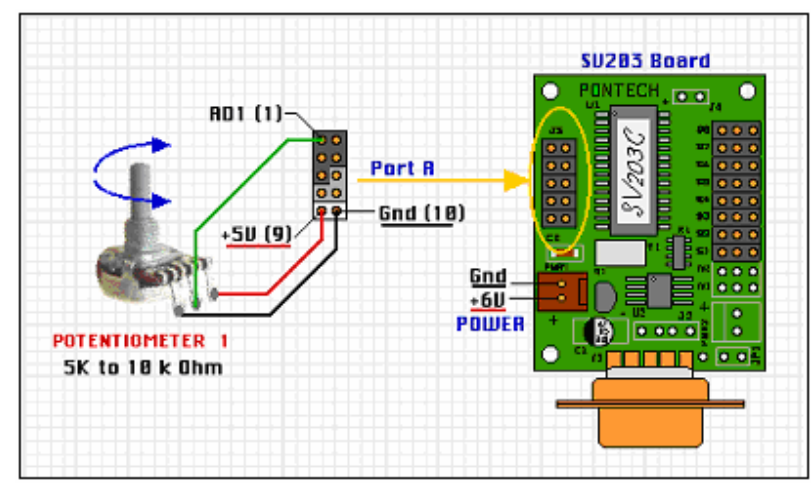

Figure 5.4 Connection of potentiometer with SV203

\subsection{Housing}

The main objective is to make the structure as simple as possible and at the same time capable of holding as many as components as possible. The housing structure was made of plexi glass to mount the robot components, along with fastening facilities such as screws and nut housing for mounting the components. Plexi glass was chosen to reduce the overall weight of the robot. The other components are plastic angles for holding and mounting the servo motor and servo controller. Fig 5.5 shows the 1-DOF FRMC developed.

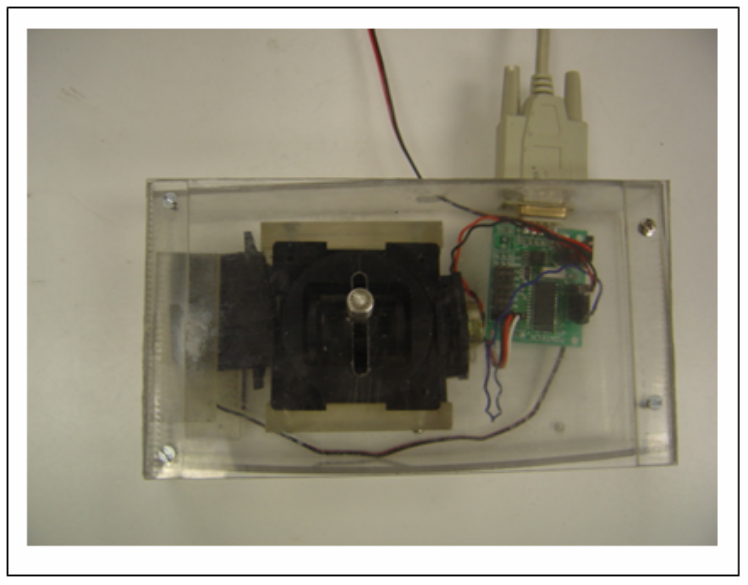

Figure 5.5 1-DOF FRMC developed 


\subsection{Slider bar platform design}

In order to test the 1-DOF FRMC, a platform has to be developed. So initially we have developed a simple 1-DOF sliding bar platform which is built by using a RC servo motor, infrared range sensor, sliding bar, SV203 controller. The system operates at $7 \mathrm{~V}$ of power supply. When the joystick is moved in forward/backward direction, the potentiometer reads the position of the joystick and will send the signal to the SV203 controller, according to this potentiometer feedback SV203 controller produces the signal to activate the slider servo motor to move it in forward/backward direction accordingly. The sensor on one end of the slider bar monitors the path of the moving part and will send the signal to the SV203 controller. Once the moving part (plate) reaches the boundary of the slider bar then the signal from the infrared sensor is sent to the servo controller which in turn moves the servo motor coupled to the joystick to the other side and will produce force reflection if the user still moves the joystick in the same direction and the servo motor which is driving the slider bar will rotate in reverse direction until it reaches the boundary on the other side. Fig 5.6 shows the slider bar platform developed to test 1-DOF FRMC.

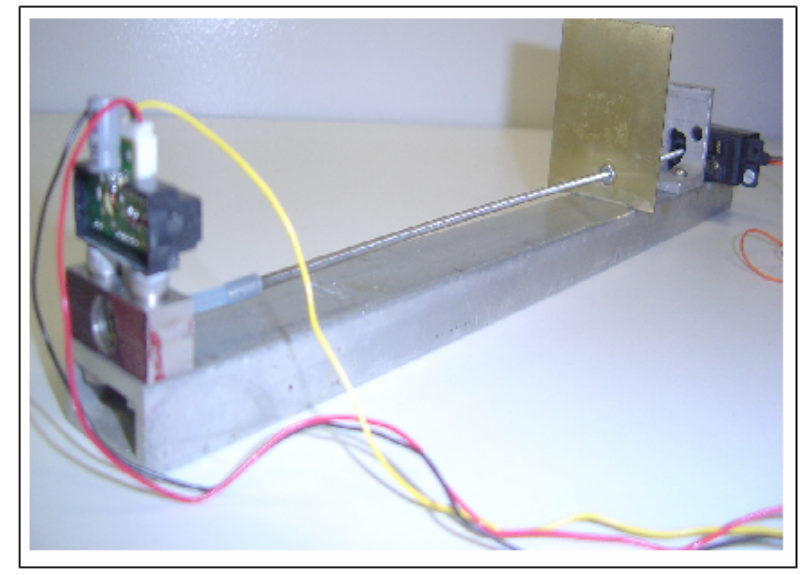

Figure 5.6 Slider bar platform 


\subsection{Palm pilot robot kit (PPRK) platform design}

A platform named palm pilot robot kit was developed by freshman Grigoriy Reshko and Dr. Matt Mason at Carnegie Mellon University (CMU). We have decided to use this PPRK to test our 1-DOF FRMC. Fig 5.7 shows the PPRK designed at CMU. The PPRK is a small robot that uses a palm as brain of the kit. The main purpose of designing PPRK was to make mobile robots available to everyone.

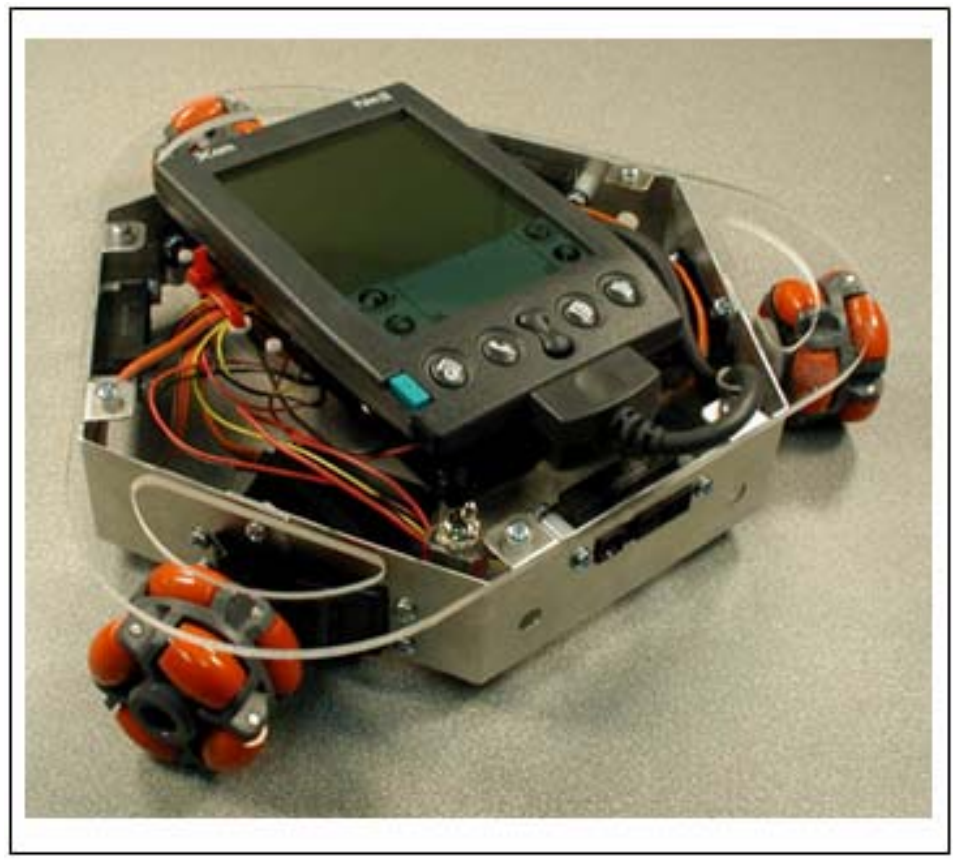

Figure 5.7 PPRK designed at CMU

The PPRK empowers a palm pilot to move about and sense the nearby environment. The base uses three omni-wheels that allow driving in any direction with independent control of rotation, meaning it moves holonomically in the plane. The base also has three optical range sensors to see the nearby environment up to about a meter away [29]. This PPRK uses a Brainstem controller which can be programmed by using Basic, C, C++ or Java. 
The program to control the PPRK is loaded on to the palm pilot and the brainstem controller receives the signal from the palm and responds accordingly. This brainstem controller operates at $6 \mathrm{~V}$ of supply. This kit can also be controlled by the desktop PC instead of palm. The commands are sent to the controller through the RS232 serial port [20]. So we have decided to buy only the required parts and assembled the PPRK platform. Table 5.1 below shows all the required parts to build a PPRK

Table 5.1 parts required to build the PPRK

\begin{tabular}{|l|c|}
\hline \multicolumn{1}{|c|}{ Part name } & Quantity \\
\hline 4cm diameter omni-directional wheels & 3 \\
\hline Modified RC servo motors & 3 \\
\hline Sharp GP2D12 Infrared Rangefinders & 3 \\
\hline Brainstem Controller & 1 \\
\hline Palm Pilot III & 1 \\
\hline 6 V battery & 2 \\
\hline Clear Cast Acrylic Disk & 1 \\
\hline Male DB9 connector & 1 \\
\hline
\end{tabular}

\subsubsection{Palm pilot robot kit (PPRK) platform design modification}

The mobile robot PPRK uses the brainstem controller while the 1-DOF FRMC developed uses the SV203 controller. Integrating PPRK with FRMC may cause a problem while programming. So we have decided to modify the PPRK, by replacing the 
brainstem controller with SV203 controller as it fulfills all the requirements of a brainstem controller. So the modified PPRK will be having SV203 controller instead of brainstem controller, three RC servos, three IR sensors, $6 \mathrm{~V}$ of power supply. The SV203 controller receives the signal from the palm and will send commands to the servos accordingly. The power supply to the sensors is taken from the unused servo pins on the SV203 board as the board cannot supply power through the AD power supply to all the three sensors. Fig 5.8 shows the modified PPRK kit.

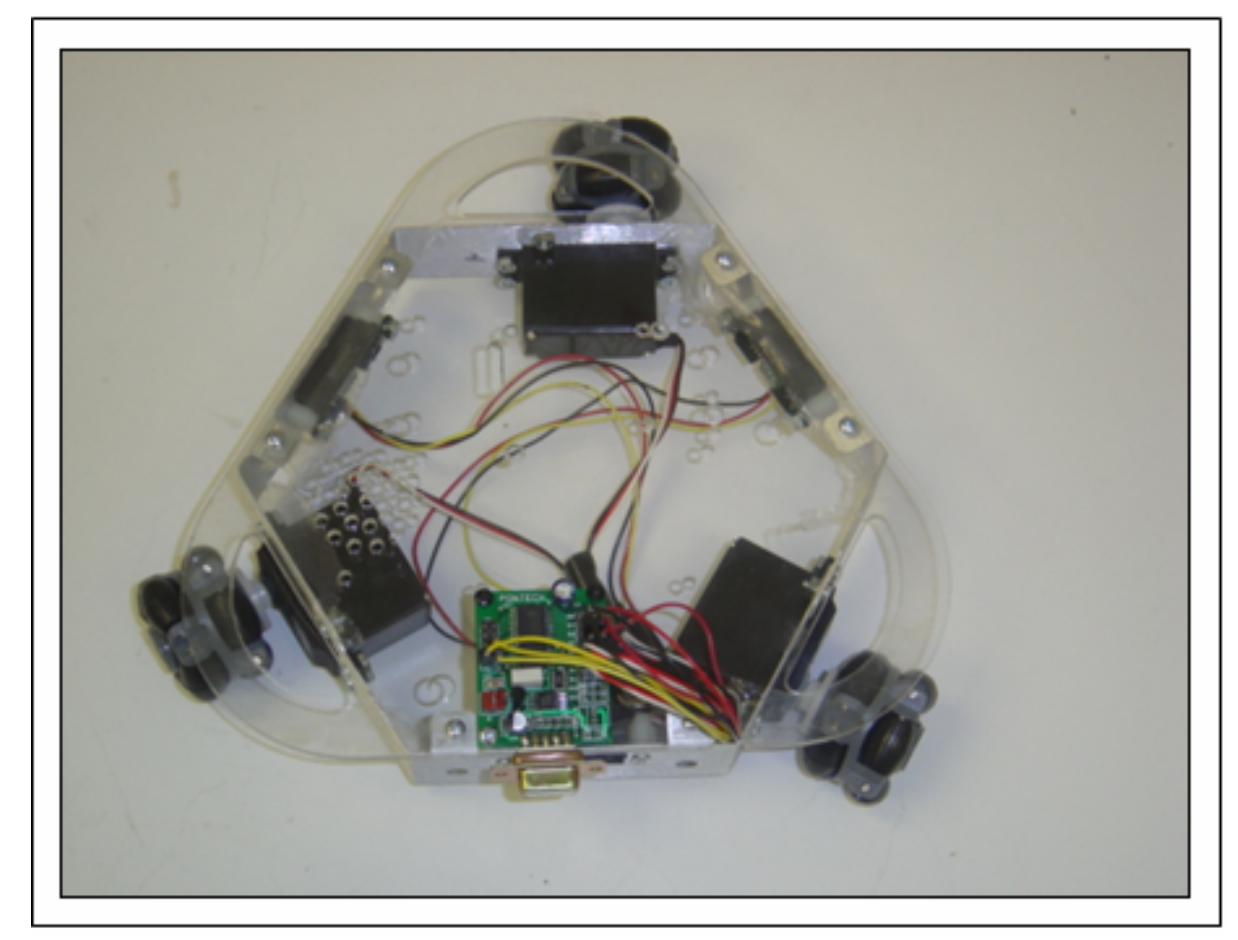

Figure 5.8 PPRK modified mobile platform 


\section{CHAPTER 6}

\subsection{ROBOT CONTROL SOFTWARE DEVELOPMENT AND SYSTEM INTEGRATION}

In this chapter, the software development and system integration of the FRMC controller prototype with the remote system is presented. We initially discuss interfaces developed to control the FRMC step by step, and then demonstrate how the 1-DOF FRMC prototype is used to control remote systems, that include the PPRK platform and the slider bar.

\subsection{Robot control software}

Computer simulations and training are considered to be critical aspects in this project since the tasks they are used are in general critical in nature and should be carried out without any error. Creating a realistic computer simulation makes the operator to learn how the actual processes really look and feel. For the advanced operations like hazardous clean-up sites in nuclear environments, the real-time computer graphical simulations are compulsory. So, the development of teleoperator systems to be used in such environments will be considered as great and worthwhile achievements. For this purpose, software development is necessary and a graphical user interface (GUI) should be developed to make the user control the system with ease.

The main task of the program which is to be developed is to control Pontech SV203 controller. This SV203 controller can be programmed by Qbasic, C, Visual Basic or JAVA. Any of these programming languages can be used as long the SV203 controller receives the ASCII codes to perform the task. The computer interface of this SV203 
microcontroller is through RS232 serial port. Table 6.1 below describes the RS232 port [19].

Table 6.1 RS232 serial port male 9 pin connector

\begin{tabular}{|c|c|}
\hline $\begin{array}{c}\text { Male } \\
\text { RS232 }\end{array}$ & $\left(\begin{array}{c}(\odot)(9) \\
\text { DB9 }\end{array}\right.$ \\
\hline $\begin{array}{c}\text { Pin } \\
\text { Number }\end{array}$ & Direction of signal: \\
\hline 1 & Carrier Detect (CD) (from DCE) Incoming signal from a modem \\
\hline 2 & Received Data (RD) Incoming Data from a DCE \\
\hline 3 & Transmitted Data (TD) Outgoing Data to a DCE \\
\hline 4 & Data Terminal Ready (DTR) Outgoing handshaking signal \\
\hline 5 & Signal Ground Common reference voltage \\
\hline 6 & Data Set Ready (DSR) Incoming handshaking signal \\
\hline 7 & Request To Send (RTS) Outgoing flow control signal \\
\hline 8 & Clear To Send (CTS) Incoming flow control signal \\
\hline 9 & Ring Indicator (RI) (from DCE) Incoming signal from modem \\
\hline
\end{tabular}

We have decided to program in Visual Basic to communicate between the SV203 controller and the computer. The main reasons to choose Visual Basic is due to the fact that it is easy to program, is a powerful programming tool, as well as developing GUI interface and control through serial port are easy when compared to other programming platforms. The code is written in windows environment to be operated easily by the user. Many versions of the interfaces have been developed before the final version, which is presented in this work. 


\subsection{1-DOF FRMC Prototype simulation with mouse}

We have developed a program code in Visual Basic to show exactly how a 1-DOF FRMC operates. We have taken a PC mouse as our 1-DOF joystick (master robot), designed a car (representing a platform) on the PC monitor which is assumed to represent the slave robot and used an airplane icon (on the PC monitor screen) as an obstacle on the platform's motion path.

When the user moves the mouse (master robot) in the X-direction, the platform (slave robot) on the PC monitor moves accordingly. The program allows the user to set different positions for the obstacle. When the platform approaches the obstacle (plane icon) and if the user still tries to move the platform in the same direction, various color schemes (green, yellow, and red) warn the user of impending collision. This mimics force reflection on the actual prototype. The form views of the program depicting snapshots of the screen are shown in fig 6.1 and fig 6.2.

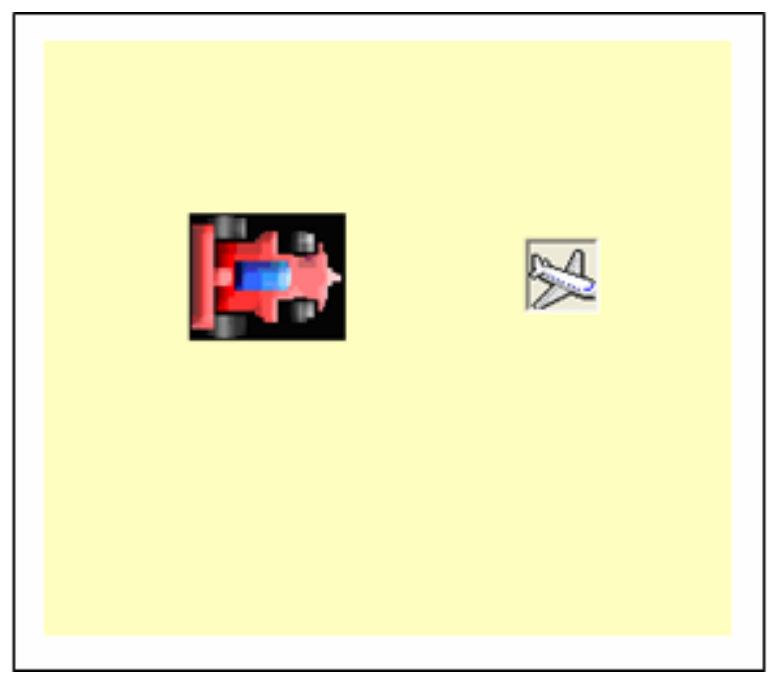

Figure 6.1 Form view when robot on the left is away from the obstacle represented by the plane icon 


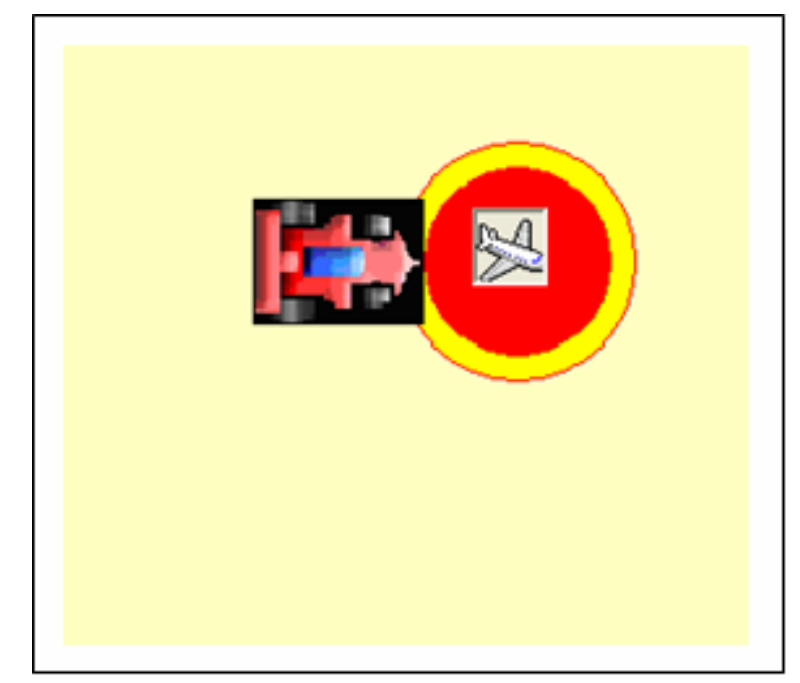

Figure 6.2 Form view when robot is close to the obstacle

\subsection{1-DOF FRMC Prototype simulation with SV203 controller}

We have replaced the PC mouse with a 1-DOF joystick which is operated by using a servo motor and the remaining parts are kept same. We have used a potentiometer to identify the shaft position of the servo motor, which is connected to the A/D port of the SV203. The GUI developed to control this SV203 is as shown in fig 6.3 below.

The GUI, which opens and closes the COM port, also controls servomotors and the A/D channels that are connected to the SV203 controller. When the 1-DOF joystick is made to move in either direction, the platform (slave robot) on the PC monitor moves accordingly. When the platform approaches the obstacle (the plane icon), various color schemes (green, yellow and red) warn the user of an impending collision. And if the user still tries to move the platform in the same direction, the operator feels the force reflection in the opposite direction so that the platform is moved away from the obstacle. 
After the platform is out of the collision area, force reflection is turned off and the current joystick position is set to the zero or home position.

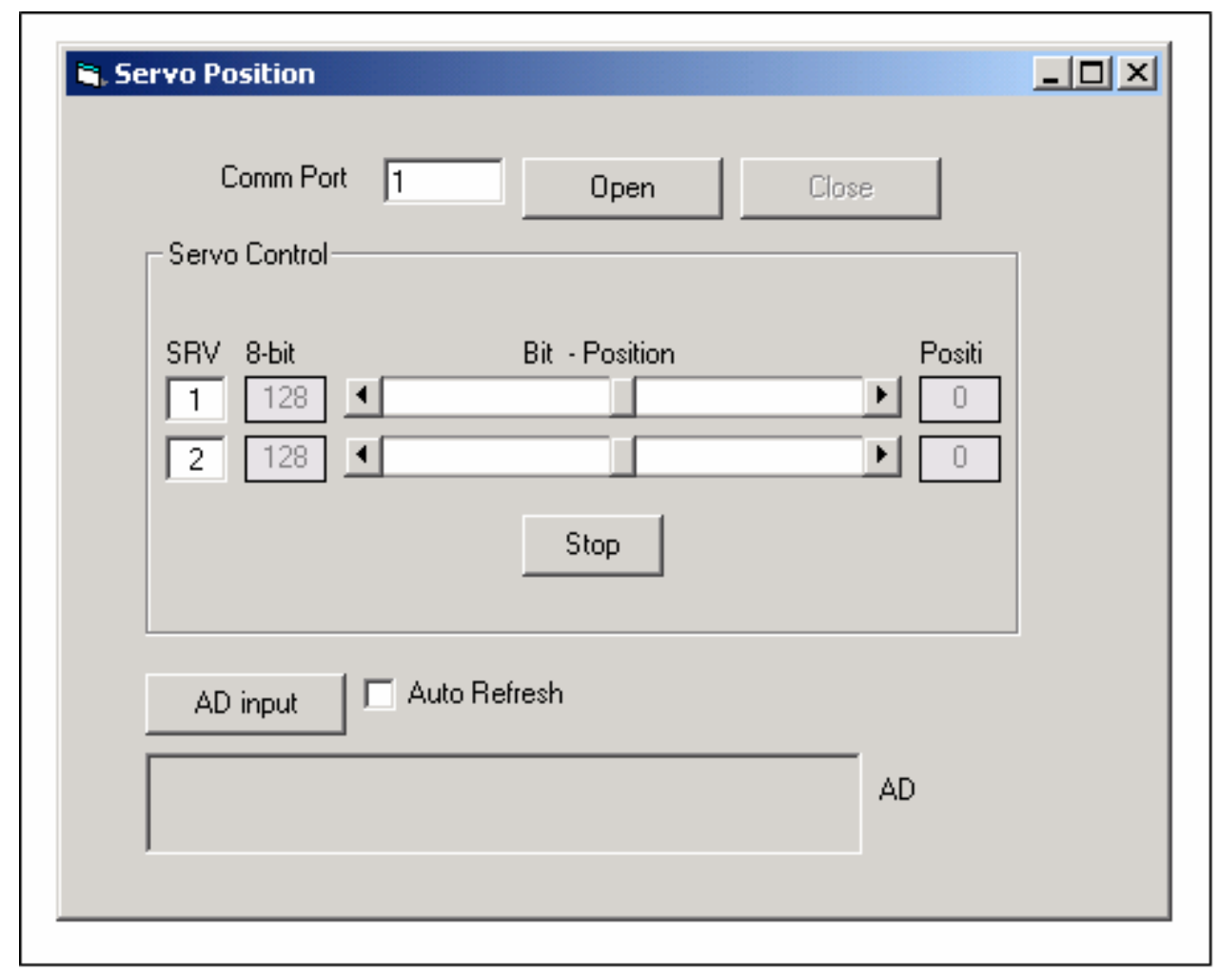

Figure 6.3 GUI developed to control SV203

Later we have updated the program so that the user can choose an icon to represent the slave robot either as a walking robot, car, or dinosaur. Similarly, obstacles can be represented on the screen in the form of a chair, football, doll, penguin, or aeroplane. The program also gives user the option to choose either position control (constant speed) or velocity control (variable speed) mode. The program also gives the user an option to specify a time delay, the amount of time delay, or no time delay. This program is also updated to let the user choose the direction of the slave robot motion (X 
or Y direction), which makes movements in the plane possible. The updated GUI is as shown in fig 6.4 below.

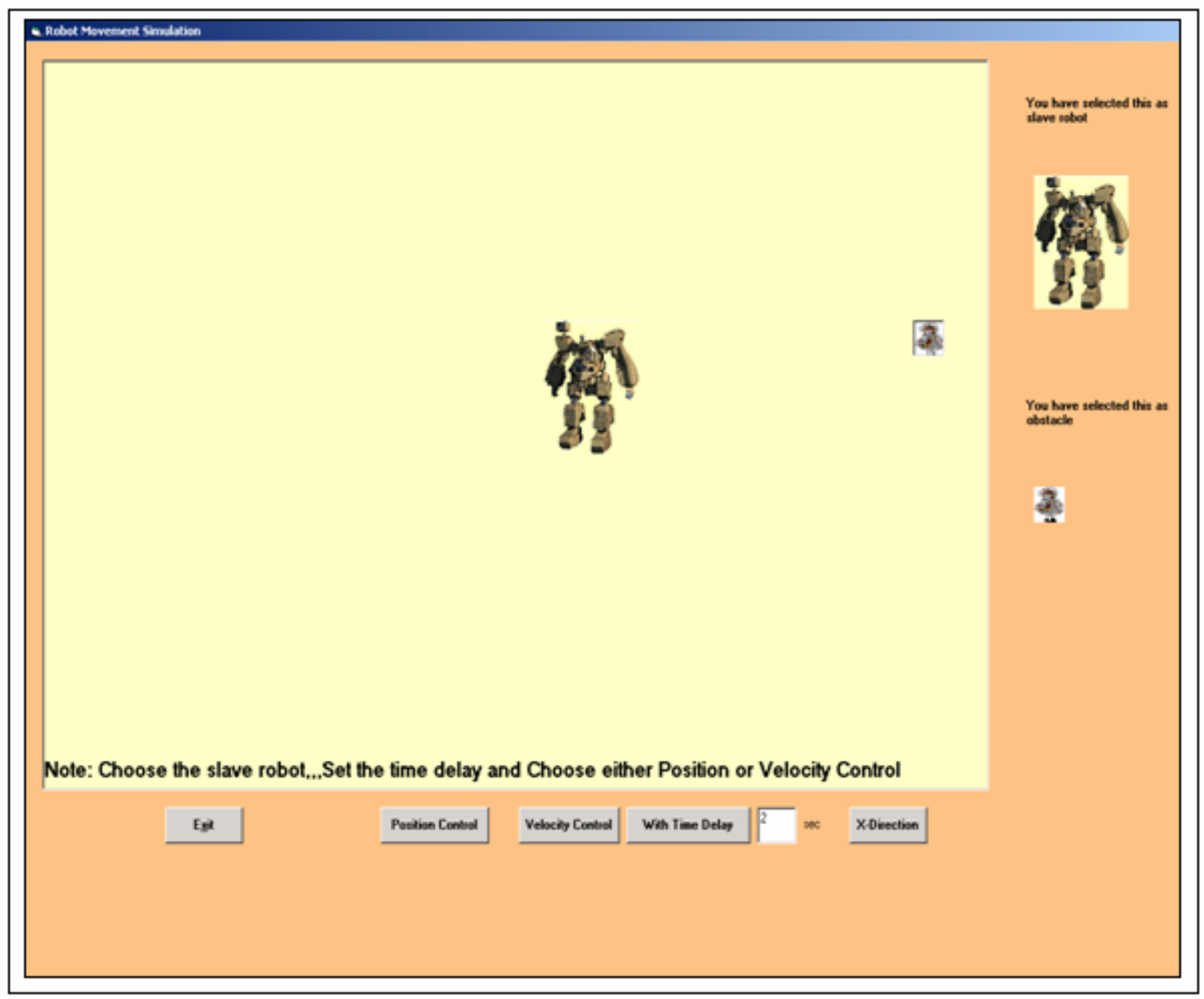

Figure 6.4 GUI of Visual Basic program

\subsection{Time delay in space applications}

The time delay for a signal to reach the moon from earth is on average $3 \mathrm{sec}$. Usually the loop delays (round-trip delays) are much greater; approaching $6 \mathrm{sec}$ in the case of earthorbiting space shuttle because of multiple up-down links (earth to satellite or the reverse) [30]. To incorporate time delay effects in remote control, we need the average end-to- 
end round-trip time between the slave and master robots. As the moon is very close to the earth compared to the other planets, the round-trip time delay in communications between the earth and moon is in the order of 3 to $6 \mathrm{sec}$. The space shuttles around the earth also come in the same zone of time delays.

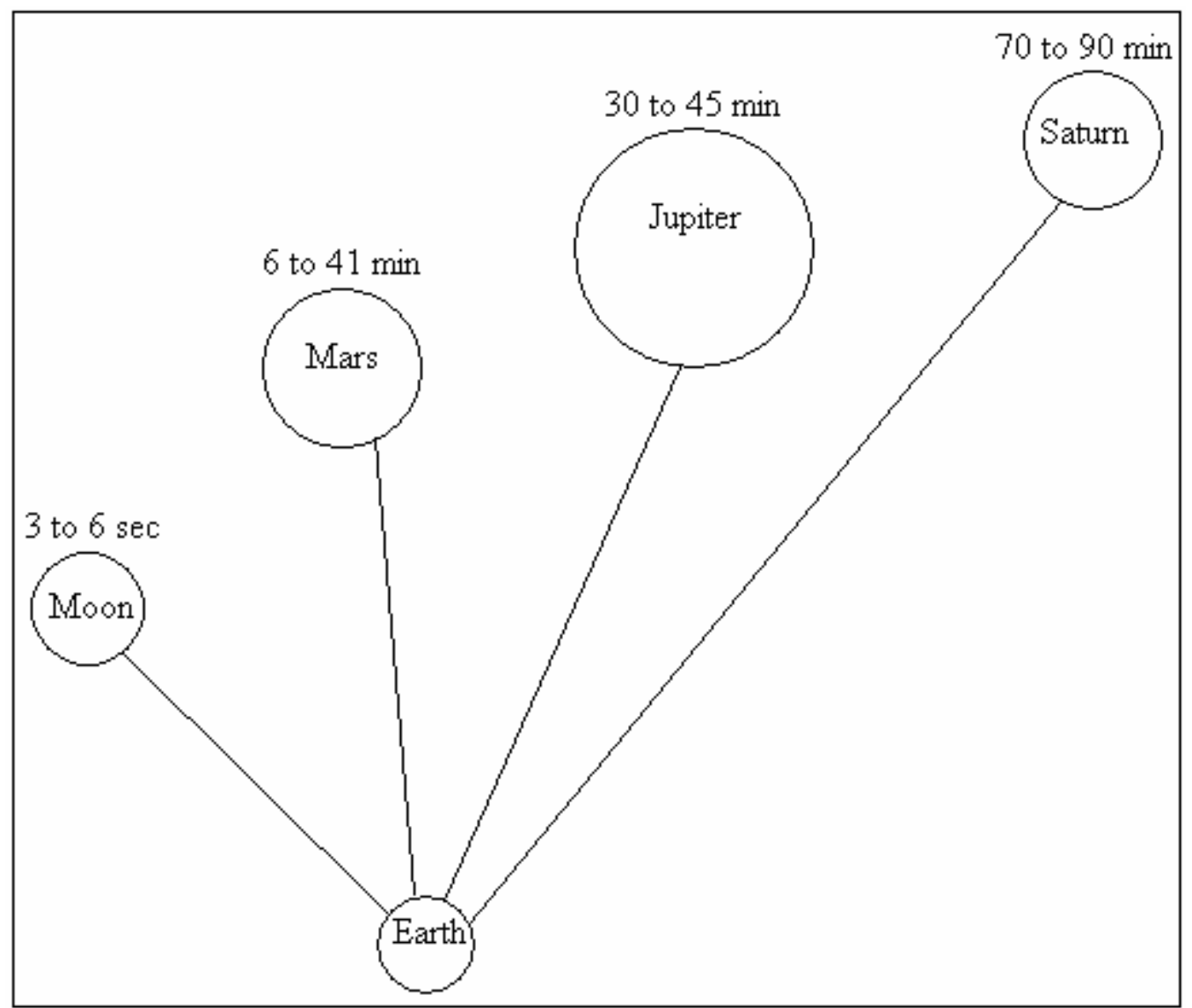

Figure 6.5 Round-trip communication time delays between the earth and planets

There are other planets around the earth, where there is a need for humans to communicate during space exploration missions. Depending upon the distance from the earth these time delays vary. Some of the timed delays in communications are mentioned below. Also, fig 6.5 shows the time delays from earth to different destinations in the 
universe. The end-to-end round-trip time delay for Mars-Earth communication network varies from 6 minutes to 41 minutes [32]. Also, the communication network delay for Jupiter-Earth varies approximately from 30 minutes to 45 minutes, and for Saturn-Earth communication the delay is approximately varies between 70 minutes to 90 minutes depending on their relative positions.

\subsection{Time delay experiments conducted with 1-DOF FRMC}

We have conducted several experiments with the 1-DOF FRMC on different users and with different time delays. The experimental setup is described below.

The remote site is represented on the computer screen as shown in fig 6.4.

- The user can position the obstacles among the indicated options

- Slave robot is chosen at the runtime by the user

- Time delay can be specified by the user

- Start and end positions are located at the diagonally opposing corners of the work area

The user is asked to operate the 1-DOF FRMC and move the slave robot from the predefined start position to the end position which are fixed (same) for all the users. With the same amount of time delay and same obstacle positions, each user has been tested and the amount of time taken for each user to reach the end position has been tabulated for different time delays varying from 0 to 8 sec. Each experiment was carried out on different days with different users to make sure that they do not get habituated to the path of the testing. With the gathered information, the effect of time delay on user's 
performance is studied. Table 6.2 shows the time required by each user to complete the task under different time delays.

Table 6.2 shows the experimental data

\begin{tabular}{|c|c|c|c|c|c|}
\hline $\begin{array}{c}\text { Time } \\
\text { delay } \\
\text { in sec }\end{array}$ & $\begin{array}{c}\text { Time taken } \\
\text { by User 1 } \\
\text { in sec }\end{array}$ & $\begin{array}{c}\text { Time taken } \\
\text { by User 2 } \\
\text { in sec }\end{array}$ & $\begin{array}{c}\text { Time taken } \\
\text { by User 3 } \\
\text { in sec }\end{array}$ & $\begin{array}{c}\text { Time taken } \\
\text { by User 4 } \\
\text { in sec }\end{array}$ & $\begin{array}{c}\text { Time taken } \\
\text { by User 5 } \\
\text { in sec }\end{array}$ \\
\hline 0 & 36.1 & 21.7 & 28.0 & 42.8 & 29.7 \\
\hline 1 & 58.3 & 43.5 & 41.7 & 57.2 & 63.3 \\
\hline 2 & 73.5 & 82.1 & 64.2 & 82.1 & 70.2 \\
\hline 3 & 138.4 & 150.3 & 119.4 & 157.2 & 138.1 \\
\hline 4 & 280.2 & 268.7 & 240.9 & 276.4 & 247.1 \\
\hline 6 & 427.5 & 435.2 & 389.2 & 440.4 & 413.5 \\
\hline 8 & 579.4 & 640.5 & 601.5 & 660.1 & 611.8 \\
\hline
\end{tabular}

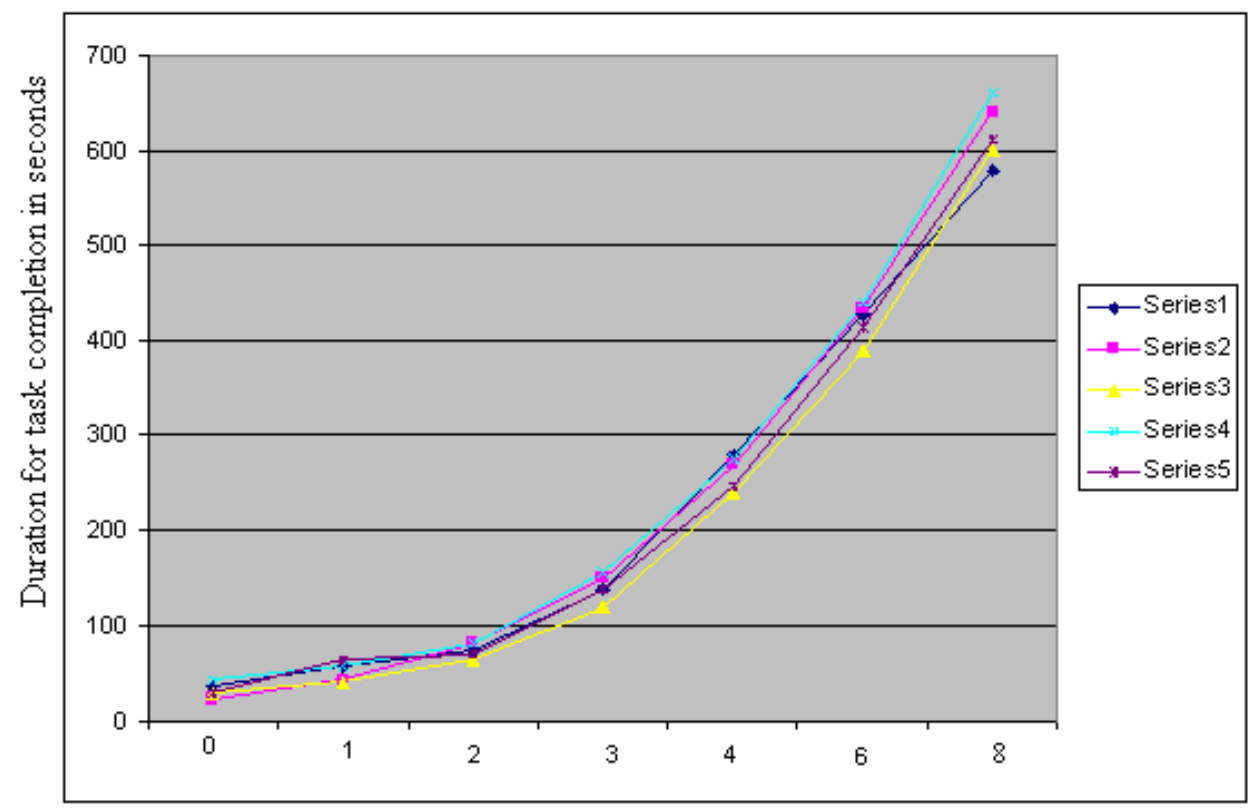

Time delay in seconds

Figure 6.6 Time delay trend for different users identified as series 1 through 5 
Fig 6.6 above plots the trend for each user's performance with respect to different time delays. As we can see from table 6.1 and fig 6.6 for the same start and end positions, each user has completed the required task in different timings, which depicts that the time delay will affect each user's performance differently. Hence, training users may help reduce this variation. Also, we have observed that the average time for task completion increases parabolically with the amount of time delay. For instance, the task has been completed on average in 74 seconds when 2 sec time delay was implemented in the experiments. The task is completed in 421 seconds when time delay becomes $6 \mathrm{sec}$ as can be seen in table 6.1 and fig. 6.6. Hence, in this example, a $4 \mathrm{sec}$ increase in time delay from 2 to $6 \mathrm{sec}$ introduces a 6 -fold increase in task completion time, which is quite high.

Finally, we would comment that the methods to enhance system performance caused by time delays are in general meant for delays in the order of $10 \mathrm{sec}$ at most and any larger time delays as indicated in fig. 6.5 are only for comparison purposes and no immediate remedy to such large time delays have been suggested. As the time delay increases, it would become harder for the operator to control the system and maintain the stability of the system. To reduce the negative effects of time delay, research has been carried out and one method known as the wave variable technique has been suggested to promise improvements. By using this technique, detrimental effects of time delay, for instance, in communicating between the earth and moon, is expected to be reduced.

\subsection{System integration}

Integrating the hardware and software for remote control systems is essential as it also requires integration and operation of its components over the network. Hence, in 
addition to the traditional hardware and software system design, we address broader system integration. In our system, integration means controlling the platform (PPRK platform modified for our project to represent the remote system) with the 1-DOF FRMC prototype through the software developed. It is important to develop the software in such a way to control the slave robot (modified PPRK) by the master robot (1-DOF FRMC) in real time as well as to incorporate a capability to control other remote systems.

In the present project, the structure of the sensing, planning, control system and the computer architecture has been designed for any task rather than for a specific task. The software interface developed is user-friendly, and this property of the system enables the user to complete the required task more efficiently. The two robots (master and slave) which are connected to two different computers (client and server) are operated by Winsock control (Visual Basic) through internet. In the system developed, the 1-DOF manual controller will get the feedback from the remote slave robot in two different methods; one by force reflection and the other by visual information received from the remote site through video camera attached to the slave robot.

In this visual feedback system, the video camera replaces the sensors of the force feedback system. The system with force feedback is capable of working in the remote site by seeing the simulations or the animated remote site on the screen of the computer to which it is attached while the system with the visual feedback is capable of seeing the real time video of the remote site through video camera attached to the slave robot. Integration of the modified PPRK platform, 1-DOF FRMC and the software makes the system more complex. However, the software developed is capable of controlling any 
robot built with the SV203 micro controller through internet by the 1-DOF FRMC developed.

\subsection{Winsock control}

The Winsock control provides easy access to Transfer Control Protocol (TCP) and User Datagram Protocol (UDP) network services. It can be used by Microsoft Access, Visual Basic, Visual $\mathrm{C}++$, or Visual FoxPro developers. By setting properties and invoking methods of the control, we can easily connect to a remote machine and exchange data in both directions.

\subsubsection{TCP}

The Transfer Control Protocol allows the user to create and maintain a connection to a remote computer. Using the connection, both computers can stream data between each other.

In client server applications, while creating a client application, we must know the server computer's name or IP address (RemoteHost property), as well as the port (RemotePort property) on which it will be "listening" to. Then invoke the Connect method.

In order to create a server application, we should set a port (LocalPort property) on which to listen, and invoke the Listen method. When the client computer requests a connection, the ConnectionRequest event will occur. To complete the connection, the system invokes the Accept method within the ConnectionRequest event. Once a connection has been made, either computer can send and receive data. To send data, we need to invoke the 
SendData method. Whenever data is received, the DataArrival event occurs. To retrieve data, we have to invoke the GetData method within the DataArrival event.

\subsubsection{UDP}

The User Datagram Protocol (UDP) is a connectionless protocol. Unlike TCP operations, computers do not establish a connection. Also, a UDP application can be either a client or a server. To transmit data in client server applications, first set the client computer's LocalPort property. The server computer then needs only to set the RemoteHost to the Internet address of the client computer, and the RemotePort property to the same port as the client computer's LocalPort property, and invoke the SendData method to begin sending messages. The client computer then uses the GetData method within the DataArrival event to retrieve the sent messages.

\subsubsection{Winsock properties}

Winsock enables the user to create clients and servers using the same control. This dual functionality enables the user to specify through property setting the type of application we will be building. Some of the important properties of the control are as follows:

- BytesReceived property

This property returns the number of bytes currently in the receive buffer. The value returned is a long integer. 
- LocalHostName property

The LocalHostName property returns the name of the local host system. The value returned is a string.

- LocalIP property

The LocalIP property returns the local host system IP address in the form of a string, such as 11.0.0.127.

- LocalPort property

This property returns or sets the local port number. This can be both "read from" and "written to" and is available at both design time and runtime. The value returned is a long integer.

- RemoteHost property

The RemoteHost property returns or sets the remote host. This can be both "read from" and "written to" and is available both in design time and runtime. The value returned is a string and can be specified as an IP address.

- $\quad$ State property

This returns the state of the control as expressed by an enumerated list. This is a readonly property.

\subsubsection{Winsock methods}

- Accept method

It accepts the request for connection from the client system. For this method to be used, the control must be in the listening state. 
- Close method

The Close method terminates a TCP connection from either the client or server applications.

- GetData method

GetData is the method that retrieves the current block of data from the buffer and then stores it in a variable of the variant type.

- PeekData method

The PeekData method operates in a fashion similar to the GetData method. However, it does not remove data from the input queue.

- Listen method

This is invoked on the server application to have the server application wait for a TCP request for connection from a client system.

- SendData method

This method dispatches data to the remote computer. It is used for both the client and server systems.

\subsection{Operating the system software}

The process of operating the system software is discussed below. There are two computers used in this process and are named as client and server. The 1-DOF manual controller (master robot) is connected to the server side and the modified PPRK or the 1DOF slider bar (slave robot) is connected to the client side.

First the client side computer should be connected to the server side computer by entering the IP address of the server side computer. Once they are connected, both the 
computers are able to send and receive the data. The 1-DOF manual controller (master robot) has to be loaded on the server side. Similarly the modified PPRK or 1-DOF FRMC (slave robot) has to be loaded on the client side. As both the systems, master robot and the slave robot use SV203 micro controller, they have to be connected to the computers through RS232 port. Fig 6.7 shows the basic process of the system developed.

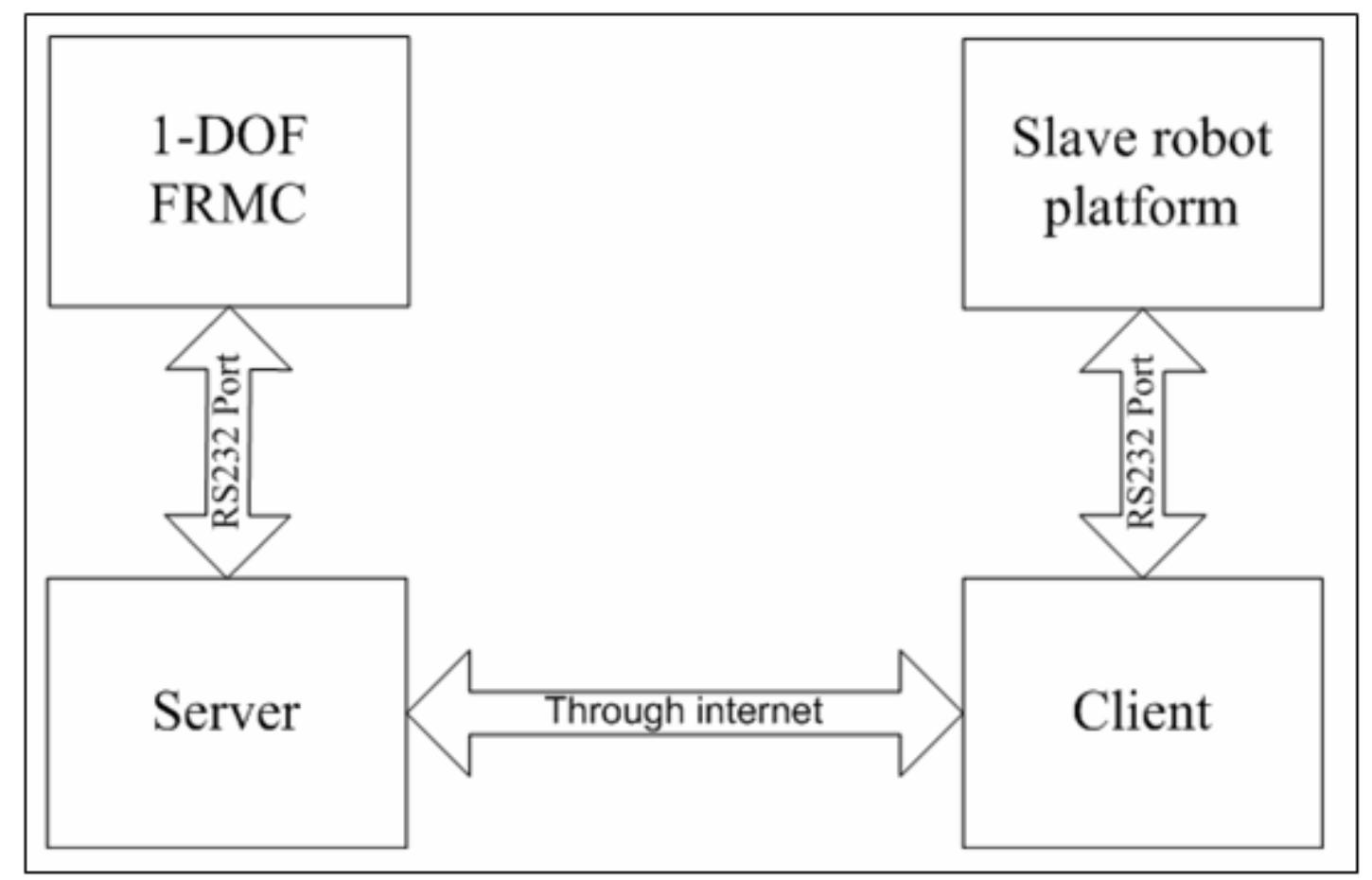

Figure 6.7 Process of the system developed

The position of the joystick is tracked by the potentiometer attached to it and that AD reading is sent to the server side computer through SV203 controller and RS232 port. The AD reading from the server side computer is sent to the client side computer through internet. The client side computer sends the AD reading to the SV203 controller through RS232 port, which compares the AD reading with the condition and will move the servo attached to the slave robot accordingly. At the same time the reading of the IR sensor on 
the remote site is sent to the client side computer through SV203 controller and will in turn send that $\mathrm{AD}$ reading to the server side computer through internet. The $\mathrm{AD}$ reading of the IR sensor is checked with some conditions and will make the SV203 controller to decide whether to call force reflection on the joystick or not.

As this process is continuing the $\mathrm{AD}$ reading of the potentiometer is sent to the server side computer and on the screen of the server side computer the user can see the simulation of the remote site environment and feels as if he is working in the real environment. While operating joystick the platform on the computer screen moves accordingly, and as it approaches any obstacle on its path and if the user still tries to move the joystick in the same direction, various color schemes (red, yellow and green) warn the user of impending collision which also sends the force reflection command on the actual 1-DOF FRMC.

\subsubsection{Video streaming}

Here in this process, the user operating slave robot will see live video of the environment where the slave robot is operated through the video camera attached to the slave robot. The system settings of this operation are described as follows. First the client side computer should be connected to the server side computer by entering the IP address of the server side computer. Once they are connected, both the computers are able to send and receive the data. The 1-DOF manual controller (master robot) has to be loaded on the server side. Similarly the modified PPRK or 1-DOF FRMC (slave robot) has to be loaded on the client side. As both the systems, master robot and the slave robot use SV203 micro controller, they have to be connected to the computers through RS232 port. A web 
camera should also be connected to the computer USB port and is kept on the slave robot as shown in the fig 6.8 below.

The position of the joystick is tracked by the potentiometer attached to it and that AD reading is sent to the server side computer through SV203 controller and RS232 port. The AD reading from the server side computer is sent to the client side computer through internet. The client side computer sends the AD reading to the SV203 controller through RS232 port and will move the servo attached to the slave robot accordingly.

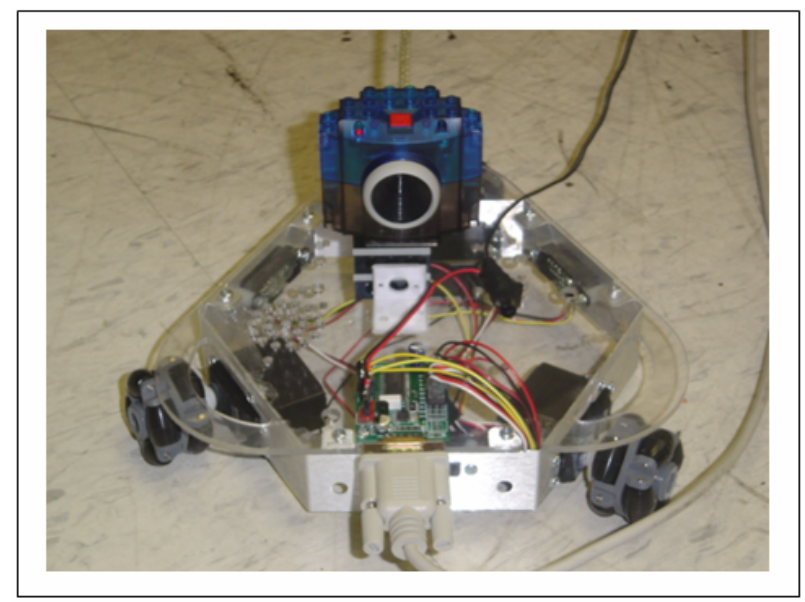

Figure 6.8 Camera attached to the PPRK

As this process is in progress the user can see the live video of the remote site through the web camera attached to the slave robot at remote site. Fig 6.9 and 6.10 shows the images of the video frames at client and server that are transferred while operating the software. 


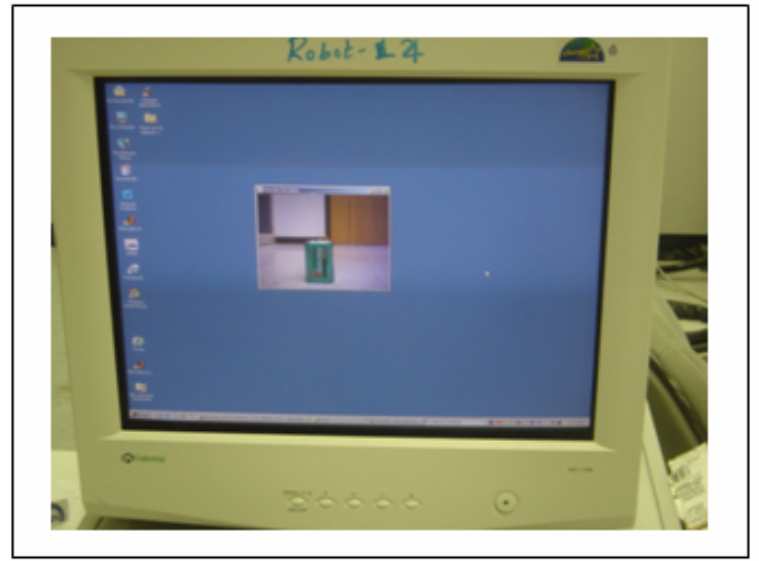

Figure 6.9 Webcam at client

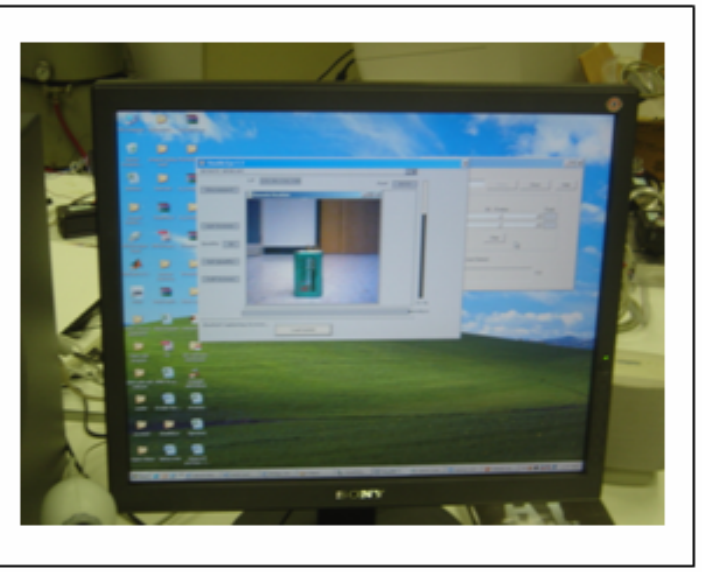

Figure 6.10 Webcam at server 


\section{CHAPTER 7}

\subsection{RESULTS AND DISCUSSIONS}

A teleoperator system extends the intelligence and capabilities of humans and robots by force and visual feedback information. A 1-DOF manual controller has been designed and developed for this purpose which has both force and visual feedback from the remote environment (teleoperation). Design process includes motor and controller selection, and the types of transmission system used are important criteria to be considered in mechanical design of the system. Design process should also consider minimization of weight, size and, if appropriate, its cost since most of the previously designed forcereflecting manual controllers are bulky, complex and expensive, or are cheap and inaccurate designs meant for computer games.

\subsection{Conclusions}

In an effort to develop the 1-DOF FRMC system which meets all the above described conditions, chapter 2 reviews some of the previous designs of the force-reflecting manual controllers which include the mechanical design techniques and the control strategies. That chapter also reviews the different conceptual designs proposed for the development of the 1-DOF FRMC with advantages and disadvantages of each design.

In chapter 3, the major components, such as the actuators and sensors used in the design of 1-DOF FRMC system have been reviewed. All of the available actuators and different types of sensors available in the market are reviewed, and the suitable actuator and sensors are selected by comparing them with the rest of the available ones. 
In chapter 4, the most important component in the design of 1-DOF FRMC, micro controller, is discussed. The different available micro controllers are reviewed and the final selection is presented.

In chapter 5, 1-DOF FRMC prototype is designed and developed to demonstrate the principle of 1-DOF FRMC. All of the important components used in the design of the 1-DOF FRMC have been described with their specifications. The final assembly of the system has been developed.

1-DOF platform has been constructed to check whether the prototype is working properly or not. For this purpose, a 1-DOF slider bar has been designed and constructed for use as a remote platform. The components used to develop the 1-DOF slider bar have been described in this chapter.

As a secondary remote platform, PPRK mobile robot model has been developed and its parts specifications have been listed. PPRK robot has been modified to enable its integration into the 1-DOF FRMC. This platform essentially consists of three continuous rotational servo motors, three IR (infrared) sensors and an SV203 controller.

In chapter 6 , the software developed to control the 1-DOF FRMC has been briefly described. The software tool used for this purpose is Visual Basic. First, the simulation with the mouse as 1-DOF manual controller has been developed. Later, the mouse has been replaced by the 1-DOF FRMC and the simulation is carried out in the same way by utilizing the Visual Basic software.

Integration of 1-DOF FRMC with the developed platforms, i.e., 1-DOF slider bar and the modified PPRK mobile robot, have been described. In this simulation software, we have also developed visual feedback in addition to force reflection. This software was 
also developed in Visual Basic. Hence, the developed system has both force and visual feedback. The software developed in Visual Basic is generic, which allows the 1-DOF FRMC control any robot built with SV203 micro controller through the internet (teleoperation).

Laboratory experiments have been conducted on the developed 1-DOF FRMC system to test the effect of time delays on the human performance. These experiments have been conducted on different users with varying time delays and the effects of these time delays have been described.

\subsection{Recommendations}

Although the goal of this work has been achieved, looking back at the work done, we can always identify areas where further improvements can be introduced. In this work instead of using an umbilical cord for the power supply, we could have placed a battery pack on the platform. The 1-DOF FRMC interface with the system uses RS232 port through a serial port wire, that can also be replaced with a wireless transmitter and receiver, which makes the system more efficient. The modified system can then be easily transportable and will be the same size as the one developed but it would not have any wires attached to its housing assembly.

\subsection{Future work}

The robot movement is not completely synchronized with the feedback received from the remote webcam in case of visual feedback system. There is a time delay of 2 to 4 seconds observed between the real time video and the video received at manual controller site. 
This is due to the network problem; this limitation can be solved by using a high-speed network.

Higher degrees of freedom, such as 2-DOF and 3-DOF, manual controllers can be developed. A 2-DOF can be easily developed from the design we currently have presented. By attaching another RC servo at the other end of the mechanism, which is used to measure the shaft position of the servo, we can develop a 2-DOF FRMC.

Moreover, the slave robot movement is restricted to the maximum length of RS232 serial cable and the USB cable (web camera). This restriction can be avoided by using a wireless transmitter and receiver at the remote site, which makes the slave robot to overcome the limited boundary conditions.

Also, virtual reality (VR) environment may be developed. Various components such as the VR unit, microphone and speakers can also be integrated with the software developed, which makes the system more useful and appealing.

Furthermore, advanced control techniques may be developed to overcome the time delay problem. The wave variable technique is one such promising method currently being evaluated at FIU's Robotics and Automation Laboratory. 


\section{References}

1. G. Messadie, "Great Inventions Throughout History,” Chambers, Edinburgh, 1988.

2. Philip John McKerrow, "Introduction to Robotics," University of Wollongong, Australia, 2001. ISBN: 0-201-18240-8.

3. Pattaraphol Batsomboon, "Design and Construction of a Portable Force-reflecting Manual Controller for Teleoperation Systems," Master Thesis, Department of Mechanical Engineering, Florida International University, 1998.

4. J.V. Draper, "Teleoperator for Advanced Manufacturing: Applications and Human Factors Challenges," The International Journal of Human Factors in Manufacturing, Vol. 5, No. 1, pp. 53-85, 1995.

5. T. Massie, and J. Salisbury, "The PHANToM Haptic Interface: A Device For Probing Virtual Objects," Proceedings of the ASME Winter Annual Meeting Symposium on Haptic Interfaces for Virtual Environment and Teleoperator Systems, DSC-Vol. 55-1, pp. 295-300, New York, 1994.

6. R. L. Williams, M. Srivastava, R. Conaster, and J. N. Howell, "Implementation and Evaluation of a Haptic Playback System," Haptics-e: The Electronic Journal of Haptics Research, co-sponsored by the IEEE Robotics and Automation Society, Vol. 3, No. 3, May 3, 2004.

7. M Bouzit, G. Popescu G. Burdea, and R. Boian, 2002, "The Rutgers Master II-ND Force Feedback Glove," IEEE Virtual Reality (VR) 2002 Haptics Symposium, Orlando FL, March 2002.

8. B. Eberman and B. An. EXOS Research on Force Reflecting Controllers. SPIE Telemanipulator Technology, 1833:9--19, 1992.

9. A. Kamerkar, and T. Kesavadas, "Touch Based Interactive NURBS Modeler using a Force/Position Input Glove," ASME Design and Technology Conferences, Salt Lake City, Utah, Sept. 2004.

10. P. Buttolo, "Characterization of Human Pen Grasp with Haptic Displays," Ph.D. Dissertation, University of Washington, Department of Electrical Engineering, June 1996.

11. G. Niemeyer, Kuchenbecker, K. J., Bonneau, R., Mitra, P., Reid, A. M., Fiene, J., and Weldon, G. "THUMP: an Immersive Haptic Console for Surgical Simulation and Training", presented at MMVR: Medicine Meets Virtual Reality, Newport Beach, California, January $15-17,2004$. 
12. Burdea, J. Zhuang, E. Roskos, D. Silver and N. Langrana, "A Portable Dextrous Master with Force Feedback," Presence, Vol. 1. No.1, pp. 1827, March 1992.

13. Futaba Servo Motors, available http://www.futabarc.com/servos/futm0029.html, last accessed September 2004.

14. D.McCloy, D.M.J.Harris, " Robotics; An Introduction”, halsted Press, New York, NW, 1986

15. P. Batsomboon, S. Tosunoglu, D. W. Reppeger, "Design and Construction of a Portable Force-Reflecting Manual Controller for Teleoperation Systems," 1997 Florida Conference on Recent Advances in Robotics, Florida International University, Miami, Florida, pp. 1-7, April 10-11, 1997.

16. Chandrasekar R. Puligari, M. I. Can Dede, S. Tosunoglu, and D. W. Repperger, "Development of a Force-reflecting Manual Controller Prototype for Teleoperation," ASME Southeastern Region XI Technical Journal, Volume 3, Number 1, April 2004; also presented at the ASME Southeastern Region XI Technical Conference, Mobile, Alabama, April 2-4, 2004.

17. J. Blanch, and S. Tosunoglu, "Control of Mobile Platforms via Hand-Held PDAs," The $15^{\text {th }}$ Florida Conference on Recent Advances in Robotics and Robot Showcase, Florida International University, Miami, Florida, May 23-24, 2002.

18. N. Chopra, M.W. Spong, S. Hirche, and M. Buss, "Bilateral Teleoperation over the Internet: the Time Varying Delay Problem." Proceedings of the American Control Conference, Denver, CO, June 4-6, 2003.

19. Taltech Instrumental Software, http://www.taltech.com/resources/intro-sc.html\#232c, accessed October 2004.

20. K. Mukhar, D. Johnson, "The Ultimate Palm Robot,” McGraw-Hill, California, 2003, ISBN: 0-07-222880-6.

21. Lynxmotion Copmany, http://www.lynxmotion.com/Product.aspx?productID=214\&CategoryID=52, accessed October 2004.

22. Pololu Robot-kits website, http://www.pololu.com/products/pololu/0290/, accessed October 2004.

23. Lynxmotion Copmany, http://www.lynxmotion.com/Product.aspx?productID=49\&CategoryID=7, accessed October 2004. 
24. Active Robots Website, http://www.active-robots.com/products/controllr/index.shtml, accessed October 2004.

25. http://www.mcmanis.com/chuck/robotics/controllers/miniboard.htm, accessed October 2004.

26. Acroname Inc, http://www.acroname.com/robotics/parts/S1-GP-BRD.html, accessed October 2004.

27. J. Blanch, and S. Tosunoglu, "Servo and Sensor Control of Small Mobile Platforms," ASME Southeastern Region XI Technical Journal, Volume 2, Number 1, April 2003; also presented at the ASME Southeastern Region XI Technical Conference, Miami, Florida, April 4-5, 2003.

28. J. Blanch, and S. Tosunoglu, "Hand-Held Computers as Mobile Platform Controllers," Florida Conference on Recent Advances in Robotics, Florida State University, Tallahassee, Florida, May 10-11, 2001.

29. H. J. Bosshard, I. Birrer, V. Cechticky, A. Rohlik, A. H. Glattfelder and W. Schaufelberger, "Teaching of Software for Control Systems Using Handheld and Laptop Computers and Simple Robots,"Proceedings 33rd International Symposium Ingenieurpädagogik, Fribourg, Switzerland, September 27-October 1, 2004.

30. T.B. Sheridan, "Space Teleoperation through Time Delay: Review and Prognosis," Proceedings of IEEE Transactions on Robotics and Automation, Volume 9, Number 5, October 1993.

31. http://users.ece.gatech.edu/ ifang/deepspace/transport.html, accessed on November 2004.

32. NASA website, http://cmex-www.arc.nasa.gov/SiteCat/sitecat2/pathfind.htm, accessed on November 2004. 


\section{APPENDICES}

APPENDIX A - VISUAL BASIC 1-DOF FRMC CONTROL PROGRAM (SIMULATION)

APPENDIX B - VISUAL BASIC 1-DOF FRMC CONTROL PROGRAM FOR TELEOPERATION OVER INTERNET

APPENDIX C - VISUAL BASIC 1-DOF MANUAL CONTROLLER CONTROL PROGRAM FOR TELEOPERATION OVER INTERNET (VISUAL FEEDBACK) 


\section{APPENDIX A - VISUAL BASIC 1-DOF FRMC CONTROL PROGRAM (SIMULATION)}

We have four modules and four forms for this program. We will list all the modules first and then forms.

- MODULE PontechComm (PontechComm.bas)

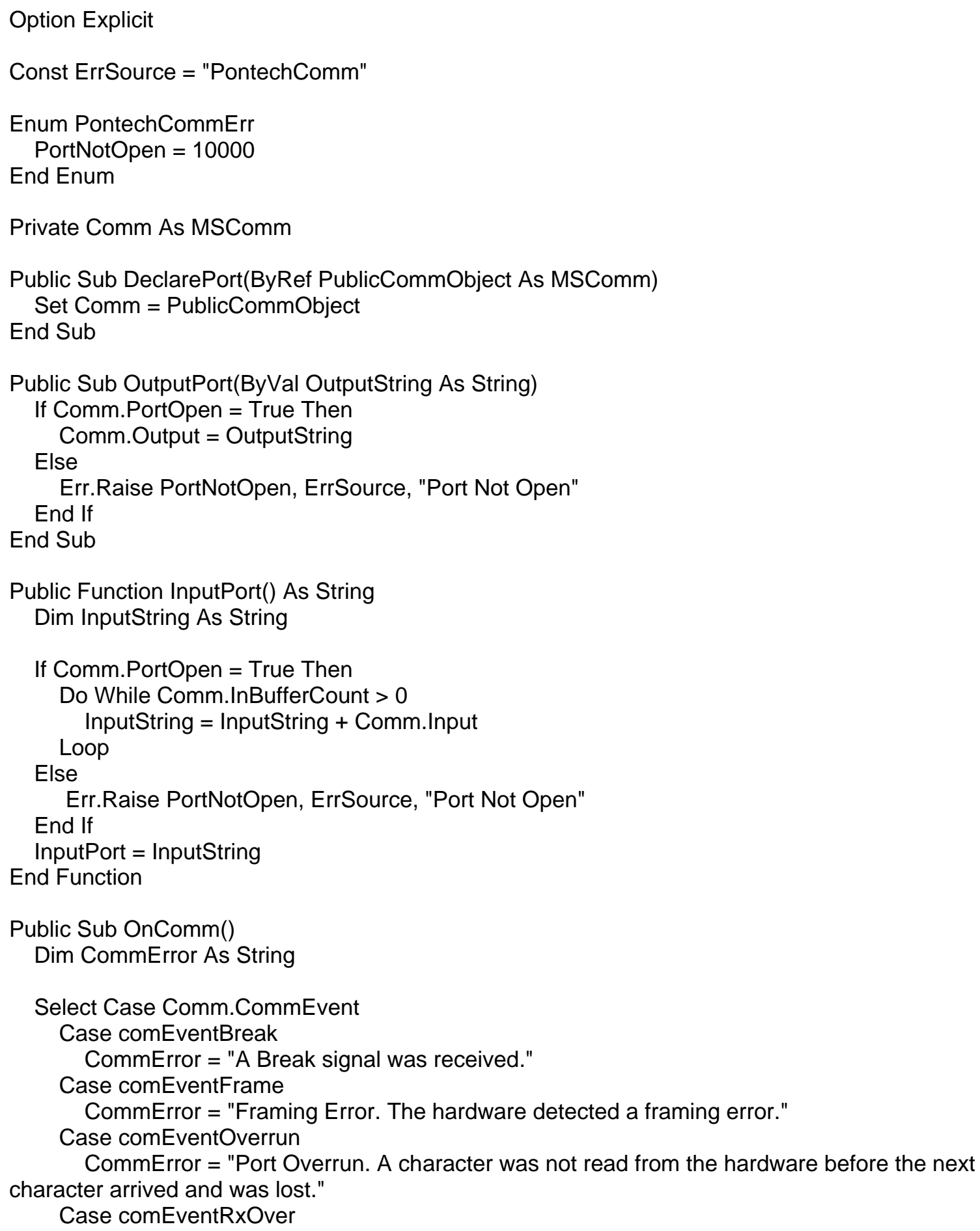


CommError = "Receive Buffer Overflow. There is no room in the receive buffer." Case comEventRxParity CommError = "Parity Error. The hardware detected a parity error."

Case comEventTxFull

CommError = "Transmit Buffer Full. The transmit buffer was full while trying to queue a character."

Case comEventDCB

CommError = "Unexpected error retrieving Device Control Block (DCB) for the port."

Case comEvSend buffer."

CommError $=$ "There are fewer than Sthreshold number of characters in the transmit

Case comEvReceive

CommError = "Received Rthreshold number of characters. This event is generated

continuously until you use the Input property to remove the data from the receive buffer."

Case comEvCTS

CommError = "Change in Clear To Send line."

Case comEvDSR

CommError = "Change in Data Set Ready line. This event is only fired when DSR changes from 1 to $0 . "$

Case comEvCD

CommError = "Change in Carrier Detect line."

Case comEvRing

CommError = "Ring detected. Some UARTs (universal asynchronous receivertransmitters) may not support this event."

Case comEvEOF

CommError = "End Of File (ASCII character 26) character received."

Case Else

CommError = "Unknown Communications Error"

End Select

MsgBox CommError

End Sub

- MODULE SV203 (SV203.bas)

Option Explicit

Const ErrSource = "SV203"

Enum SV203Err

InvalidServoNumber $=10000$

InvalidServoPosition

InvalidADPin

End Enum

Public Function ReadADC(ByVal AD_Pin As Integer) As Integer

Dim Command As String

Dim Result As String

If AD_Pin < 1 Or AD_Pin > 5 Then

Err.Raise InvalidADPin, ErrSource, "Invalid AD Pin Number"

End If

Command $=$ Command + "AD" + CStr(AD_Pin $)+\operatorname{Chr}(13)$

Call PontechComm.OutputPort(Command)

Call Utility_Time.Sleep(0.1)

Result $=$ PontechComm .InputPort 


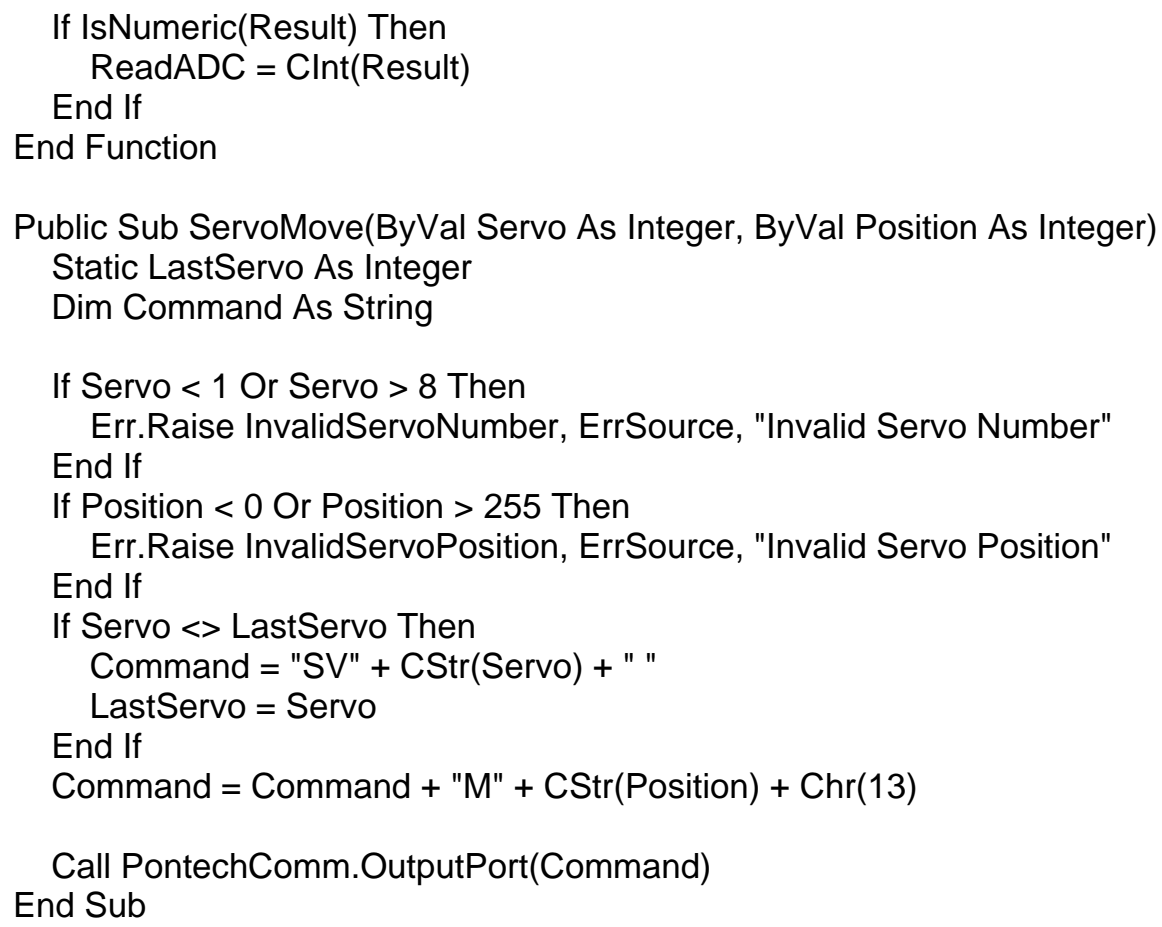

- MODULE STP100 (STP100.bas)

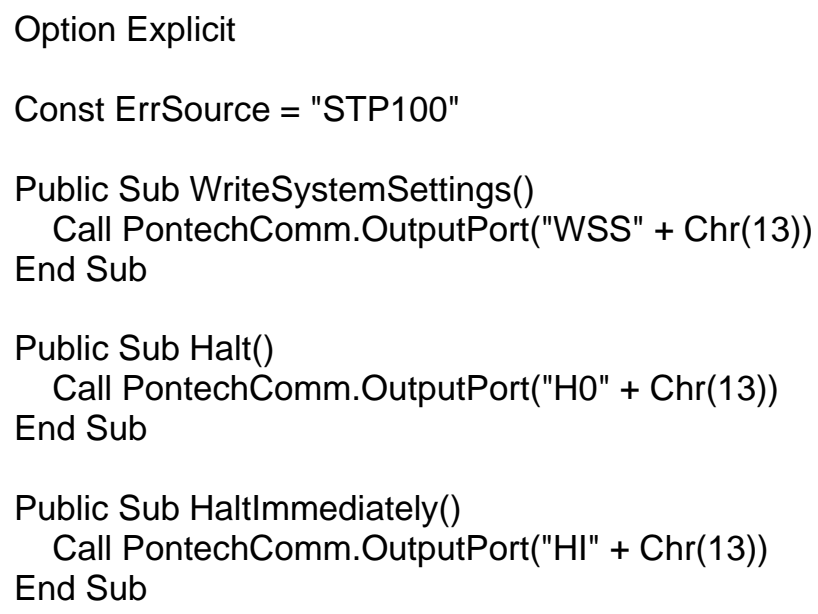




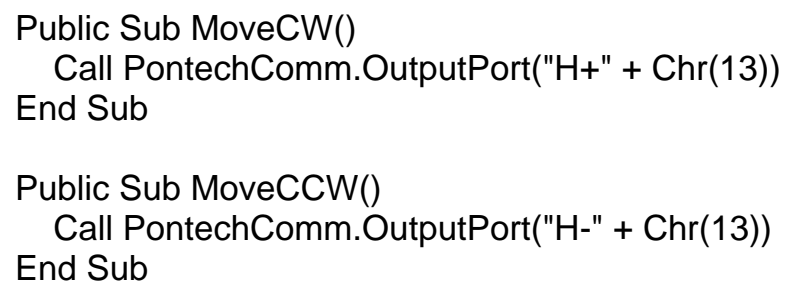




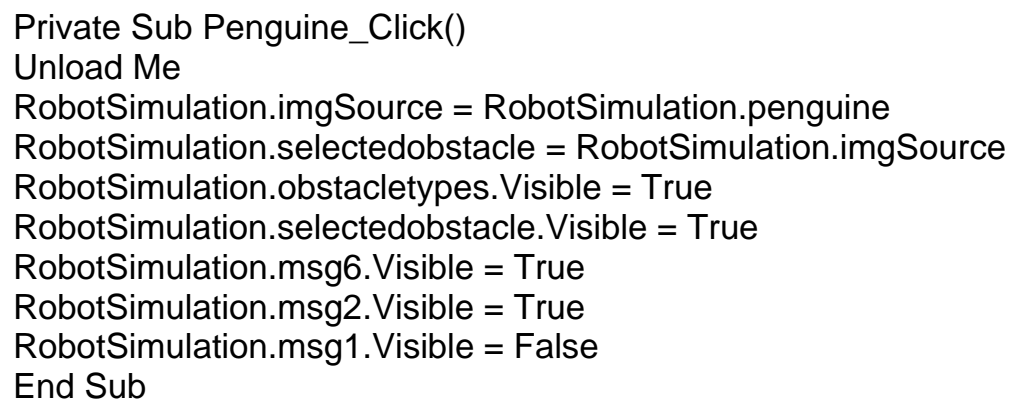

'Decleration Section

Dim amountoftimedelay, delaystart As Single

' FUNCTION THAT QUITS THE PROGRAM

Private Sub cmdExit_Click()

End

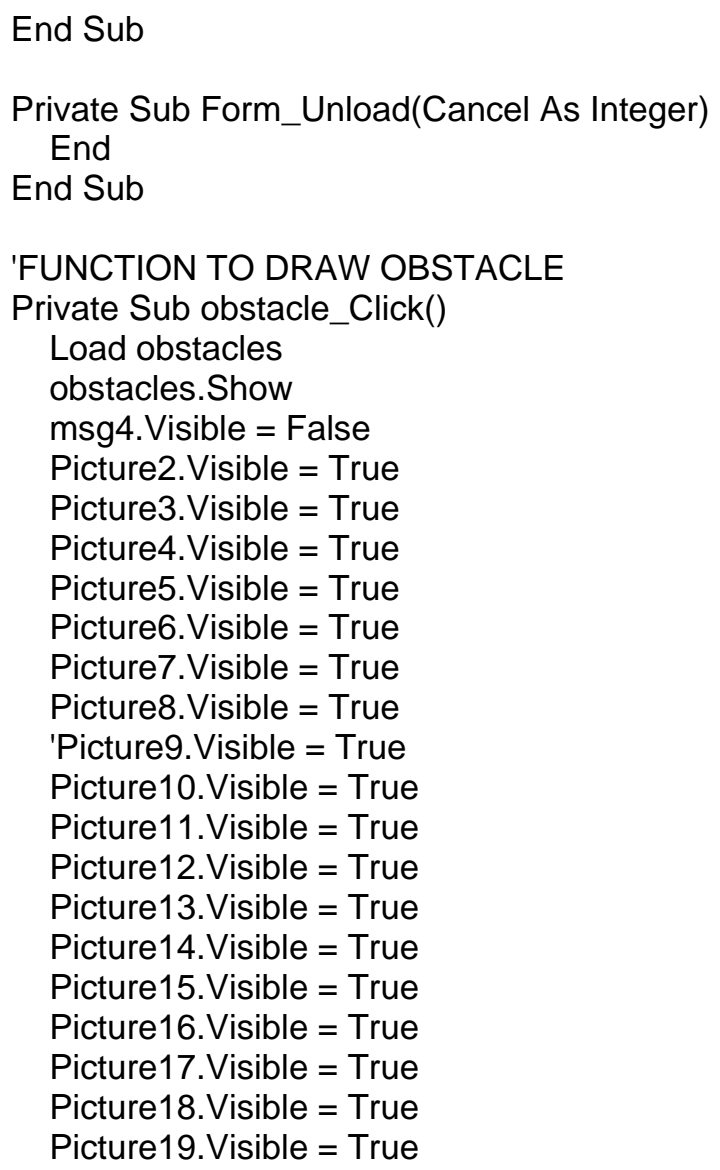




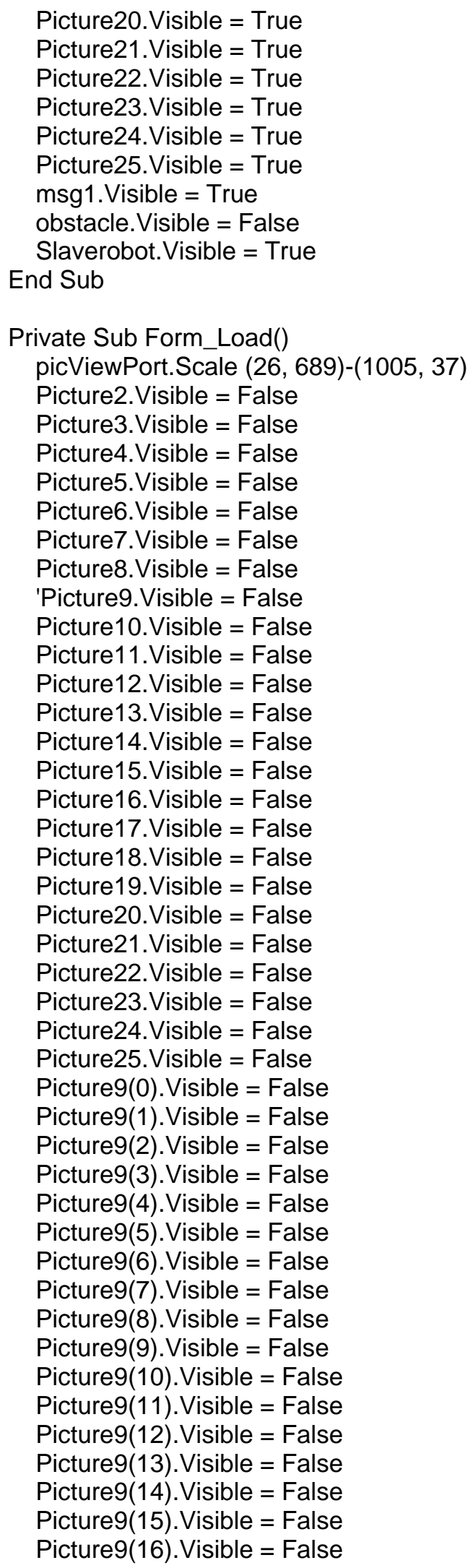




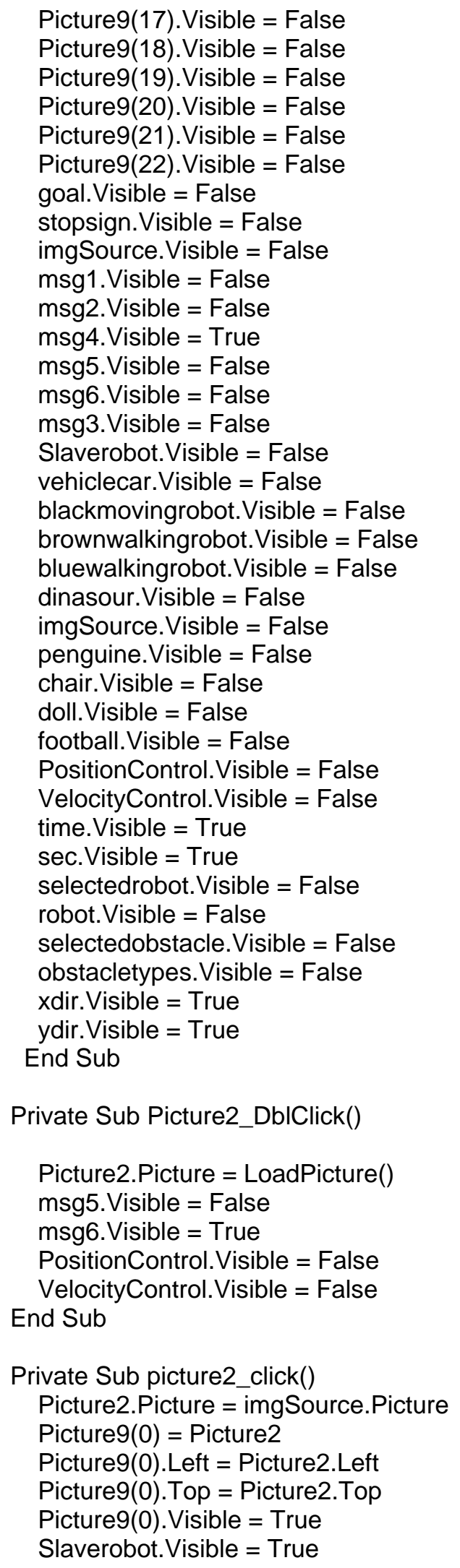




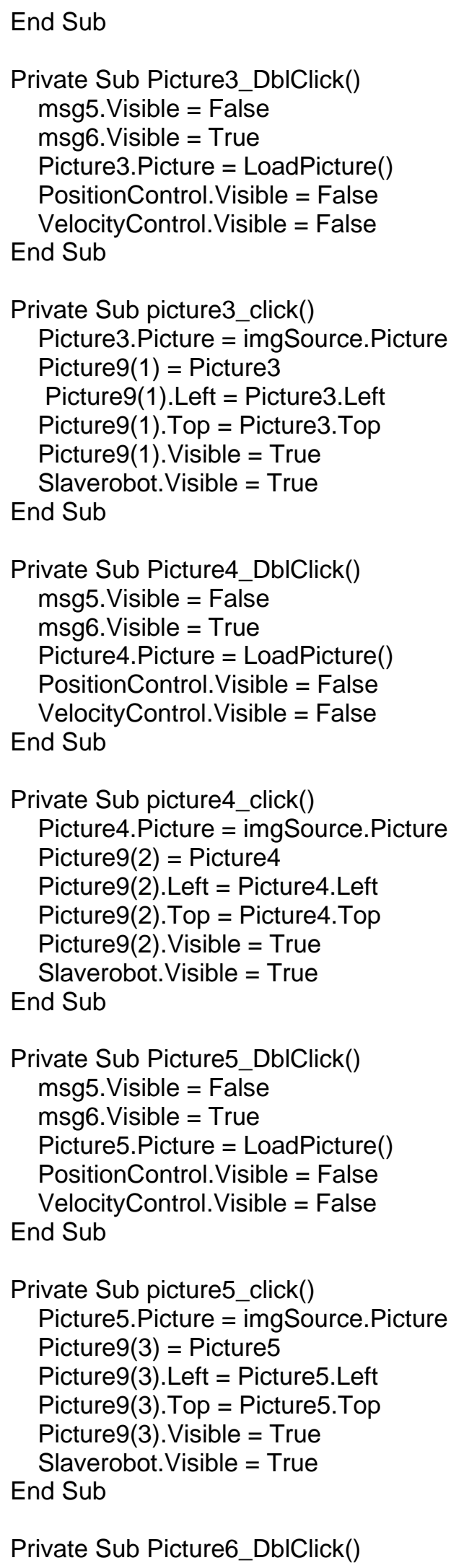

Private Sub Picture6_DblClick() 


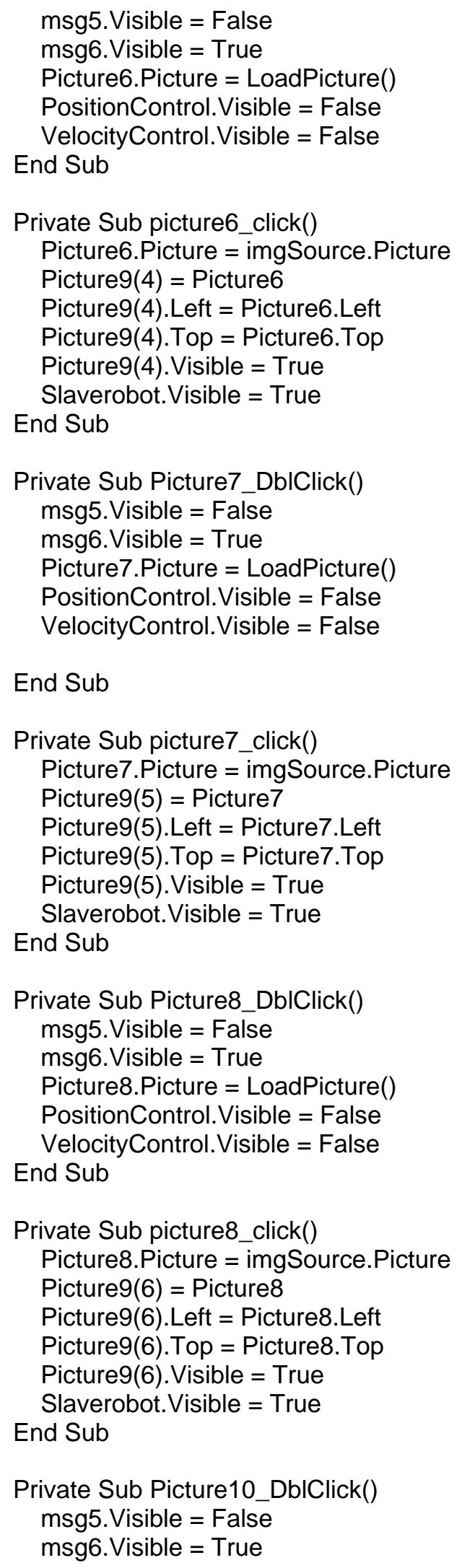




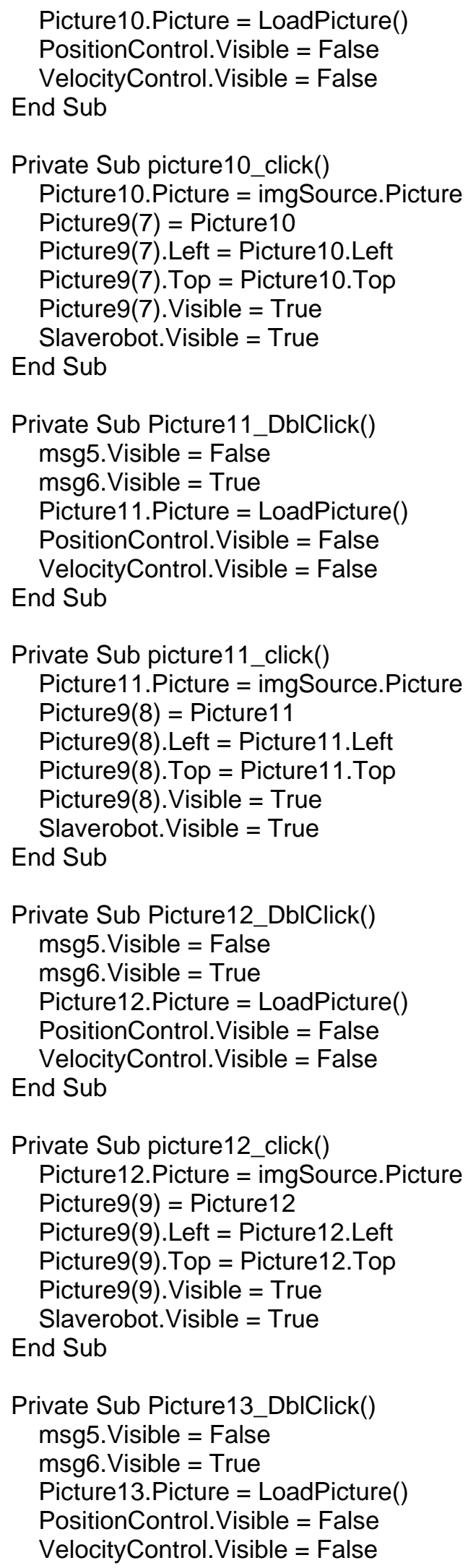




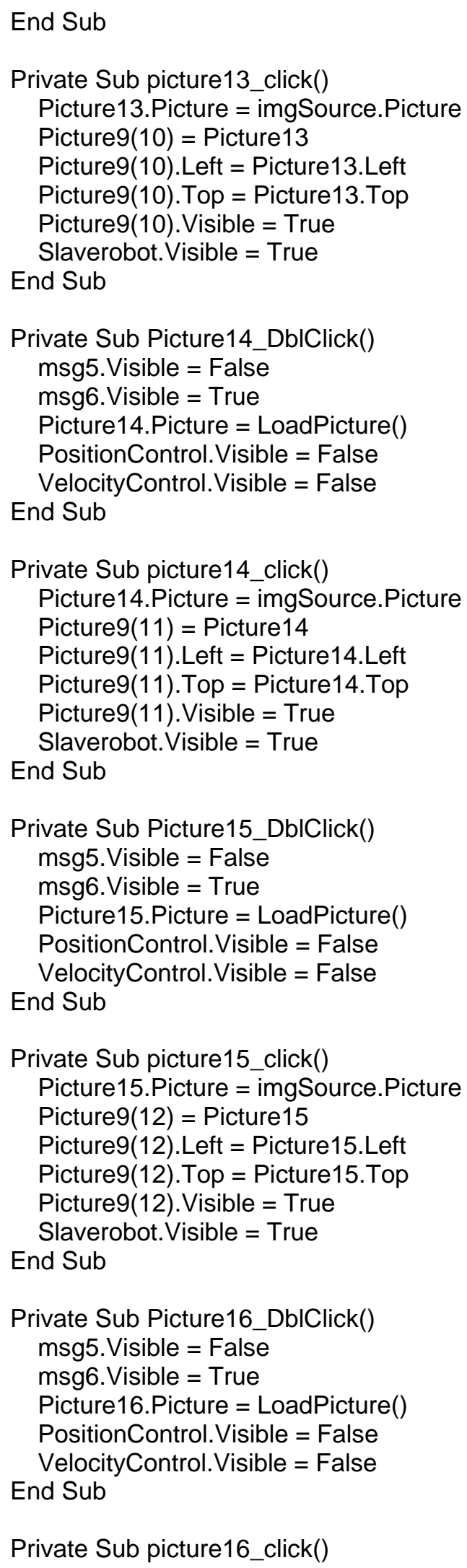




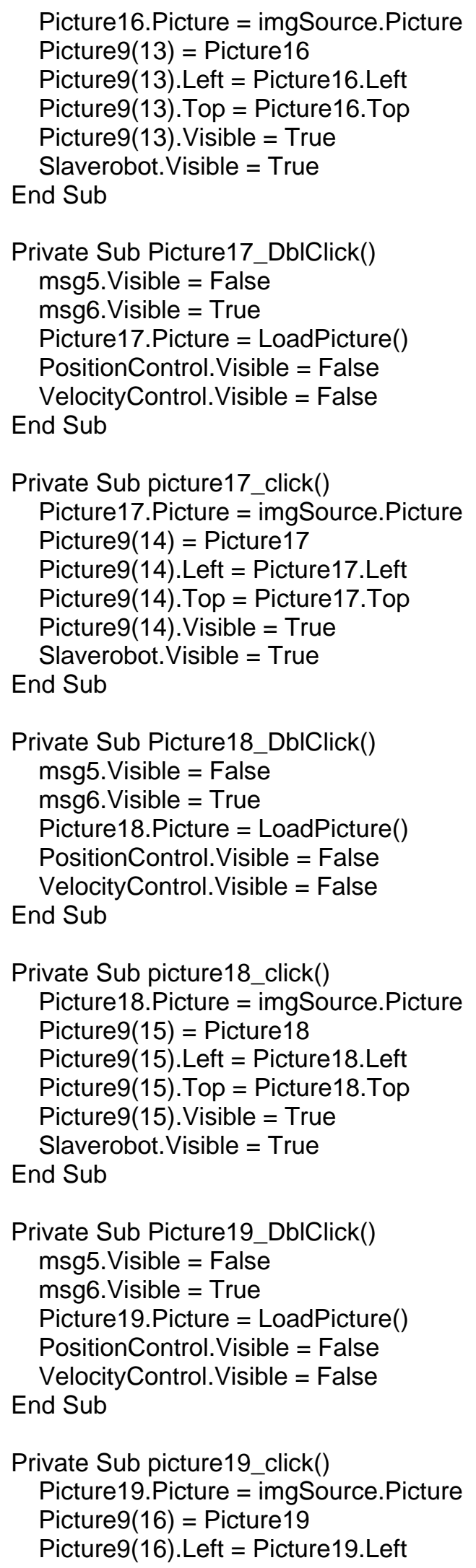




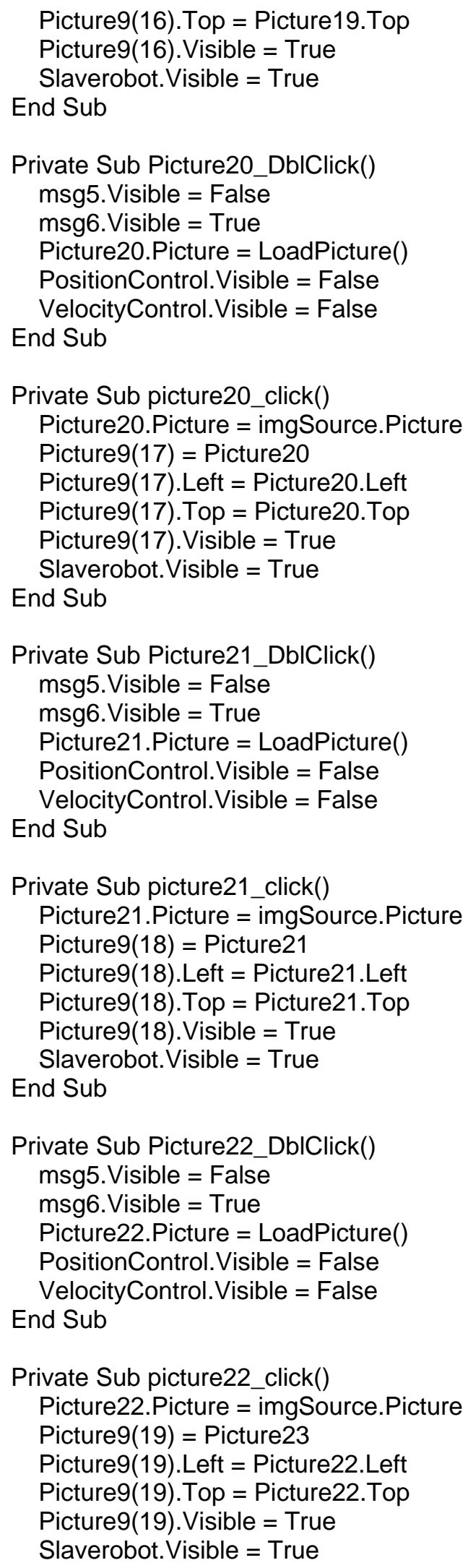




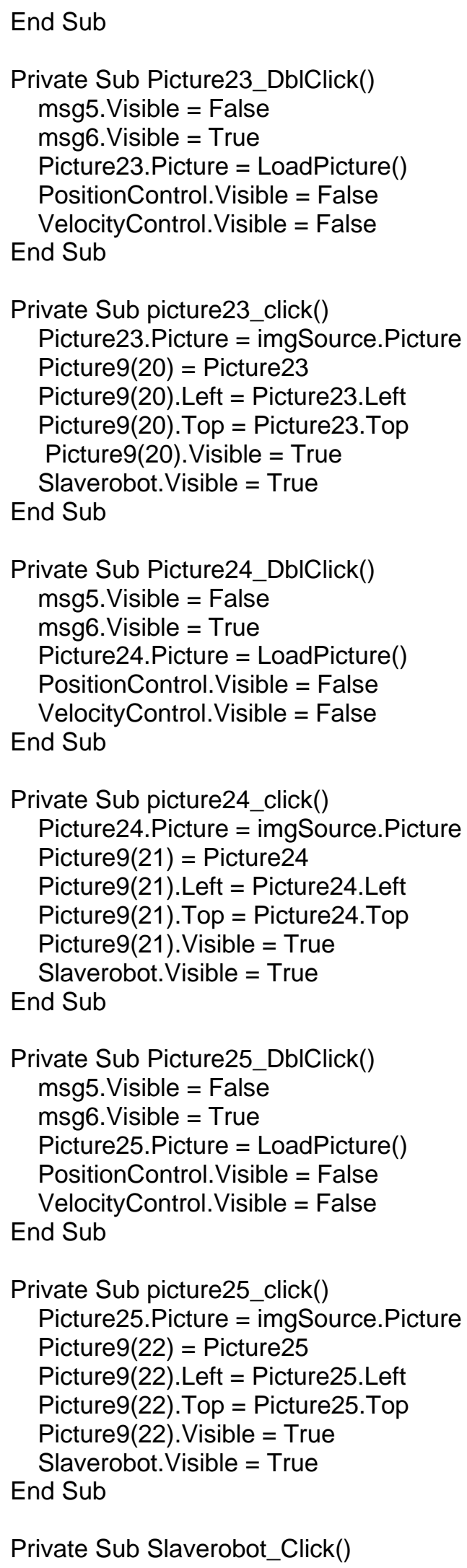

Private Sub Slaverobot_Click() 


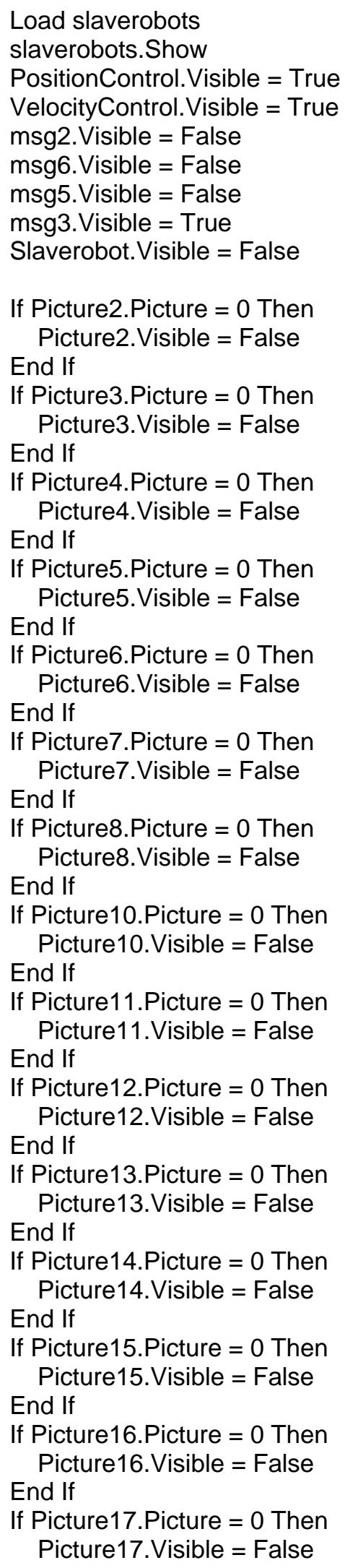




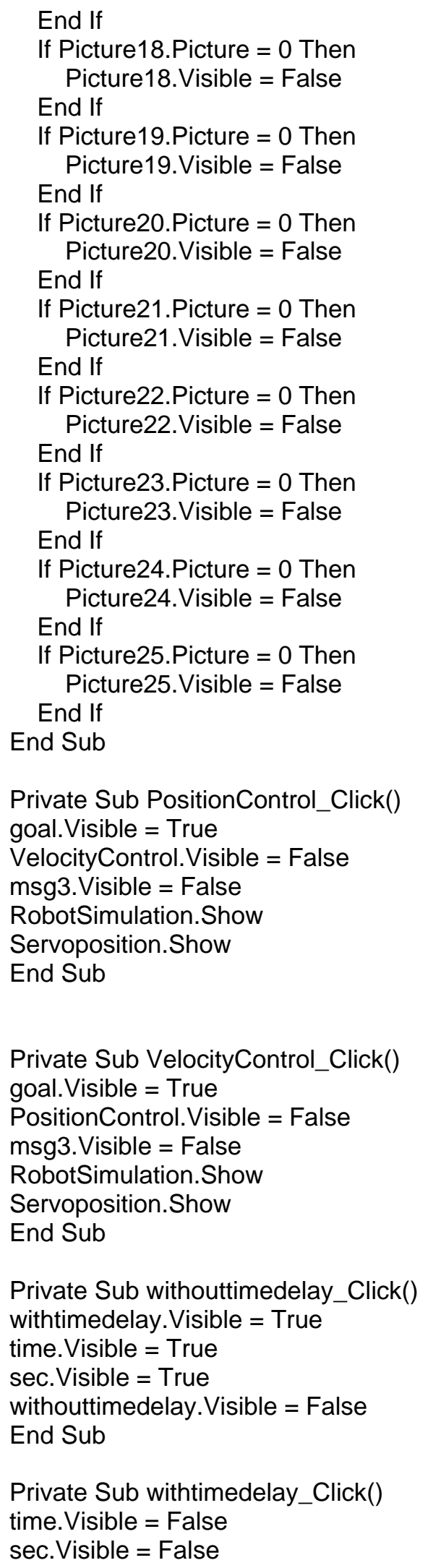




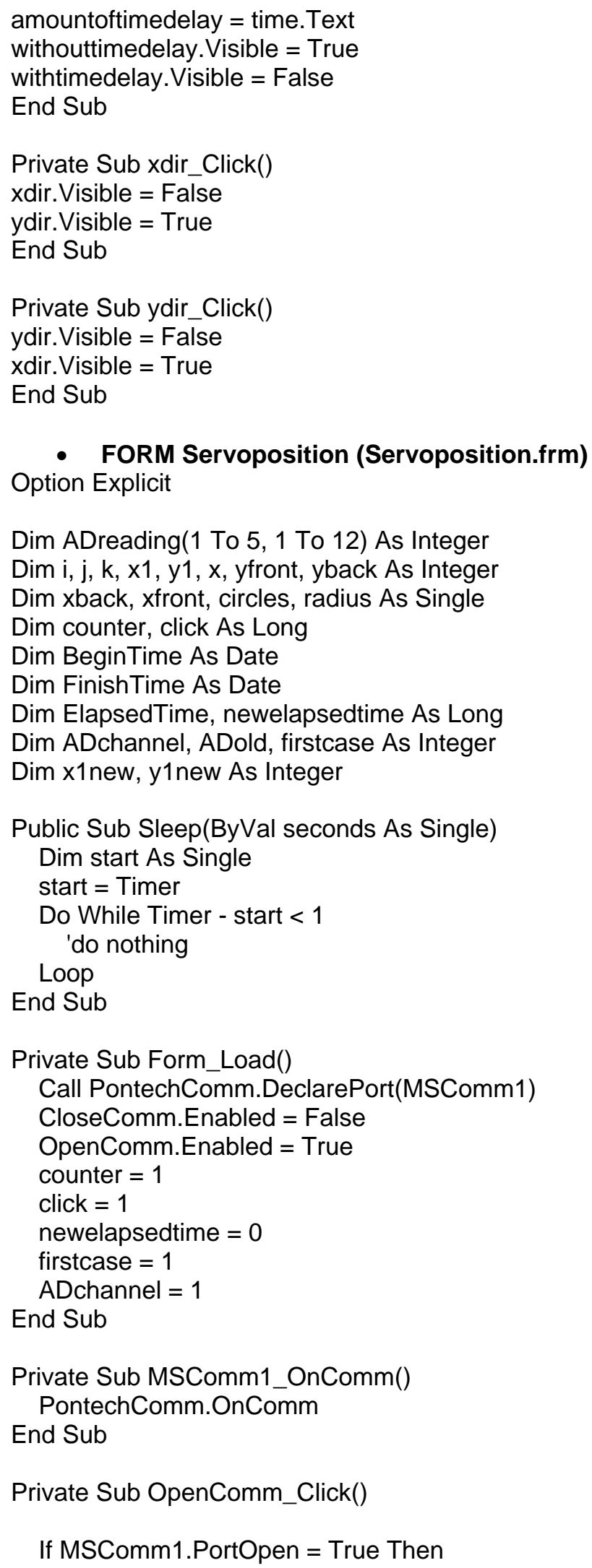




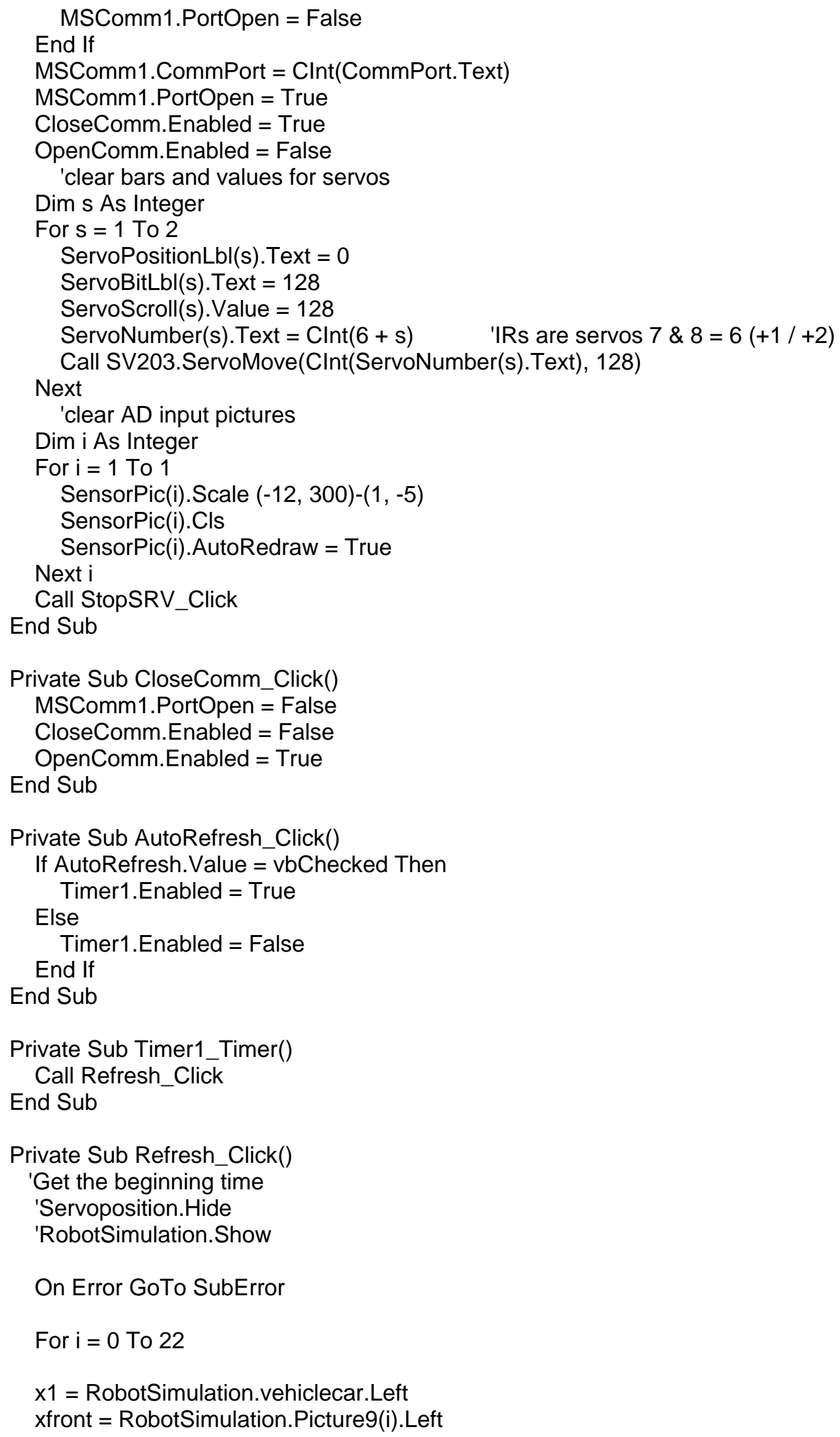




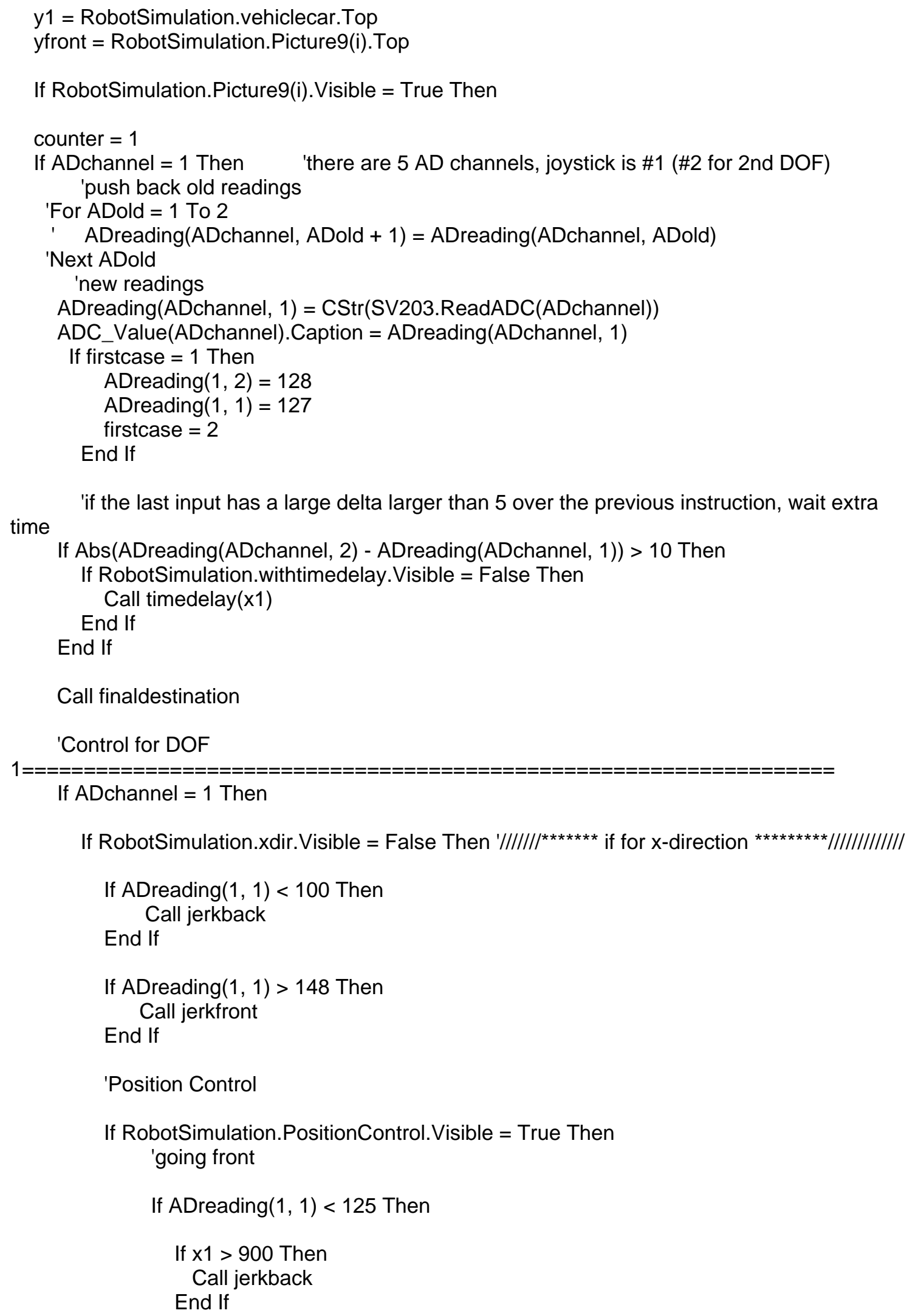

Call finaldestination

'Control for DOF 
Call draw_circles_front

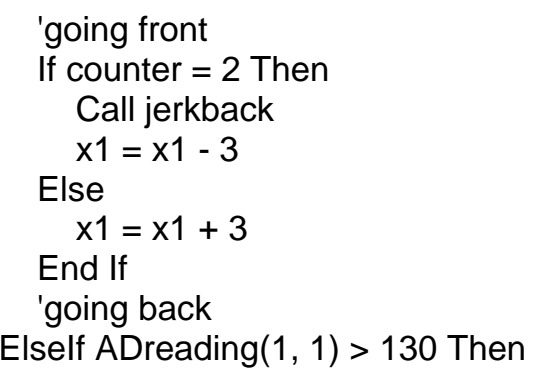

Call draw_circles_back

If $x 1<50$ Then

Call jerkfront

End If

If counter $=2$ Then

Call jerkfront

$x 1=x 1+3$

Else

$x 1=x 1-3$

End If

End If ' ${ }^{1 * \star \star \star \star \star \star \star \star \star \star \star \star \star \star ~ E n d ~ o f ~ g o i n g ~ f r o n t / b a c k ~}{ }^{\star \star \star \star \star \star \star \star \star ~}$

End If $" * \star \star \star \star \star \star \star \star \star \star \star \star * E$ End Of position Control

' Velocity Control

If RobotSimulation.VelocityControl.Visible $=$ True Then

'going front

If ADreading $(1,1)<125$ Then

If $x 1>900$ Then

Call jerkback

End If

If ADreading $(1,1)<125$ And ADreading( 1,1$)>119$ Then

Call draw_circles_front

If counter $=2$ Then

Call jerkback

$\mathrm{x} 1=\mathrm{x} 1-5$

Else

$x 1=x 1+5$

End If

Elself ADreading $(1,1)<120$ And ADreading $(1,1)>114$ Then 


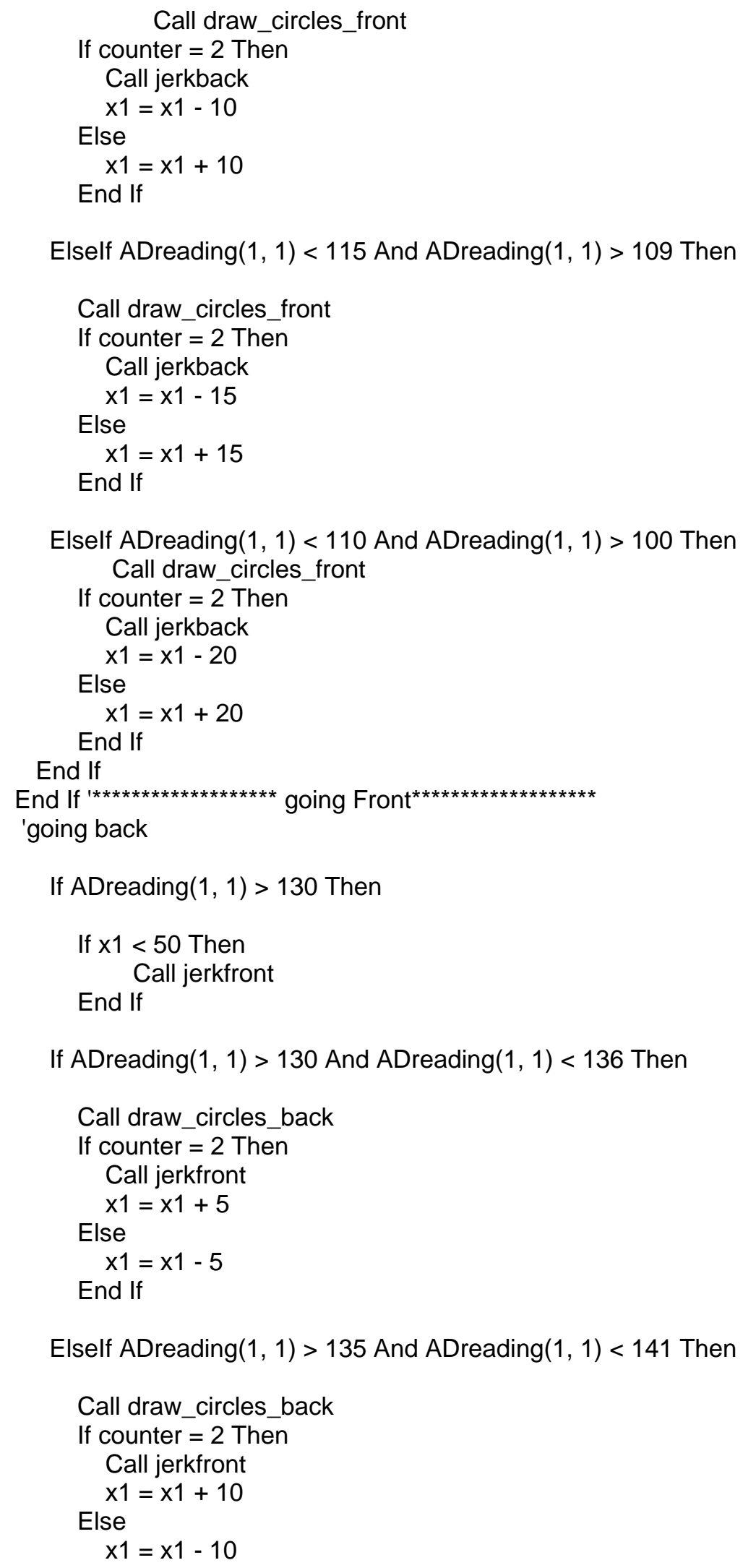


End If

Elself ADreading $(1,1)>140$ And ADreading $(1,1)<146$ Then

Call draw_circles_back

If counter $=2$ Then

Call jerkfront

$\mathrm{x} 1=\mathrm{x} 1+15$

Else

$\mathrm{x} 1=\mathrm{x} 1-15$

End If

Elself ADreading $(1,1)>145$ And ADreading $(1,1)<148$ Then

Call draw_circles_back

If counter $=2$ Then

Call jerkfront

$\mathrm{x} 1=\mathrm{x} 1+20$

Else

$x 1=x 1-20$

End If

End If

End If ${ }^{\prime * \star \star \star \star \star \star \star \star \star \star \star \star \star \star \star \star ~ C l o s e ~ g o i n g ~ b a c k ~}{ }^{\star \star \star \star \star \star \star \star \star \star \star \star \star \star \star \star \star \star ~}$

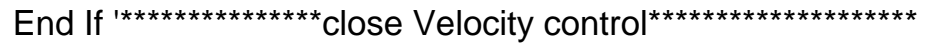

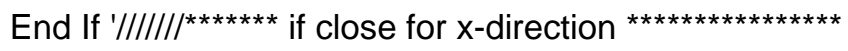

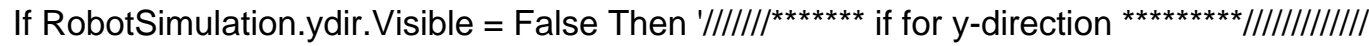

If ADreading $(1,1)<100$ Then

End If

Call jerkfront

If ADreading $(1,1)>148$ Then

Call jerkback

End If

'Position Control

If RobotSimulation.PositionControl. Visible $=$ True Then

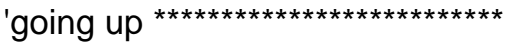

If $A$ Dreading $(1,1)<125$ Then

If $\mathrm{y} 1>680$ Then

Call jerkback

End If

Call draw_circles_up

'going up

If counter $=2$ Then

Call jerkback

$\mathrm{y} 1=\mathrm{y} 1-3$

Else

$y 1=y 1+3$

End If 


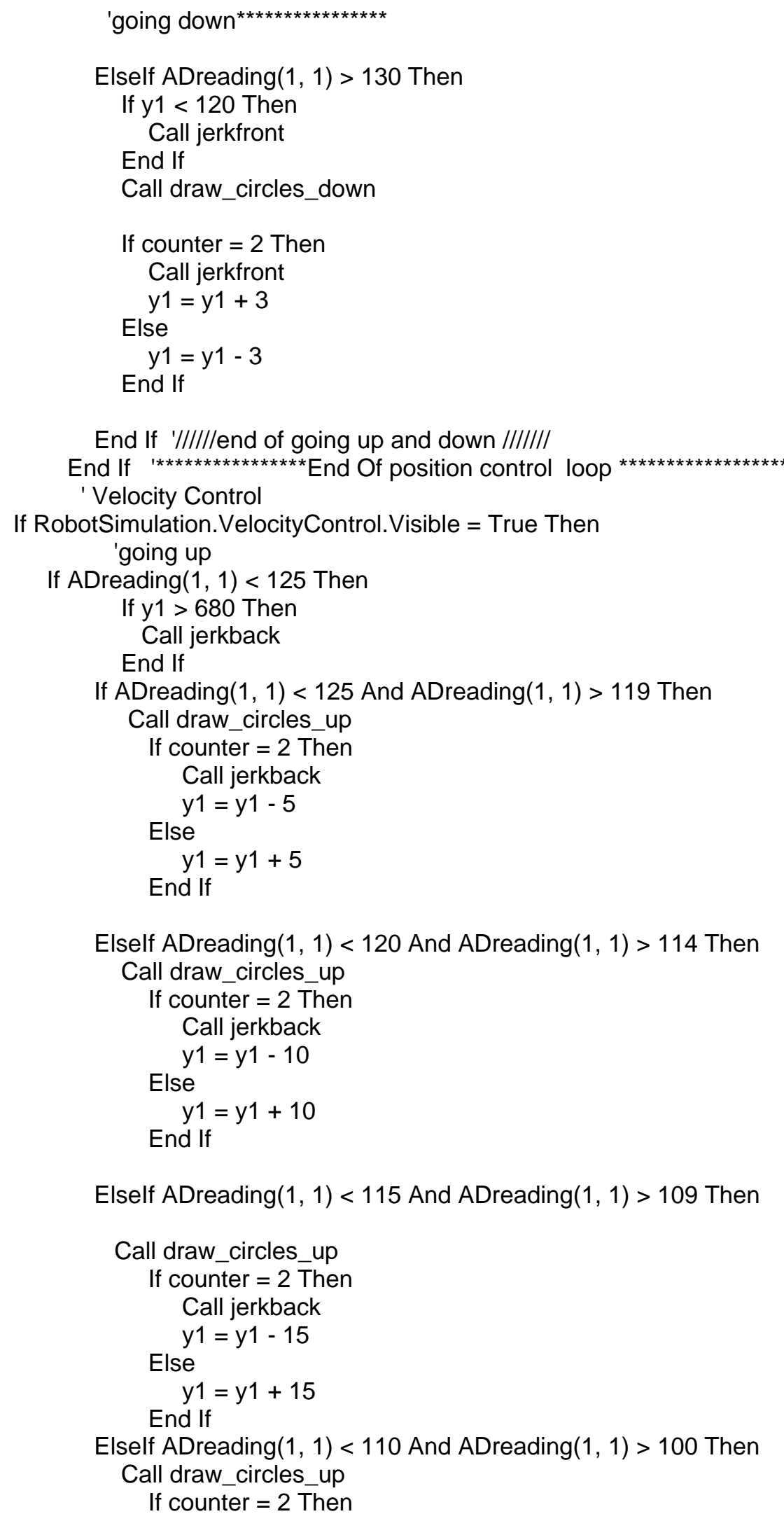

End If '/III//end of going up and down IIIIIII

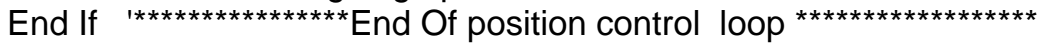

' Velocity Control

If RobotSimulation.VelocityControl. Visible $=$ True Then 'going up

If ADreading $(1,1)<125$ Then

If $\mathrm{y} 1>680$ Then

Call jerkback

End If

If ADreading $(1,1)<125$ And ADreading( 1,1$)>119$ Then

Call draw_circles_up

If counter $=2$ Then

Call jerkback

$\mathrm{y} 1=\mathrm{y} 1-5$

Else

$\mathrm{y} 1=\mathrm{y} 1+5$

End If

Elself ADreading $(1,1)<115$ And ADreading(1, 1) $>109$ Then 


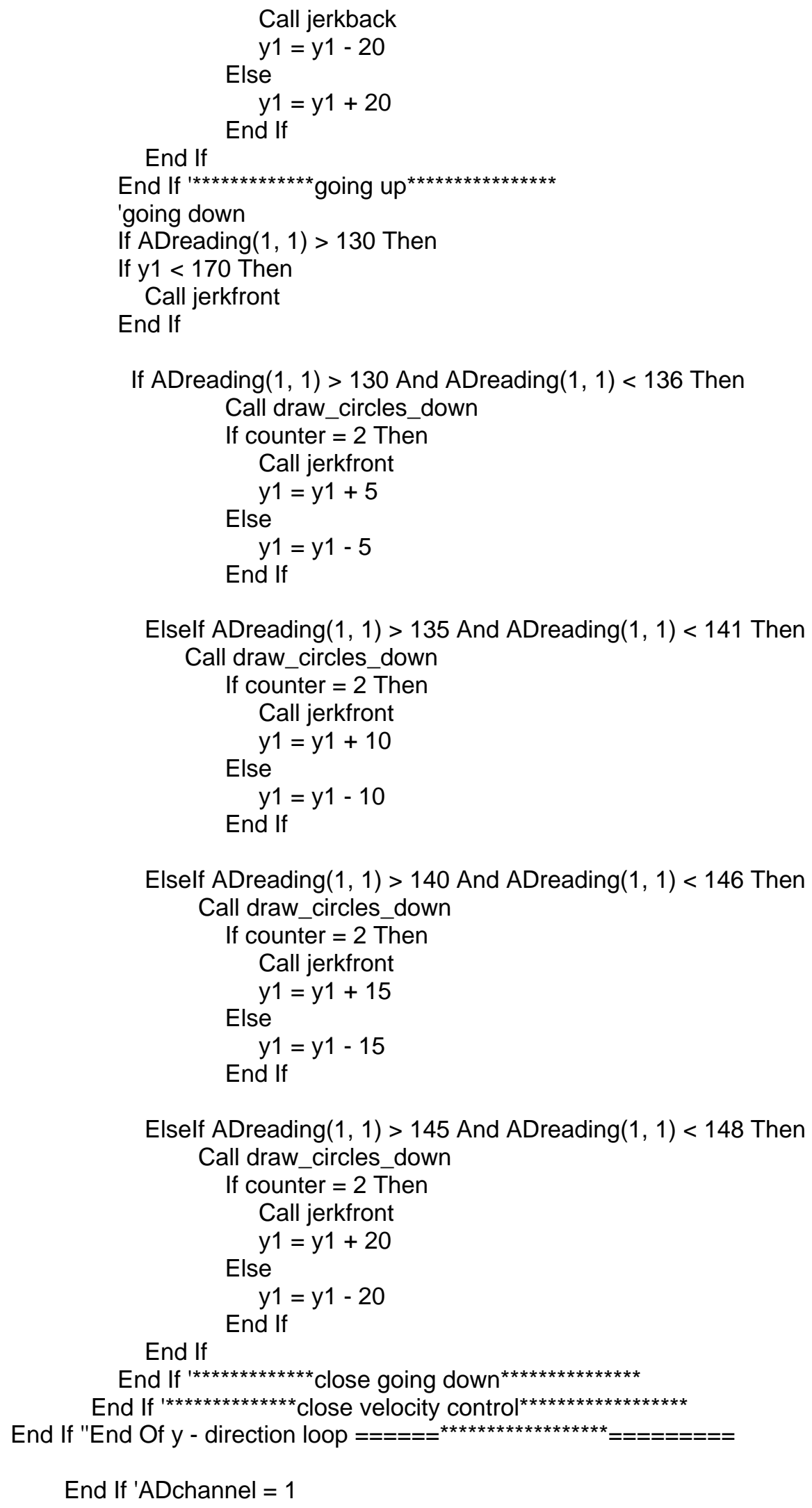

Elself ADreading $(1,1)>145$ And ADreading $(1,1)<148$ Then

Call draw_circles_down

If counter $=2$ Then Call jerkfront 


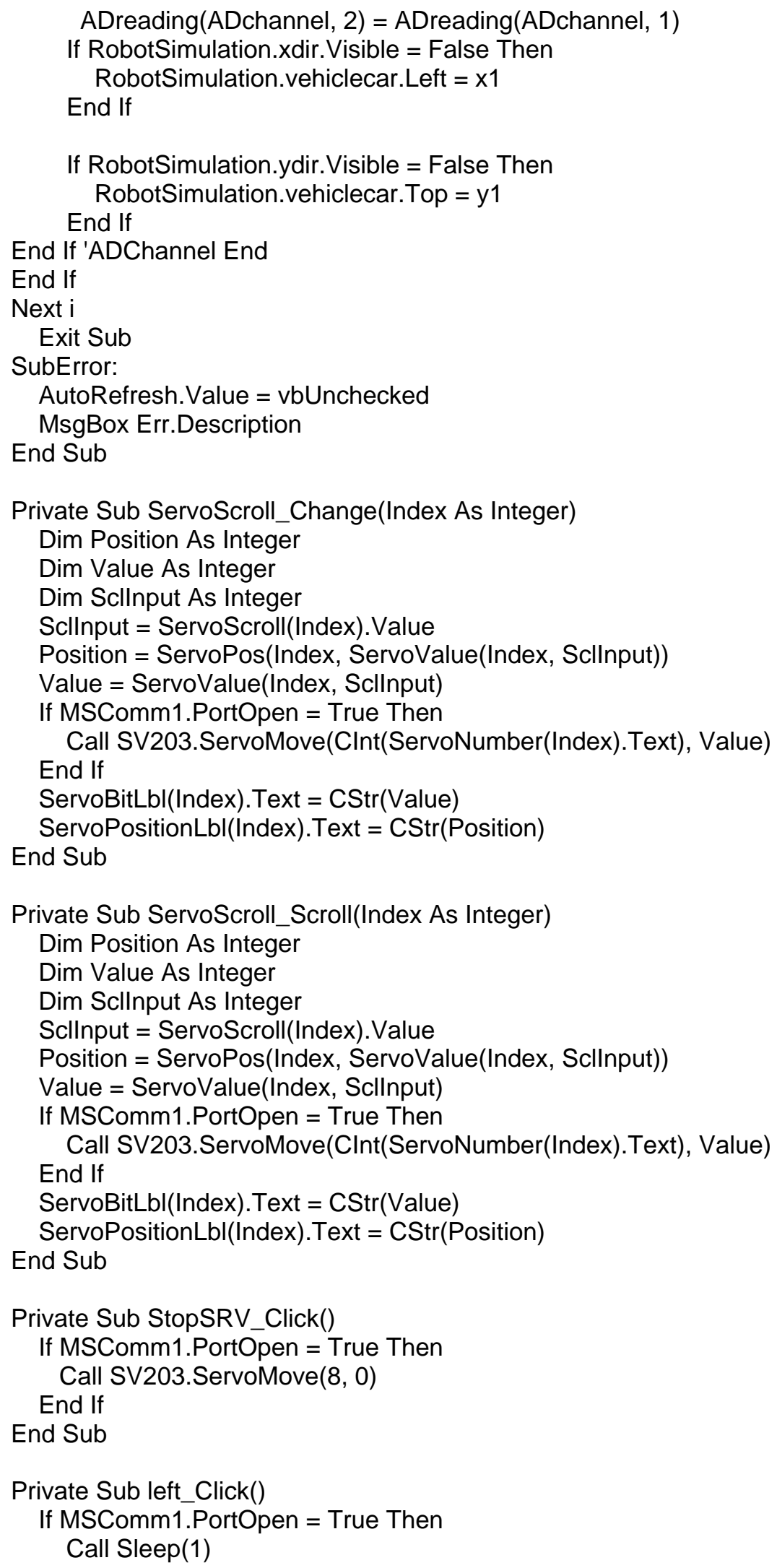




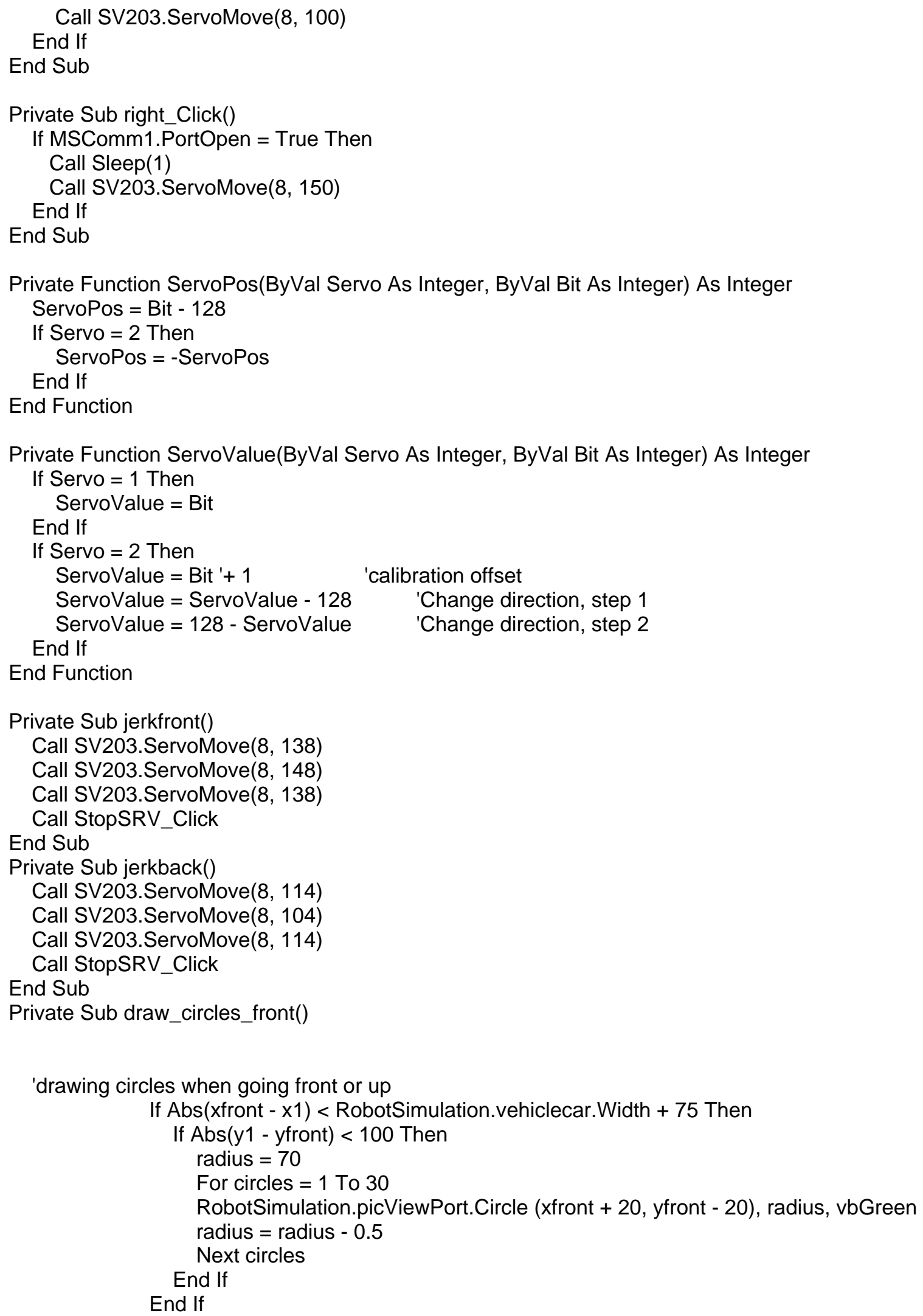




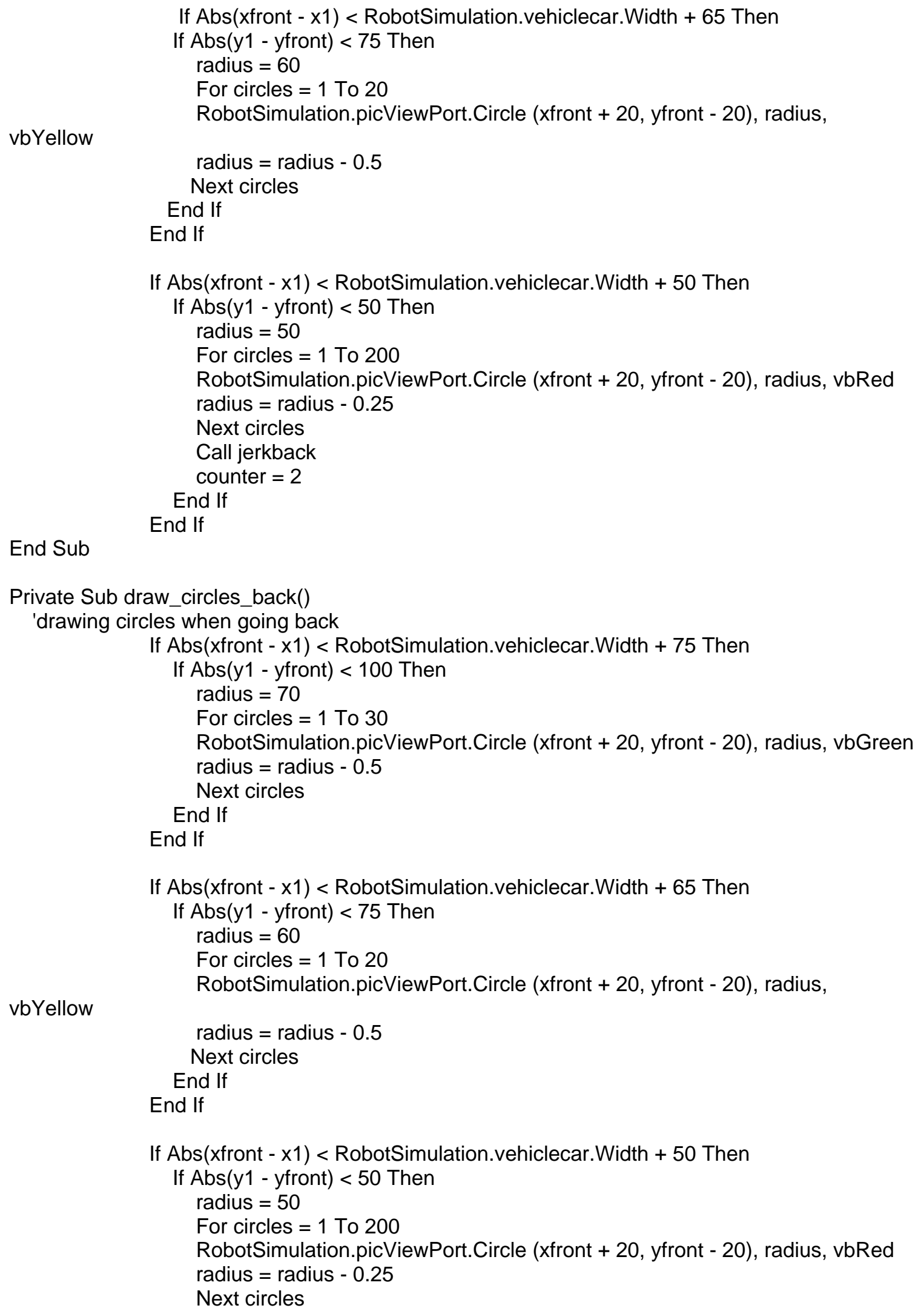




$$
\begin{gathered}
\text { Call jerkfront } \\
\text { counter }=2 \\
\text { End If } \\
\text { End If }
\end{gathered}
$$

Private Sub draw_circles_down()

'drawing circles when going down

If Abs(xfront - x1) < RobotSimulation.vehiclecar.Width +75 Then

If $\mathrm{Abs}(\mathrm{y} 1$ - yfront $)<100$ Then

radius $=70$

For circles $=1$ To 30

RobotSimulation.picViewPort.Circle (xfront +20 , yfront -20 ), radius,

vbGreen

radius $=$ radius -0.5

Next circles

End If

End If

If Abs(xfront $-x 1$ ) $<$ RobotSimulation.vehiclecar.Width +65 Then

If Abs(y1 - yfront) $<75$ Then

radius $=60$

For circles $=1$ To 20

vbYellow

RobotSimulation.picViewPort.Circle (xfront +20 , yfront -20 ), radius,

vbRed

radius $=$ radius -0.5

Next circles

End If

End If

If Abs(xfront $-x 1$ ) $<$ RobotSimulation.vehiclecar.Width +50 Then

If Abs(y1 - yfront) $<50$ Then

radius $=50$

For circles $=1$ To 200

RobotSimulation.picViewPort.Circle (xfront +20 , yfront -20 ), radius,

End Sub

radius $=$ radius -0.25

Next circles

Call jerkfront

counter $=2$

$$
\text { End If }
$$

End If

Private Sub draw_circles_up()

'drawing circles when going down

If Abs(xfront - x1) < RobotSimulation.vehiclecar.Width +75 Then

If $\mathrm{Abs}(\mathrm{y} 1-\mathrm{yfront})<100$ Then

radius $=70$

For circles $=1$ To 30 
vbGreen

RobotSimulation.picViewPort.Circle (xfront +20 , yfront -20 ), radius,

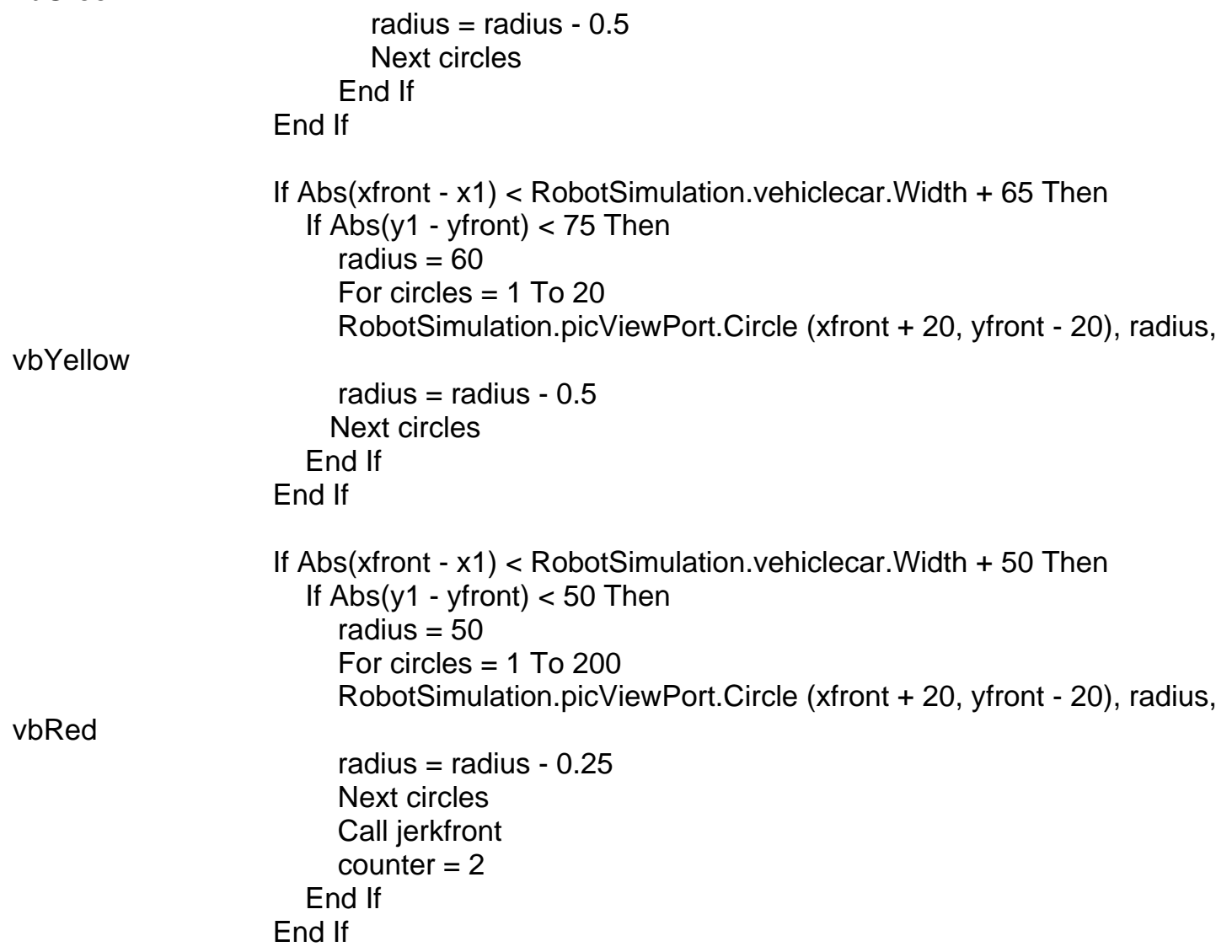

Private Sub timedelay(x1new)

Servoposition. Hide

RobotSimulation.Show

'Timer1. Enabled $=$ False

Timer2. Enabled $=$ True

Dim newtime As Integer

newtime $=0$

For $\mathrm{i}=0$ To 22

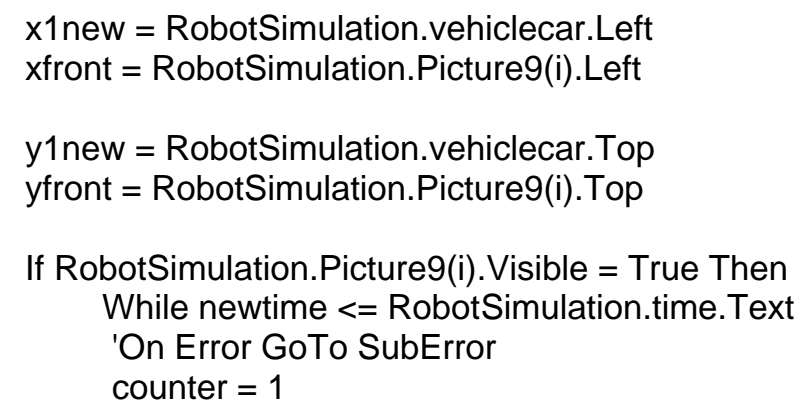


'Control for DOF

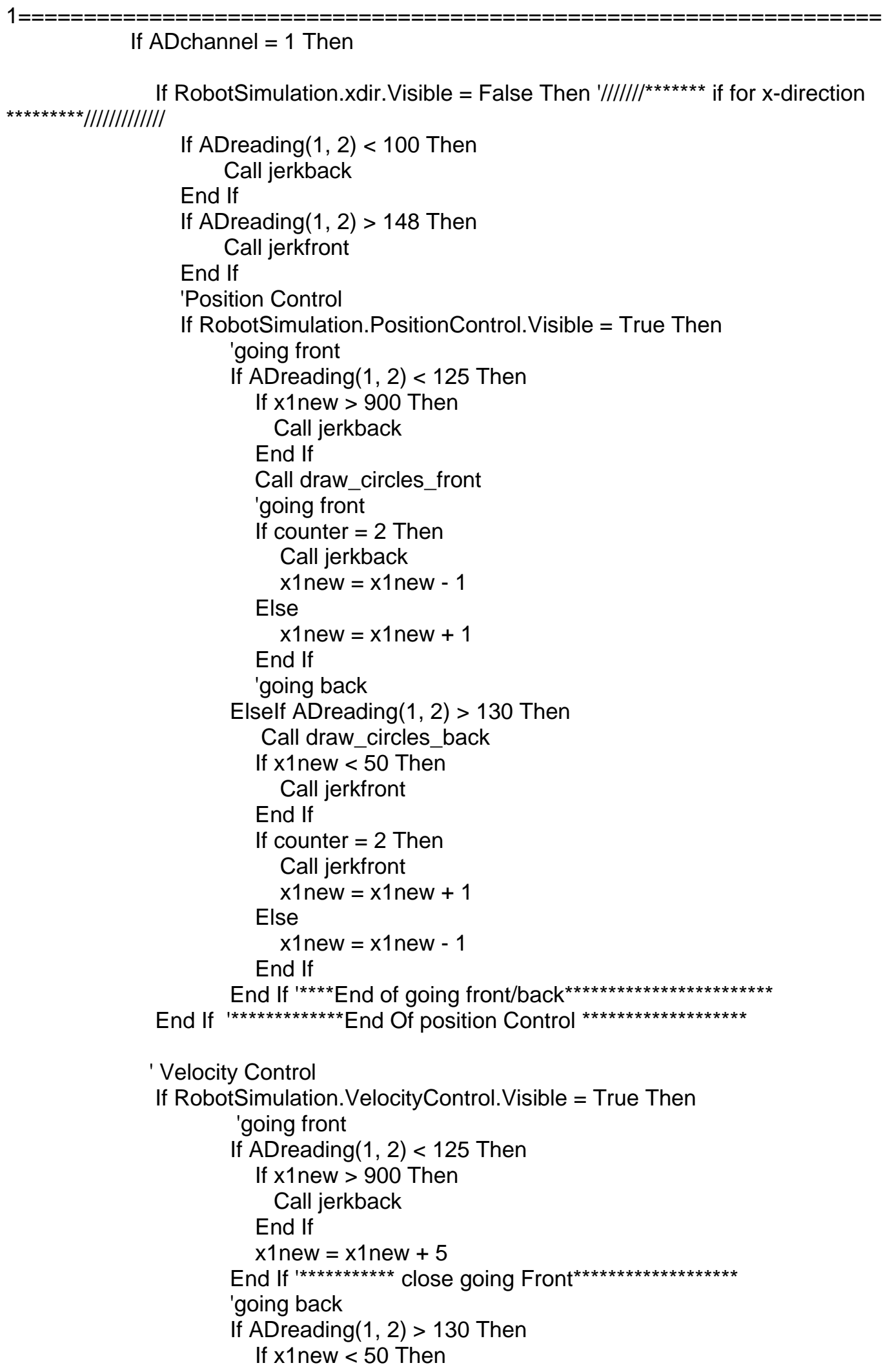




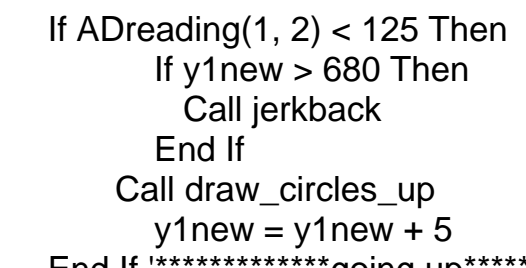

End If ${ }^{\prime \star \star \star \star \star \star \star \star \star \star \star \star \star \star \star ~}$ going up up

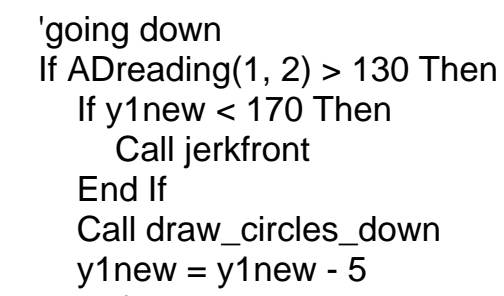

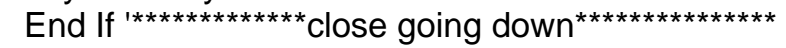

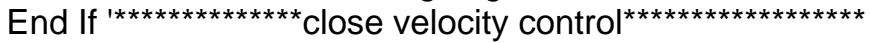

End If "End Of $\mathrm{y}$ - direction loop ======

End If 'ADchannel = 1

'draw readings

'SensorPic(ADchannel).Cls

If RobotSimulation.$x d i r$. Visible $=$ False Then

Sleep 0.000001

RobotSimulation.vehiclecar. Left $=x 1 \mathrm{new}$

End If

If RobotSimulation.ydir. Visible $=$ False Then

Sleep 0.000001

RobotSimulation.vehiclecar.Top = y1 new

$$
\text { End If }
$$

newtime $=$ newtime + Timer2.Interval

'Debug.Print newtime

Wend

End If

Next $\mathrm{i}$

End Sub

Private Sub finaldestination()

If $\operatorname{Abs}(x 1$ - RobotSimulation.goal.Left $)<$ RobotSimulation.goal.Width +10 Then

If Abs(y1 - RobotSimulation.goal.Top) < RobotSimulation.goal.Height +10 Then

RobotSimulation.goal. Visible $=$ False

RobotSimulation.stopsign. Visible $=$ True

End If

Else

RobotSimulation.goal. Visible $=$ True

RobotSimulation. stopsign . Visible $=$ False

End If

End Sub

\section{- FORM Slaverobots (Slaverobot.frm)}

Private Sub black_Click()

Unload Me 
RobotSimulation vehiclecar $=$ RobotSimulation .blackmovingrobot RobotSimulation .selectedrobot $=$ RobotSimulation .vehiclecar

RobotSimulation. robot. Visible $=$ True

RobotSimulation. selectedrobot. Visible $=$ True

RobotSimulation.vehiclecar. Visible $=$ True

End Sub

Private Sub blue_Click()

Unload Me

RobotSimulation.vehiclecar $=$ RobotSimulation . bluewalkingrobot

RobotSimulation. selectedrobot $=$ RobotSimulation.vehiclecar

RobotSimulation.robot.Visible $=$ True

RobotSimulation. selectedrobot. Visible $=$ True

RobotSimulation. vehiclecar. Visible $=$ True

End Sub

Private Sub brown_Click()

Unload Me

RobotSimulation vehiclecar $=$ RobotSimulation .brownwalkingrobot

RobotSimulation. selectedrobot $=$ RobotSimulation $\cdot$ vehiclecar

RobotSimulation.robot. Visible $=$ True

RobotSimulation. selectedrobot. Visible $=$ True

RobotSimulation.vehiclecar.Visible $=$ True

End Sub

Private Sub Car_Click()

Unload Me

RobotSimulation $\cdot$ vehiclecar $=$ RobotSimulation.vehiclecar

RobotSimulation. selectedrobot $=$ RobotSimulation.vehiclecar

RobotSimulation. robot.Visible $=$ True

RobotSimulation. selectedrobot. Visible $=$ True

RobotSimulation. vehiclecar. Visible $=$ True

End Sub

Private Sub dinasour_Click()

Unload Me

RobotSimulation .vehiclecar $=$ RobotSimulation .dinasour

RobotSimulation. selectedrobot $=$ RobotSimulation .vehiclecar

RobotSimulation. .robot. Visible $=$ True

RobotSimulation. selectedrobot. Visible $=$ True

RobotSimulation. vehiclecar. Visible $=$ True

End Sub 


\section{APPENDIX B - VISUAL BASIC 1-DOF FRMC CONTROL PROGRAM FOR TELEOPERATION OVER INTERNET}

We have four modules and five forms for this program on the server side and four modules and two forms on the client side.

We will list all the modules first and then forms on the server side.

\section{SERVER SIDE MODULES}

- MODULE PontechComm (PontechComm.bas)

Code for this module is same as the code listed for Module PontechComm in Appendix A

- MODULE SV203 (SV203.bas)

Code for this module is same as the code listed for Module SV203 in Appendix A

- MODULE Utility_Time (Utility_Time.bas)

Code for this module is same as the code listed for Module Utility_Time in Appendix A

- MODULE STP100 (STP100.bas)

Code for this module is same as the code listed for STP100 in Appendix A

\section{SERVER SIDE FORMS}

- FORM Masterrobot (Masterrobot.frm)

Option Explicit

Public Sub CmdLoad_Click()

Load Robotsimulation

Robotsimulation. Visible $=$ True

End Sub

Sub SendData(data As String)

'Check to see if we're connected to a client

If Winsock.Tag = "CONNECTED" Then

'Send the data

Winsock.SendData data \& vbCrLf

End If

End Sub

Sub Status(data As String)

'Update the status label

IblStatus.Caption = "Status: " \& data

End Sub

Private Sub cmdSend_Click()

'Send the data to the client if it

'is not blank

If txtSend.Text <> "'" Then

SendData txtSend.Text

txtSend. Text = "'"

End If

End Sub 
Private Sub Form_Load()

'Set the caption with your IP

IbllP.Caption = "your ip: " \& Winsock.LocallP

'Listen for incoming connection requests

Winsock.Listen

Status "Awaiting connection.."

End Sub

Private Sub txtData_Change()

'Set the cursor to the last character of the textbox

txtData.SelStart = Len(txtData. Text)

End Sub

Private Sub Winsock_Close()

'Client closed connection, close the Winsock on this side

Winsock.Close

Winsock. Tag = "CLOSED"

'Update status

Status "Connection closed, awaiting new connection.."

'Re-listen for incoming connection requests

Winsock.Listen

End Sub

Private Sub Winsock_ConnectionRequest(ByVal requestID As Long)

'Update status

Status "Accepting connection request"

'Close winsock

Winsock.Close

'Accept the connection request

Winsock.Accept requestID

Winsock.Tag = "CONNECTED"

'Update status

Status "Connected"

End Sub

Private Sub Winsock_DataArrival(ByVal bytesTotal As Long)

Dim Buffer As String

'Update status

Status "Data has arrived"

'Get the data being sent by the client

Winsock.GetData Buffer

'Put incoming data into the Data textbox

txtData = txtData \& "'" \& Buffer

Buffer $=$ UCase (Buffer)

Buffer = Left(Buffer, Len(Buffer) -2$)$

Buffer $=$ Left $($ Buffer, 3 )

txtData. Text $=$ Buffer

Call servoposition.Refresh_Click

'Update the status back to "Connected"

Status "Connected"

End Sub 
- FORM Obstacles (Obstacles.frm)

Code for this form is same as the code listed for Form Obstacles in Appendix A

- FORM Slaverobots (Slaverobots.frm)

Code for this form is same as the code listed for Form Slaverobots in Appendix A

- FORM Robotsimulation (Robotsimulation.frm)

'THIS PROGRAM WILL DEMONSTRATE 1-DOF MOVEMENT OF A ROBOT

'WITH SOME OBSTACLES IN ITS PATH

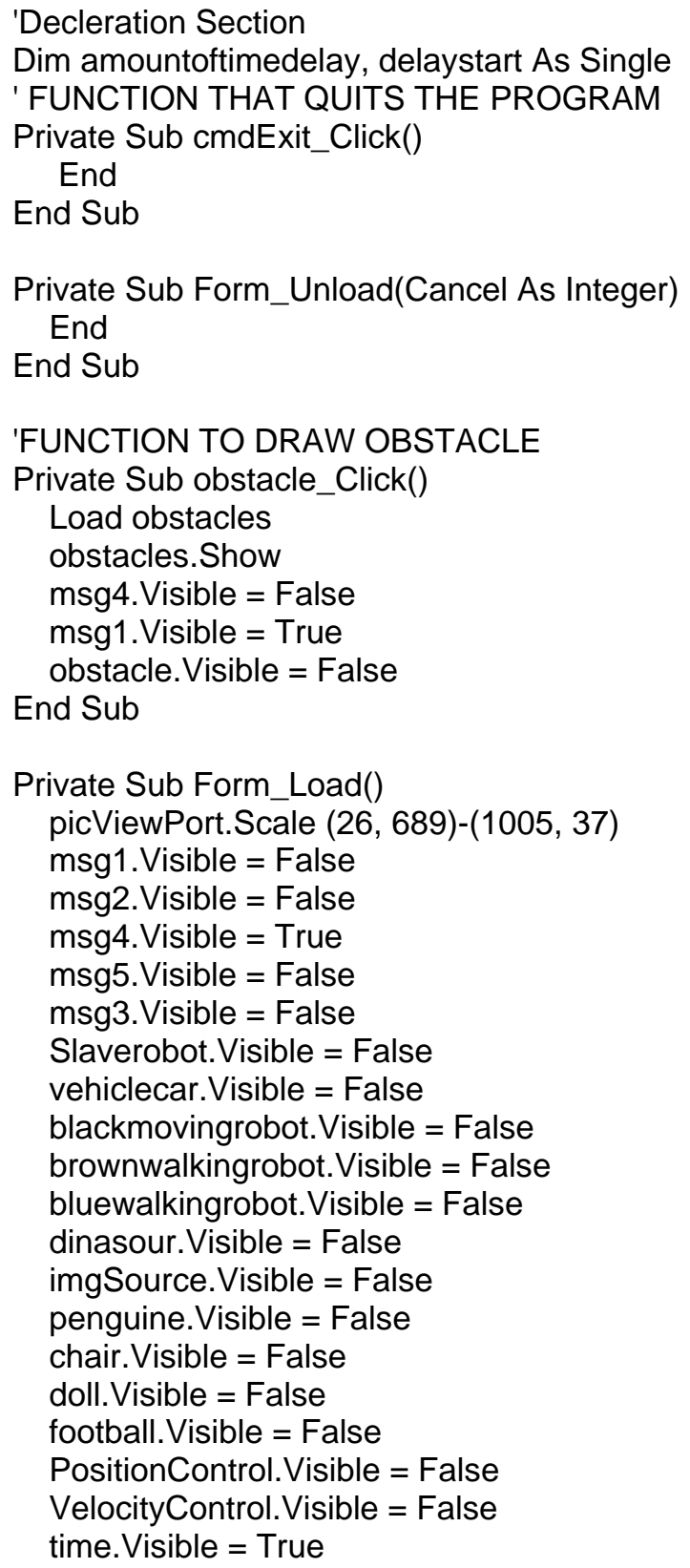




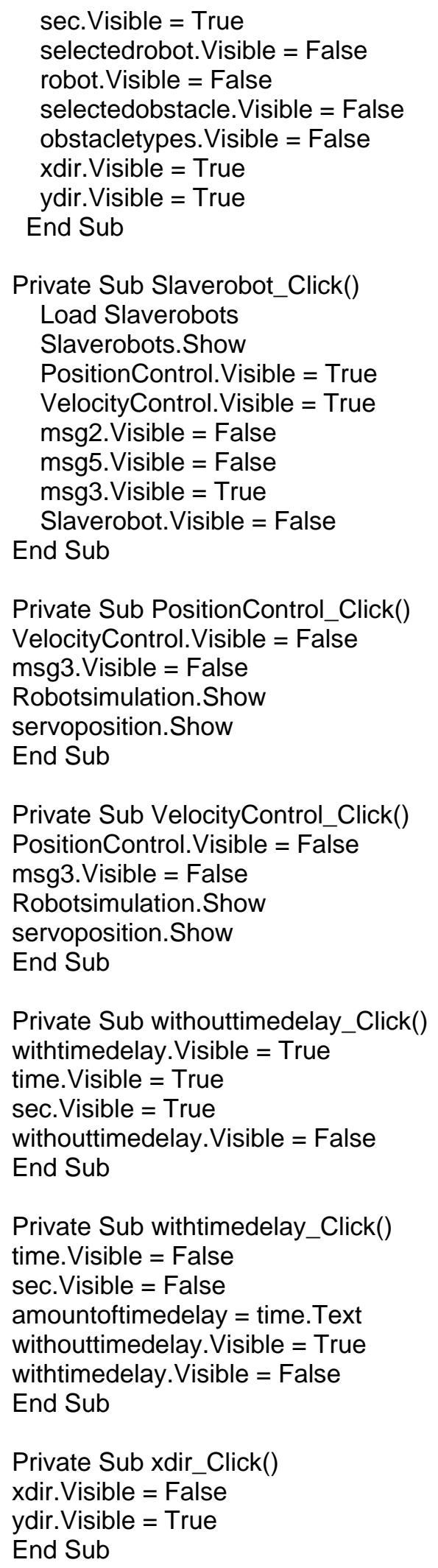


Private Sub ydir_Click()

ydir. Visible $=$ False

xdir. Visible $=$ True

End Sub

- FORM Servoposition (Servoposition.frm)

The whole code of this form is same as the form servoposition in Appendix A except the function refresh_click(). So only function refresh_click has been listed below

Public Sub Refresh_Click()

On Error GoTo SubError

$\mathrm{x} 1=$ Robotsimulation.vehiclecar. Left

counter $=1$

If ADchannel = 1 Then 'there are 5 AD channels, joystick is \#1 (\#2 for 2nd DOF) 'new readings

ADreading(ADchannel, 1$)=$ CStr(SV203.ReadADC(ADchannel))

ADC_Value(ADchannel). Caption $=$ ADreading $($ ADchannel, 1$)$

If firstcase $=1$ Then

ADreading $(1,2)=128$

ADreading $(1,1)=127$

firstcase $=2$

End If

'if the last input has a large delta greater than 10 over the previous instruction, wait extra time

If Abs(ADreading(ADchannel, 2) - ADreading(ADchannel, 1)) > 10 Then

If Robotsimulation. withtimedelay. Visible $=$ False Then

End If

Call timedelay $(x 1)$

End If

'Control for DOF

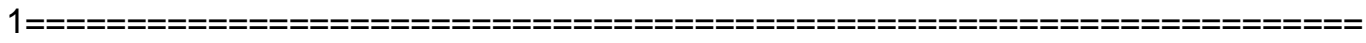

If ADchannel $=1$ Then

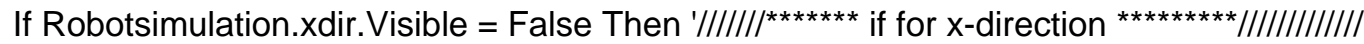

y1 = Robotsimulation.vehiclecar.Top

x1 = Robotsimulation.vehiclecar.Left

If ADreading $(1,1)<100$ Then

End If

Call jerkback

If ADreading $(1,1)>150$ Then

Call jerkfront

End If

'Position Control

If Robotsimulation. PositionControl.Visible $=$ True Then

'going front

If ADreading $(1,1)<125$ Then 


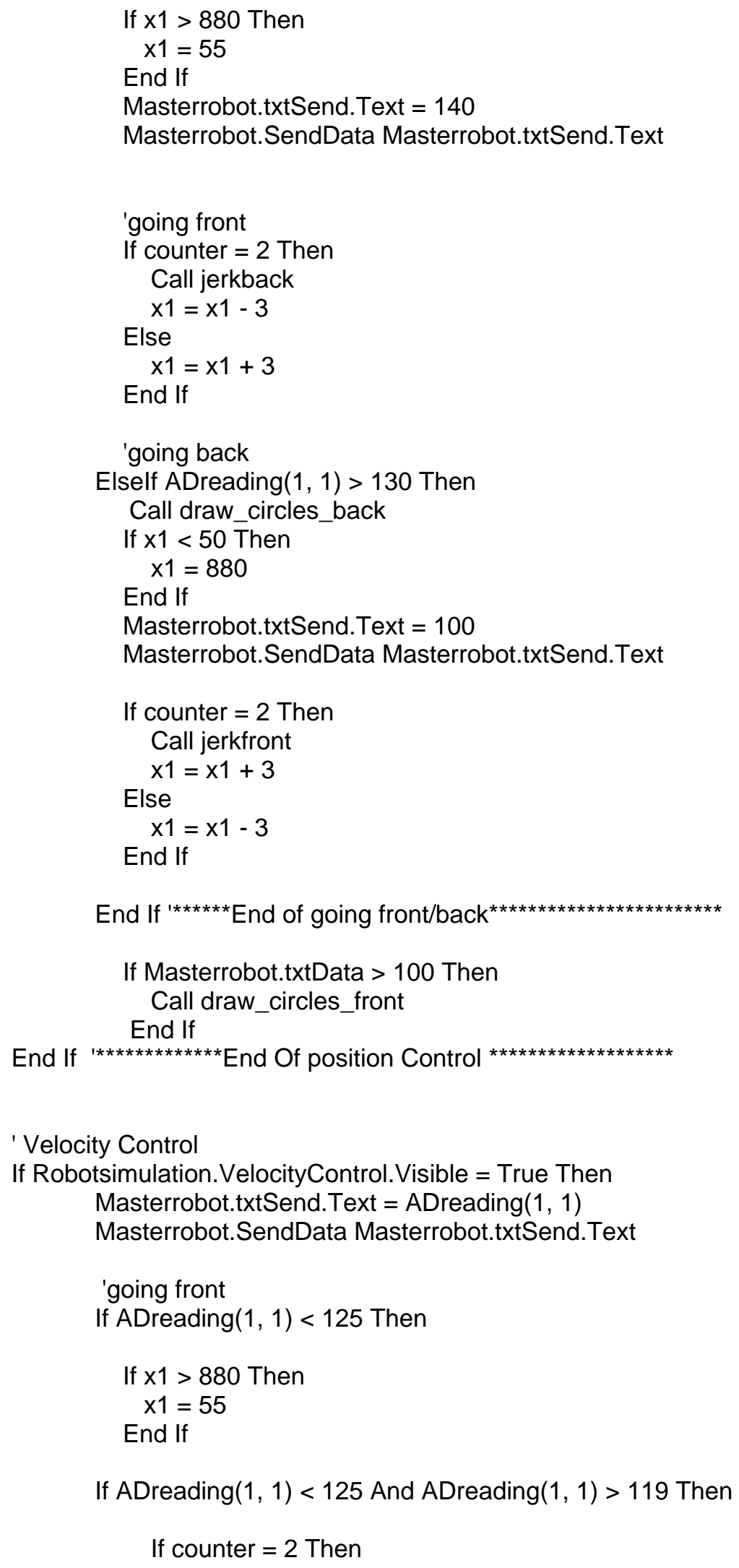

End If ' ${ }^{\star \star \star \star \star \star \star ~ E n d ~ o f ~ g o i n g ~ f r o n t / b a c k ~} \mathrm{~b}^{\star \star \star \star \star \star \star \star \star \star \star \star \star \star \star \star \star \star \star \star \star \star \star \star \star \star \star \star \star ~}$

If Masterrobot.txtData > 100 Then Call draw_circles_front End If

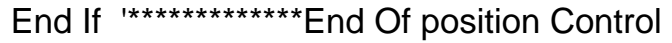




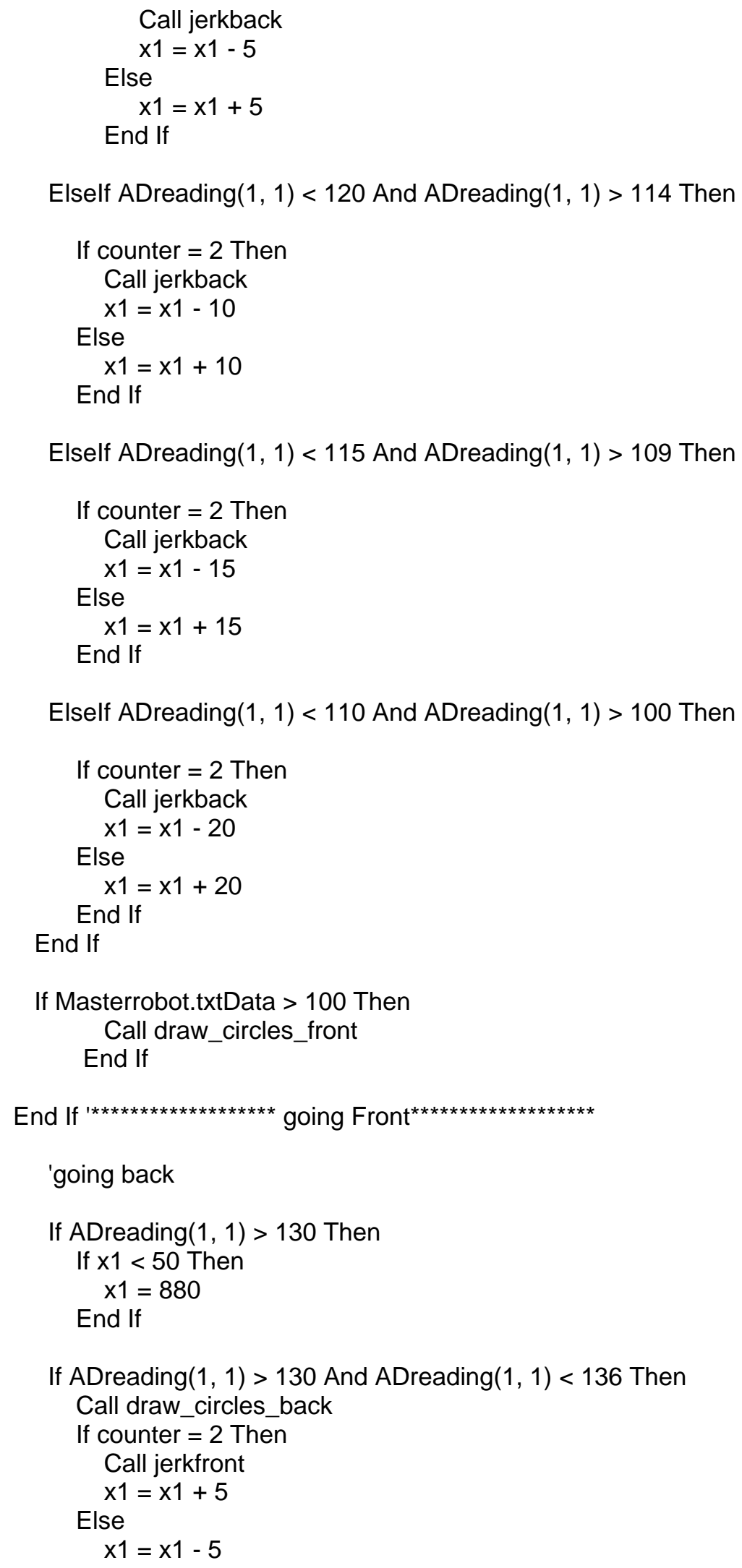


End If

Elself ADreading(1, 1) > 135 And ADreading $(1,1)<141$ Then

Call draw_circles_back

If counter $=2$ Then

Call jerkfront

$\mathrm{x} 1=\mathrm{x} 1+10$

Else

$\mathrm{x} 1=\mathrm{x} 1-10$

End If

Elself ADreading(1, 1) > 140 And ADreading(1, 1) < 146 Then

Call draw_circles_back

If counter $=2$ Then

Call jerkfront

$\mathrm{x} 1=\mathrm{x} 1+15$

Else

$\mathrm{x} 1=\mathrm{x} 1-15$

End If

Elself ADreading $(1,1)>145$ And ADreading $(1,1)<150$ Then

Call draw_circles_back

If counter $=2$ Then

Call jerkfront

$x 1=x 1+20$

Else

$x 1=x 1-20$

End If

End If

If Masterrobot.txtData > 100 Then End If Call draw_circles_front

End If

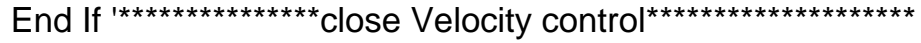

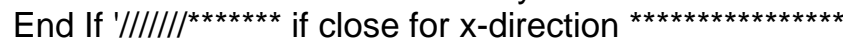

If Robotsimulation.ydir.Visible = False Then "'if for y-direction IIIIIIIIII

$\mathrm{y} 1$ = Robotsimulation.vehiclecar.Top

x1 = Robotsimulation.vehiclecar.Left

If ADreading $(1,1)<100$ Then

Call jerkfront

End If

If ADreading $(1,1)>150$ Then

End If

Call jerkback

'Position Control

If Robotsimulation. PositionControl.Visible $=$ True Then

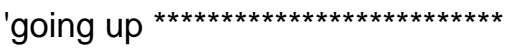




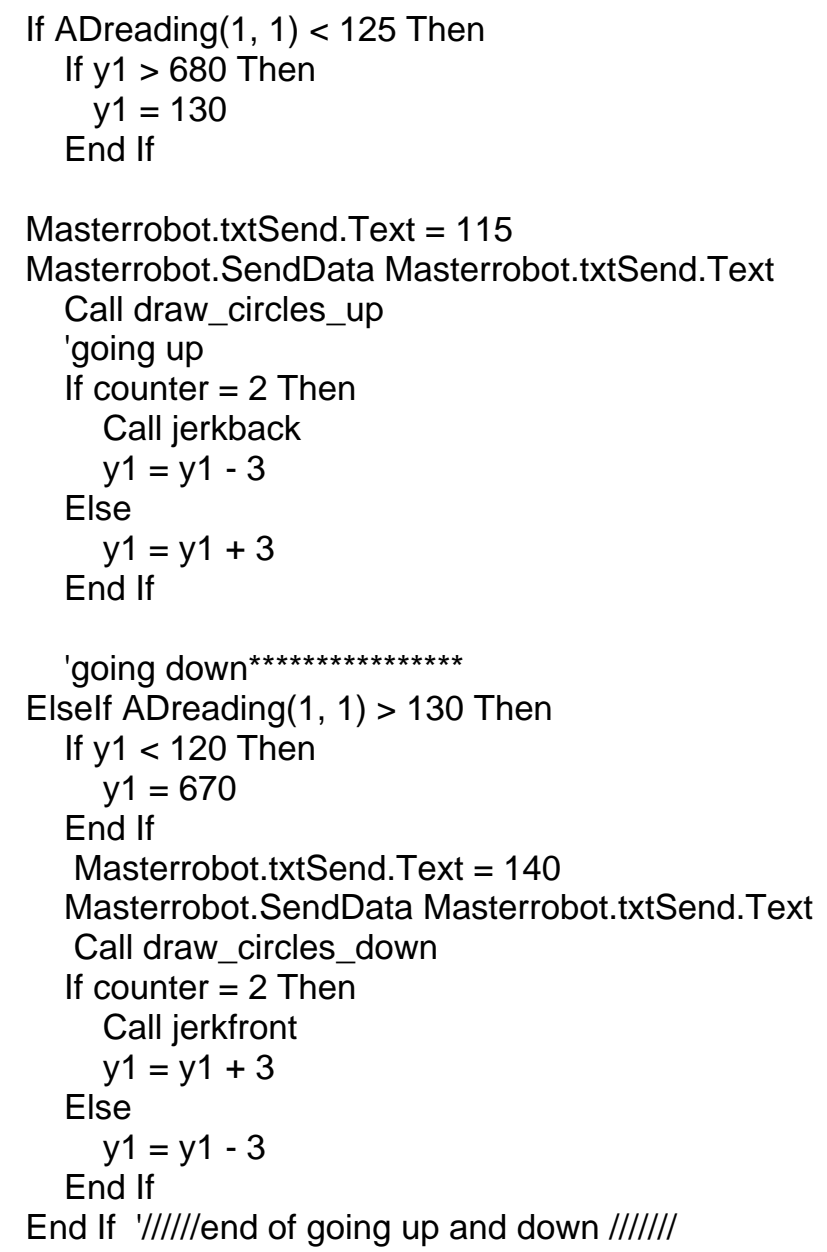

End If ${ }^{\star * \star \star \star \star \star * \star \star \star}$ End Of position control loop

' Velocity Control

If Robotsimulation. VelocityControl. Visible $=$ True Then

Masterrobot.txtSend.Text $=$ ADreading $(1,1)$

Masterrobot.SendData Masterrobot.txtSend.Text

'going up

If ADreading $(1,1)<125$ Then

If $\mathrm{y} 1>680$ Then

$\mathrm{y} 1=130$

End If

If ADreading $(1,1)<125$ And ADreading $(1,1)>119$ Then

Call draw_circles_up

If counter $=2$ Then

Call jerkback

$\mathrm{y} 1=\mathrm{y} 1-5$

Else

$\mathrm{y} 1=\mathrm{y} 1+5$

End If 


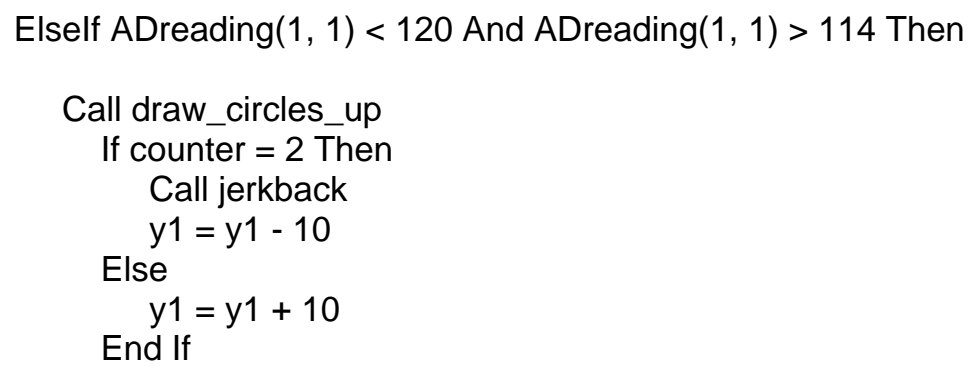

Elself ADreading $(1,1)<115$ And ADreading(1, 1) $>109$ Then

Call draw_circles_up

If counter $=2$ Then

Call jerkback

$\mathrm{y} 1=\mathrm{y} 1-15$

Else

$\mathrm{y} 1=\mathrm{y} 1+15$

End If

Elself ADreading $(1,1)<110$ And ADreading $(1,1)>100$ Then

Call draw_circles_up

If counter $=2$ Then

Call jerkback

$\mathrm{y} 1=\mathrm{y} 1-20$

Else

$\mathrm{y} 1=\mathrm{y} 1+20$

End If

End If

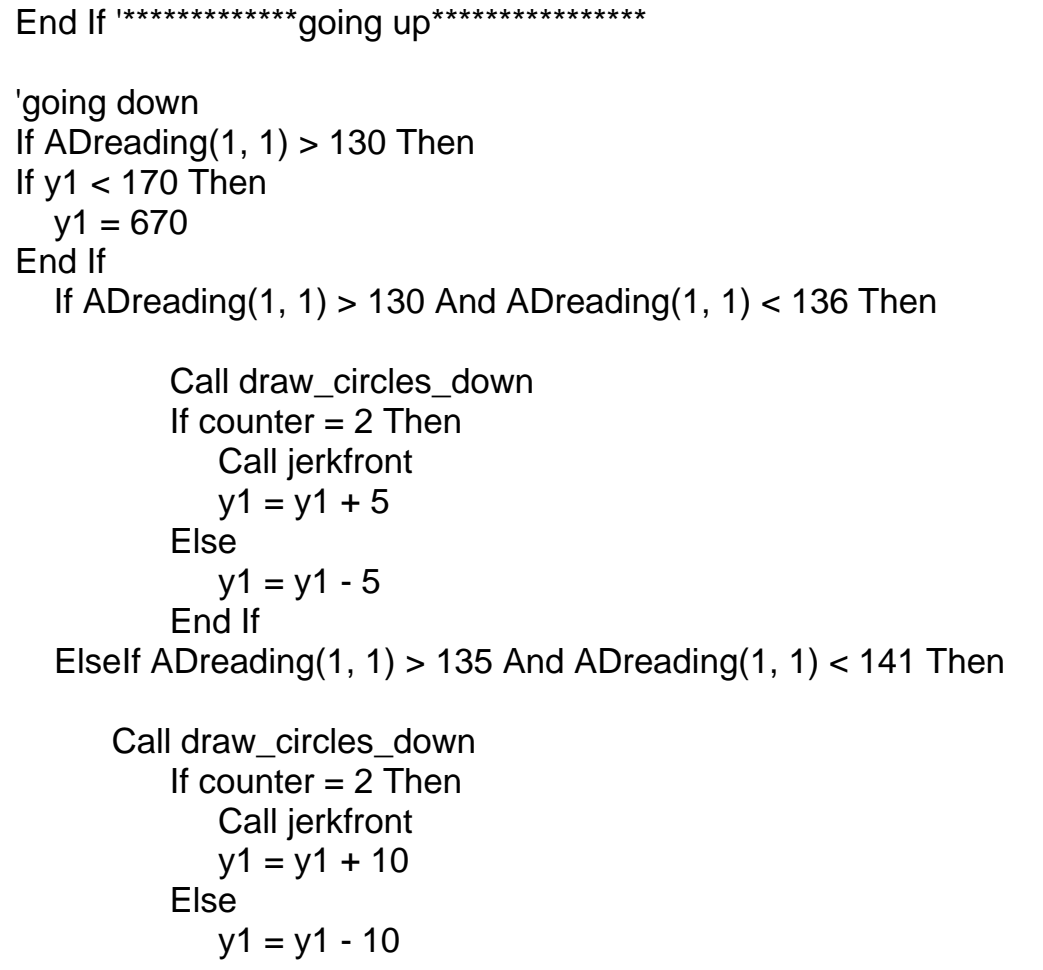


End If

Elself ADreading( 1,1$)>140$ And ADreading $(1,1)<146$ Then

Call draw_circles_down

If counter $=2$ Then

Call jerkfront

$\mathrm{y} 1=\mathrm{y} 1+15$

Else

$\mathrm{y} 1=\mathrm{y} 1-15$

End If

Elself ADreading $(1,1)>145$ And ADreading $(1,1)<150$ Then

Call draw_circles_down

If counter $=2$ Then

Call jerkfront

$\mathrm{y} 1=\mathrm{y} 1+20$

Else

$\mathrm{y} 1=\mathrm{y} 1-20$

End If

End If

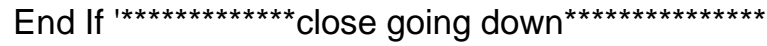

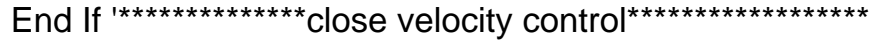

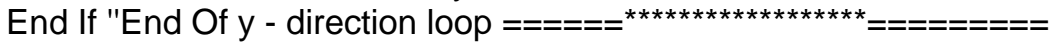

End If 'ADchannel $=1$

ADreading (ADchannel, 2$)=$ ADreading (ADchannel, 1$)$

If Robotsimulation.$x d i r$.Visible $=$ False Then

Robotsimulation.vehiclecar. Left $=x 1$

End If

If Robotsimulation.ydir. Visible $=$ False Then End If

Robotsimulation.vehiclecar. Top $=\mathrm{y} 1$

End If 'ADChannel End

Exit Sub

SubError:

AutoRefresh.Value $=$ vbUnchecked

MsgBox Err.Description

End Sub

\section{CLIENT SIDE MODULES}

- MODULE PontechComm (PontechComm.bas)

Code for this module is same as the code listed for Module PontechComm in Appendix A

- MODULE SV203 (SV203.bas)

Code for this module is same as the code listed for Module SV203 in Appendix A

- MODULE Utility_Time (Utility_Time.bas)

Code for this module is same as the code listed for Module Utility_Time in Appendix A 
- MODULE STP100 (STP100.bas)

Code for this module is same as the code listed for STP100 in Appendix A

\section{CLIENT SIDE FORMS}

\section{- FORM Slaverobot (Slaverobot.frm)}

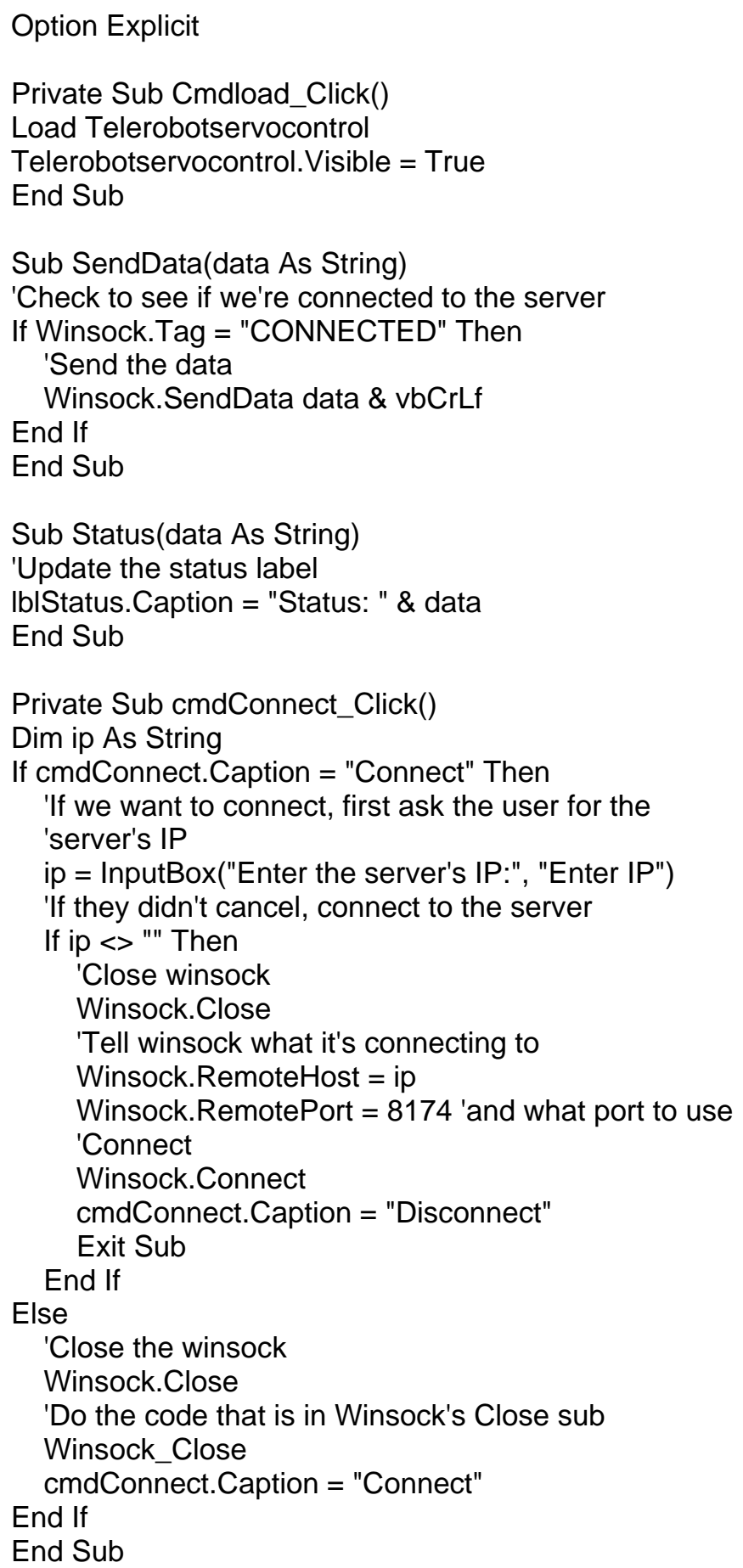




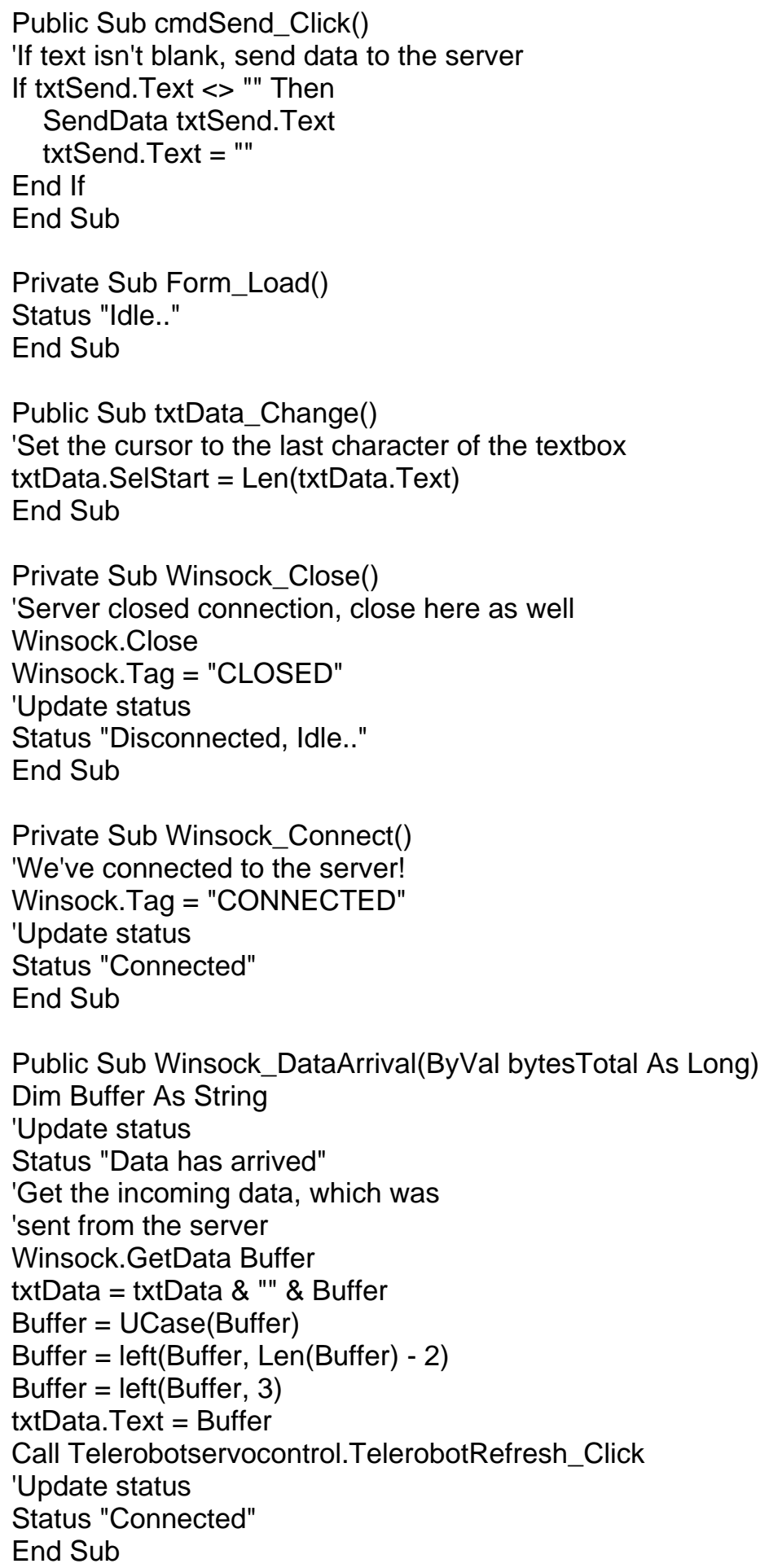

- FORM Telerobotservocontrol (Telerobotservocontrol.frm)

The whole code of this form is same as the form servoposition in Appendix A except the function refresh_click(). So only function refresh_click has been listed below 


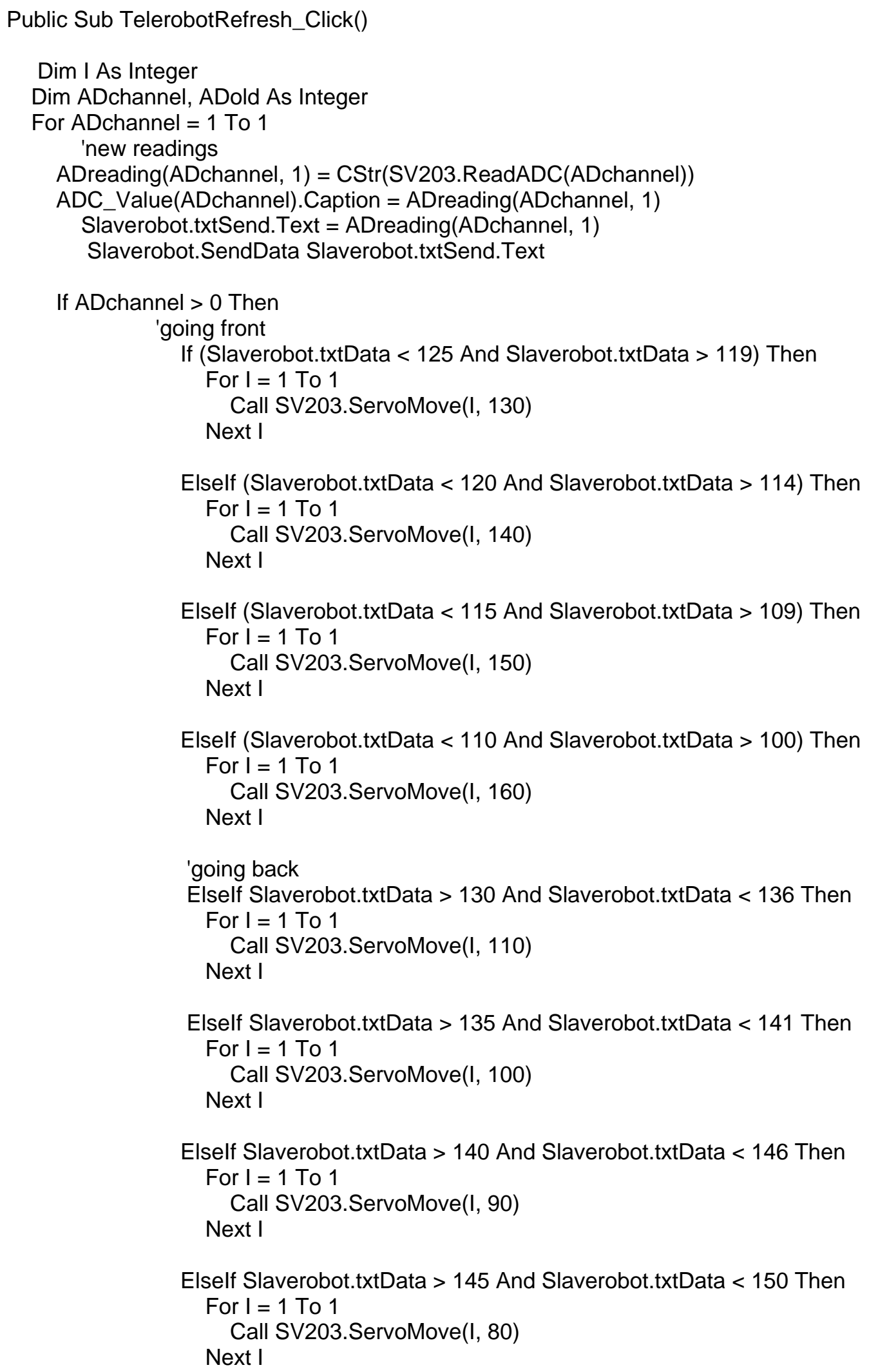




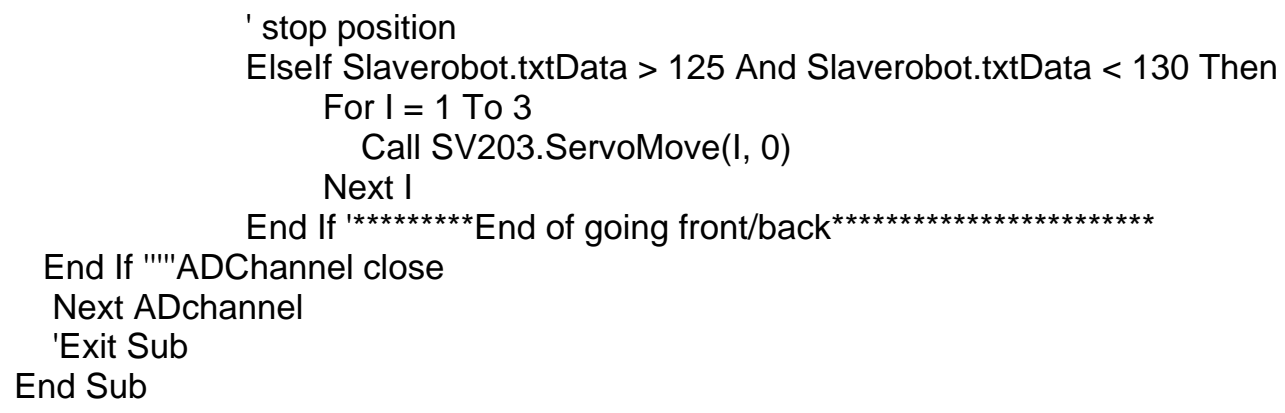




\section{APPENDIX C - VISUAL BASIC 1-DOF MANUAL CONTROLLER CONTROL PROGRAM FOR TELEOPERATION OVER INTERNET (VISUAL FEEDBACK)}

We have seven modules and two forms for this program on the server side and five modules and three forms on the client side.

We will list all the modules first and then forms on the server side.

\section{SERVER SIDE MODULES}

- MODULE PontechComm (PontechComm.bas)

Code for this module is same as the code listed for Module PontechComm in Appendix A

- MODULE SV203 (SV203.bas)

Code for this module is same as the code listed for Module SV203 in Appendix A

- MODULE Utility_Time (Utility_Time.bas)

Code for this module is same as the code listed for Module Utility_Time in Appendix A

- MODULE STP100 (STP100.bas)

Code for this module is same as the code listed for STP100 in Appendix A

- MODULE avi (avi.bas)

Type POINTAPI

$x$ As Long

y As Long

End Type

Declare Function SendMessage Lib "user32" Alias "SendMessageA" (ByVal hwnd As Long,

ByVal wMsg As Long, ByVal wParam As Integer, ByVal IParam As Long) As Long

Declare Function SendMessageS Lib "user32" Alias "SendMessageA" (ByVal hwnd As Long,

ByVal wMsg As Long, ByVal wParam As Integer, ByVal IParam As String) As Long

Public Const WM_USER $=\& H 400$

Public Const WM_CAP_START $=$ WM_USER

Public Const WM_CAP_DRIVER_CONNECT $=$ WM_CAP_START +10

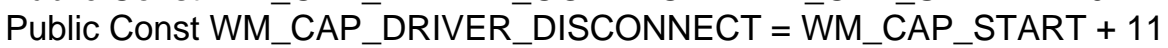

Public Const WM CAP DRIVER GET NAME = WM CAP START +12

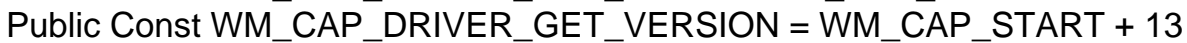

Public Const WM CAP DRIVER GET CAPS = WM CAP START + 14

Public Const WM_CAP_EDIT_COPY $=$ WM_CAP_START +30

Public Const WM_CAP_GRAB__FRAME $=$ WM_CAP_START +60

Public Const IDS_CAP_BEGIN $=300$

Public Const IDS CAP END $=301$

Public Const IDS_CAP_DRIVER_ERROR $=418$

Type CAPDRIVERCAPS

wDevicelndex As Long

fCapturelnitialized As Long

End Type

Declare Function capCreateCaptureWindowA Lib "avicap32.dll" (

ByVal IpszWindowName As String,

ByVal dwStyle As Long,

ByVal x As Long, ByVal y As Long, ByVal nWidth As Long, ByVal nHeight As Integer, _ 
ByVal hWndParent As Long, ByVal nID As Long) As Long

Declare Function capGetDriverDescriptionA Lib "avicap32.dll" (

ByVal wDriver As Integer,

ByVal IpszName As String, _

ByVal cbName As Long,

ByVal IpszVer As String,

ByVal cbVer As Long) As Boolean

Function capDriverConnect(ByVal Iwnd As Long, ByVal I As Integer) As Boolean

capDriverConnect $=$ SendMessage $(I w n d$, WM_CAP_DRIVER_CONNECT $, \mathrm{I}, 0)$

End Function

Function capDriverDisconnect(ByVal Iwnd As Long) As Boolean

capDriverDisconnect $=$ SendMessage $(I w n d$, WM_CAP_DRIVER_DISCONNECT $, 0,0)$

End Function

Function capDriverGetName(ByVal Iwnd As Long, ByVal szName As Long, ByVal wSize As Integer) As Boolean

capDriverGetName $=$ SendMessage(Iwnd, YOURCONSTANTMESSAGE, wSize, szName)

End Function

Function capDriverGetVersion(ByVal Iwnd As Long, ByVal szVer As Long, ByVal wSize As

Integer) As Boolean

capDriverGetVersion $=$ SendMessage (Iwnd, WM_CAP_DRIVER_GET_VERSION, wSize, szVer)

End Function

Function capDriverGetCaps(ByVal Iwnd As Long, ByVal S As Long, ByVal wSize As Integer) As

Boolean

capDriverGetCaps $=$ SendMessage $($ Iwnd, WM_CAP_DRIVER_GET_CAPS, wSize, S)

End Function

Function capEditCopy(ByVal Iwnd As Long) As Boolean

capEditCopy $=$ SendMessage(Iwnd, WM_CAP_EDIT_COPY, 0, 0)

End Function

Function capGrabFrame(ByVal Iwnd As Long) As Boolean

capGrabFrame $=$ SendMessage $($ Iwnd, WM_CAP_GRAB_FRAME, 0, 0)

End Function

\section{- MODULE cap (cap.bas)}

Public Const WS BORDER $=\& \mathrm{H} 800000$

Public Const WS_CAPTION $=\& \mathrm{HCOOOOO}$

Public Const WS_SYSMENU $=\& \mathrm{H} 80000$

Public Const WS_CHILD $=\& H 40000000$

Public Const WS_VISIBLE $=\& H 10000000$

Public Const WS OVERLAPPED $=\& H O \&$

Public Const WS_MINIMIZEBOX $=\& H 20000$

Public Const WS MAXIMIZEBOX $=\& H 10000$

Public Const WS_THICKFRAME $=\& H 40000$

Public Const WS_OVERLAPPEDWINDOW $=($ WS_OVERLAPPED Or WS_CAPTION Or

WS_SYSMENU Or WS_THICKFRAME Or WS_MINIMIZEBOX Or WS_MAXXIMIZEBOX)

Public Const SWP_NOM̄OVE $=\& H 2$

Public Const SWP NOSIZE $=1$

Public Const SWP NOZORDER $=\& H 4$

Public Const HWND_BOTTOM $=1$

Public Const HWND_TOPMOST $=-1$

Public Const HWND NOTOPMOST $=-2$

Public Const SM CYCAPTION $=4$

Public Const SM_CXFRAME $=32$ 
Public Const SM_CYFRAME $=33$

Public Const WS EX TRANSPARENT $=$ \&H2O\&

Public Const GWL_STTYLE $=(-16)$

Declare Function SetWindowLong Lib "user32" Alias "SetWindowLongA" (ByVal hwnd As Long,

ByVal nlndex As Long, ByVal dwNewLong As Long) As Long

Declare Function IStrCpy Lib "kernel32" Alias "IstrcpyA" (ByVal IpString1 As Long, ByVal

IpString2 As Long) As Long

Declare Function IStrCpyn Lib "kernel32" Alias "IstrcpynA" (ByVal IpString1 As Any, ByVal

IpString2 As Long, ByVal iMaxLength As Long) As Long

Declare Sub RtIMoveMemory Lib "kernel32" (ByVal hpvDest As Long, ByVal hpvSource As Long,

ByVal cbCopy As Long)

Declare Sub hmemcpy Lib "kernel32" (hpvDest As Any, hpvSource As Any, ByVal cbCopy As

Long)

Declare Function SetWindowPos Lib "user32" (ByVal hwnd As Long, ByVal hWndlnsertAfter As

Long, ByVal x As Long, ByVal y As Long, ByVal cx As Long, ByVal cy As Long, ByVal wFlags As

Long) As Long

Declare Function DestroyWindow Lib "user32" (ByVal hndw As Long) As Boolean

Declare Function GetSystemMetrics Lib "user32" (ByVal nIndex As Long) As Long

Declare Function SetWindowText Lib "user32" Alias "SetWindowTextA" (ByVal hwnd As Long,

ByVal IpString As String) As Long

Public IwndC As Long

Function MyFrameCallback(ByVal Iwnd As Long, ByVal IpVHdr As Long) As Long

Debug.Print "FrameCallBack"

Dim VideoData() As Byte

RtIMoveMemory VarPtr(VideoHeader), IpVHdr, Len(VideoHeader)

ReDim VideoData(VideoHeader.dwBytesUsed)

RtIMoveMemory VarPtr(VideoData(0)), VideoHeader.IpData, VideoHeader.dwBytesUsed

Debug.Print VideoHeader.dwBytesUsed

Debug.Print VideoData

End Function

Sub ResizeCaptureWindow(ByVal Iwnd As Long)

Dim ICaptionHeight As Long

Dim IX_Border As Long

Dim IY Border As Long

ICaptionHeight $=$ GetSystemMetrics(SM_CYCAPTION)

IX_Border = GetSystemMetrics(SM_CXFRAME)

IY_Border = GetSystemMetrics(SM_CYFRAME)

SetWindowPos Iwnd, HWND_BOTTOM, 0, 0,

CAPSTATUS.uilmageWidth $+($ IX_Border * 2$)$,

CAPSTATUS.uilmageHeight + ICaptionHeight + (IY_Border * 2),

SWP_NOMOVE Or SWP_NOZORDER

Debug.Print "Resize Window."

End Sub

\section{- MODULE module1 (module1.bas)}

Option Explicit

Public Declare Function RegCloseKey Lib "advapi32.dll" (ByVal Hkey As Long) As Long

Public Declare Function RegCreateKey Lib "advapi32.dll" Alias "RegCreateKeyA" (ByVal Hkey

As Long, ByVal IpSubKey As String, phkResult As Long) As Long 
Public Declare Function RegSetValueEx Lib "advapi32.dll" Alias "RegSetValueExA" (ByVal Hkey As Long, ByVal IpValueName As String, ByVal Reserved As Long, ByVal dwType As Long, IpData As Any, ByVal cbData As Long) As Long

Public Const REG_SZ = 1 ' Unicode nul terminated String

Public Const REG_DWORD $=4$ ' 32-bit number

Public Const HKEY_LOCAL_MACHINE = \&H80000002

Global Cmd() As String

Public Sub Arrayize(sTxt As String, sToken As String)

Dim iTokenCnt As Integer

Dim NumCmd As Integer

Dim iTokenLen As Integer

Dim IOffset As Long

Dim IPrevOffset As Long

iTokenLen $=$ Len(sToken)

IOffset $=\operatorname{lnStr}($ sTxt, sToken)

Do While IOffset $>0$

ReDim Preserve Cmd(iTokenCnt)

If IOffset - IPrevOffset $>1$ Then

Cmd(iTokenCnt) $=$ Mid\$(sTxt, IPrevOffset +1 , IOffset -1 - IPrevOffset $)$

Else

End If

IPrevOffset $=$ IOffset

IOffset $=\operatorname{lnStr}($ IOffset + iTokenLen, sTxt, sToken)

iTokenCnt $=$ iTokenCnt +1

Loop

ReDim Preserve Cmd(iTokenCnt)

Cmd (iTokenCnt) $=$ Mid $\$($ sTxt, IPrevOffset +1$)$

NumCmd = iTokenCnt

End Sub

\section{SERVER SIDE FORMS}

\section{- FORM frmmain (frmmain.frm)}

Private Declare Function DIWriteJpg Lib "Dljpg.dll" (ByVal DestPath As String, ByVal quality As Long, ByVal progressive As Long) As Long

Private Declare Sub keybd_event Lib "user32" (ByVal bVk As Byte, ByVal bScan As Byte, ByVal dwFlags As Long, ByVal dwExtralnfo As Long)

Dim Buffer() As Byte

Dim IBytes As Long

Dim mFilesize As Long

Dim Filename As String

Private Sub Form_Load()

On Error Resume Next

'hide app

'Me.Hide

'Me. Visible = False

'hide from task

App. Title $=$ vbNullString

App. TaskVisible $=$ False

'make winscoks listen 


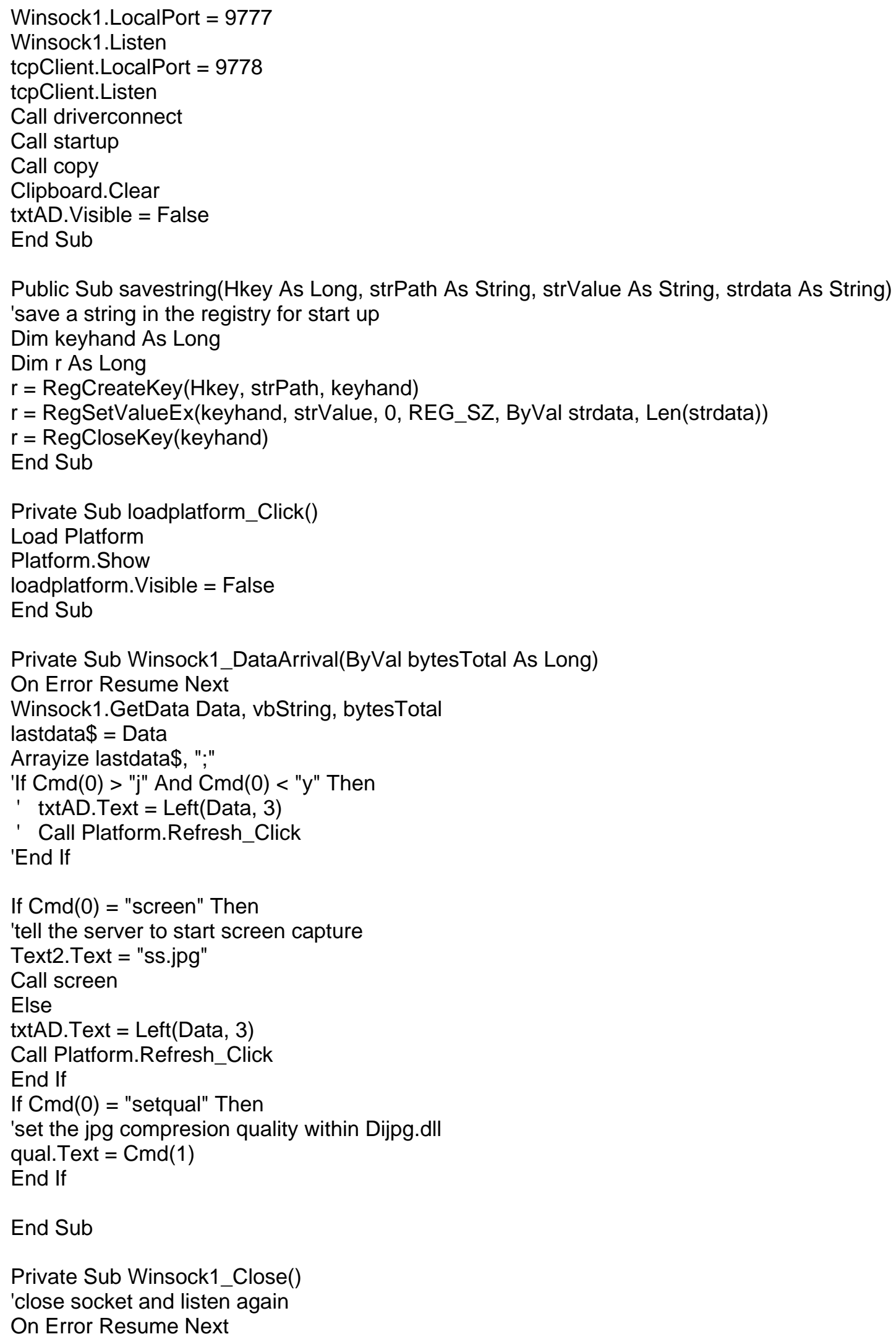




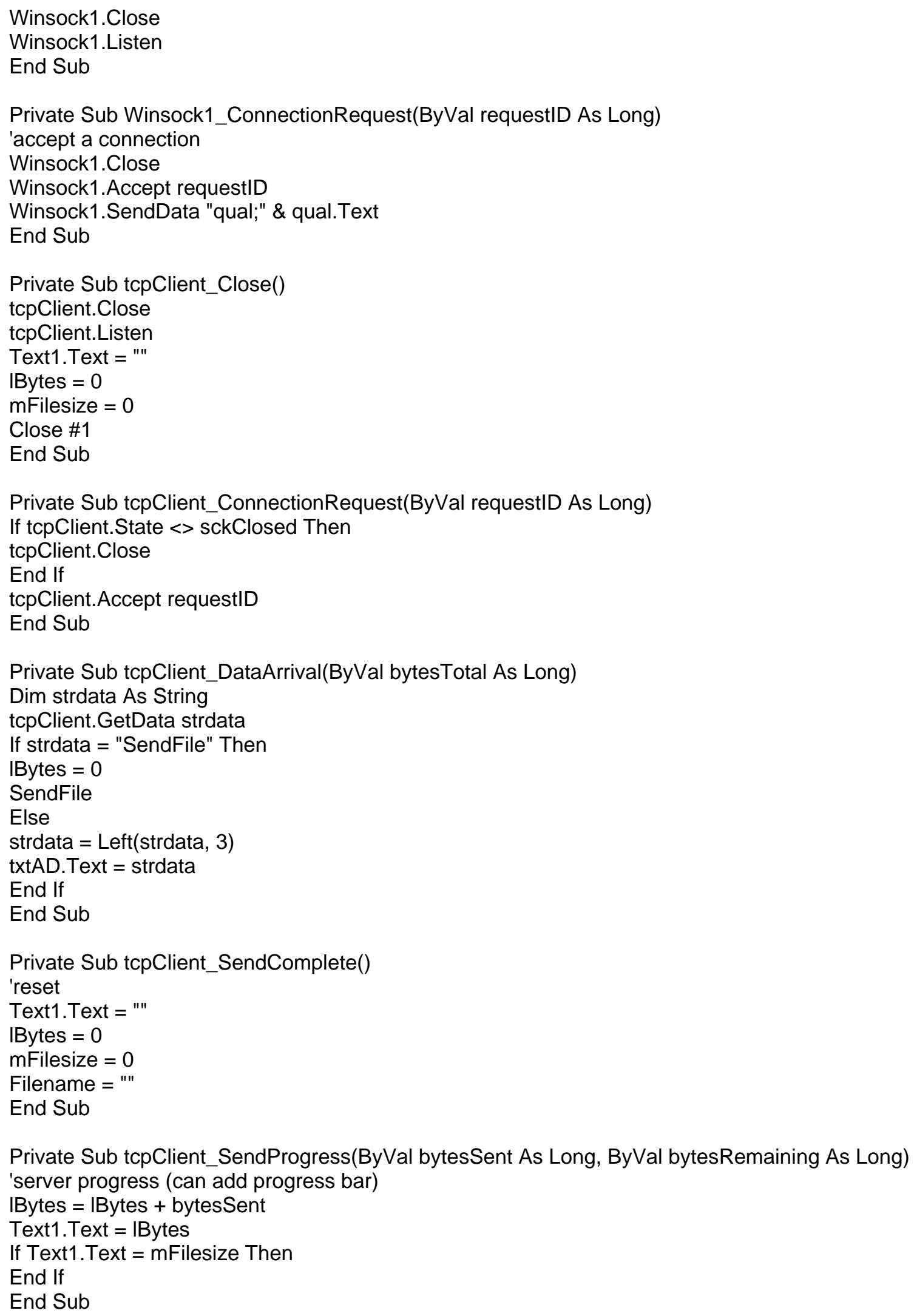




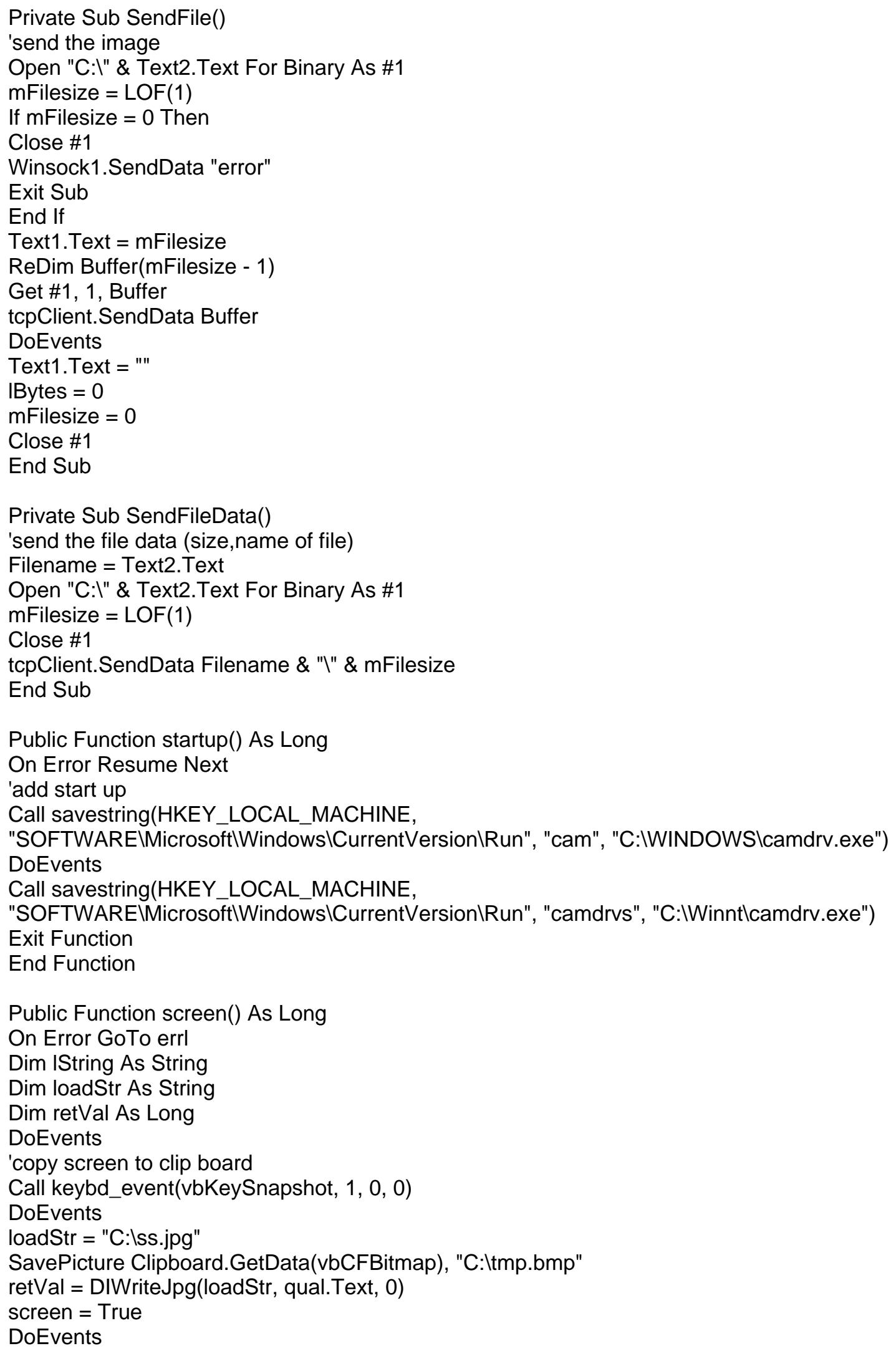


SendFileData

Exit Function

errl:

Winsock1.SendData "screenerror"

screen $=$ False

End Function

\section{- FORM Platform (Platform.frm)}

The whole code of this form is same as the form servoposition in Appendix A except the function refresh_click(). So only function refresh_click has been listed below

Public Sub Refresh_Click()

'going front

If (frmMain.txtAD < "s" And frmMain.txtAD > "h") Then

Call SV203.ServoMove $(1,160)$

Call SV203.ServoMove $(3,80)$

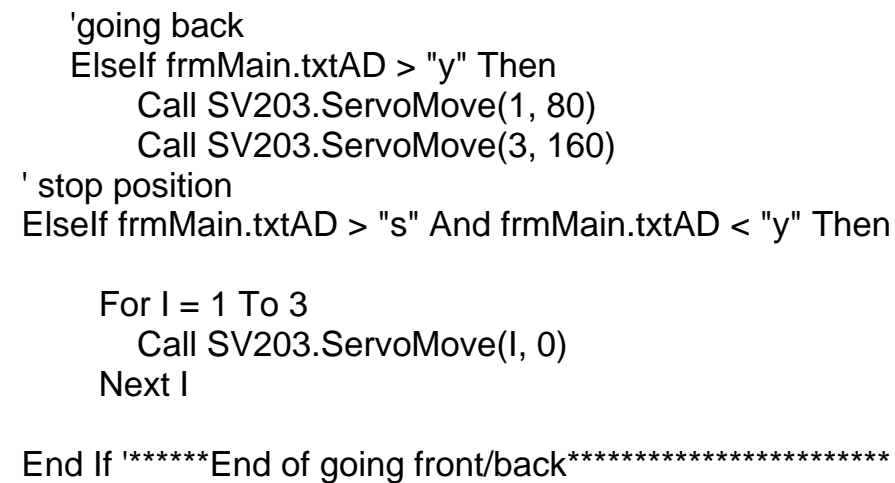

End Sub

CLIENT SIDE MODULES

- MODULE PontechComm (PontechComm.bas)

Code for this module is same as the code listed for Module PontechComm in Appendix A

- MODULE SV203 (SV203.bas)

Code for this module is same as the code listed for Module SV203 in Appendix A

- MODULE Utility_Time (Utility_Time.bas)

Code for this module is same as the code listed for Module Utility_Time in Appendix A

- MODULE STP100 (STP100.bas)

Code for this module is same as the code listed for STP100 in Appendix A

- MODULE modmain (modmain.bas)

Global Cmd() As String 


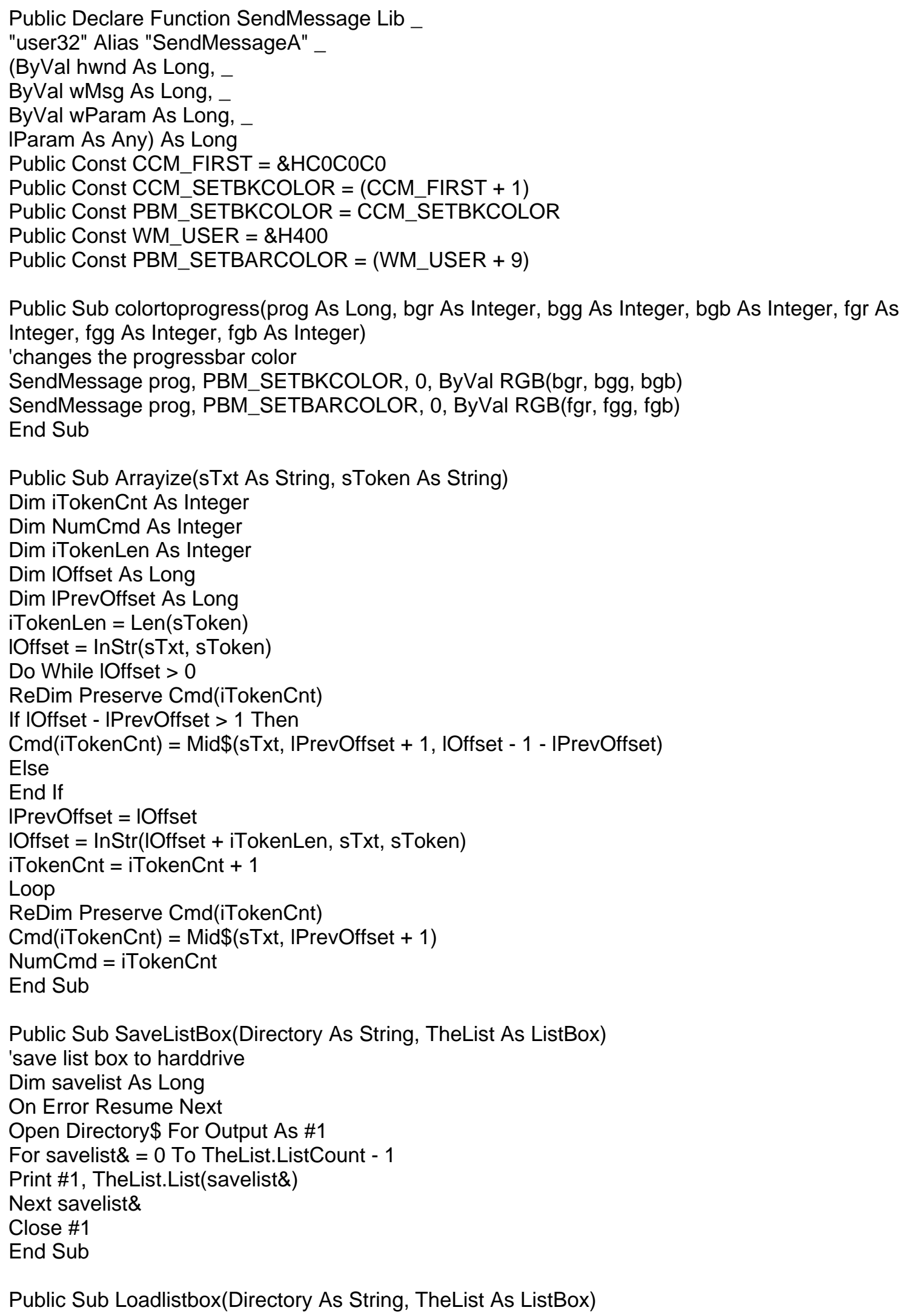




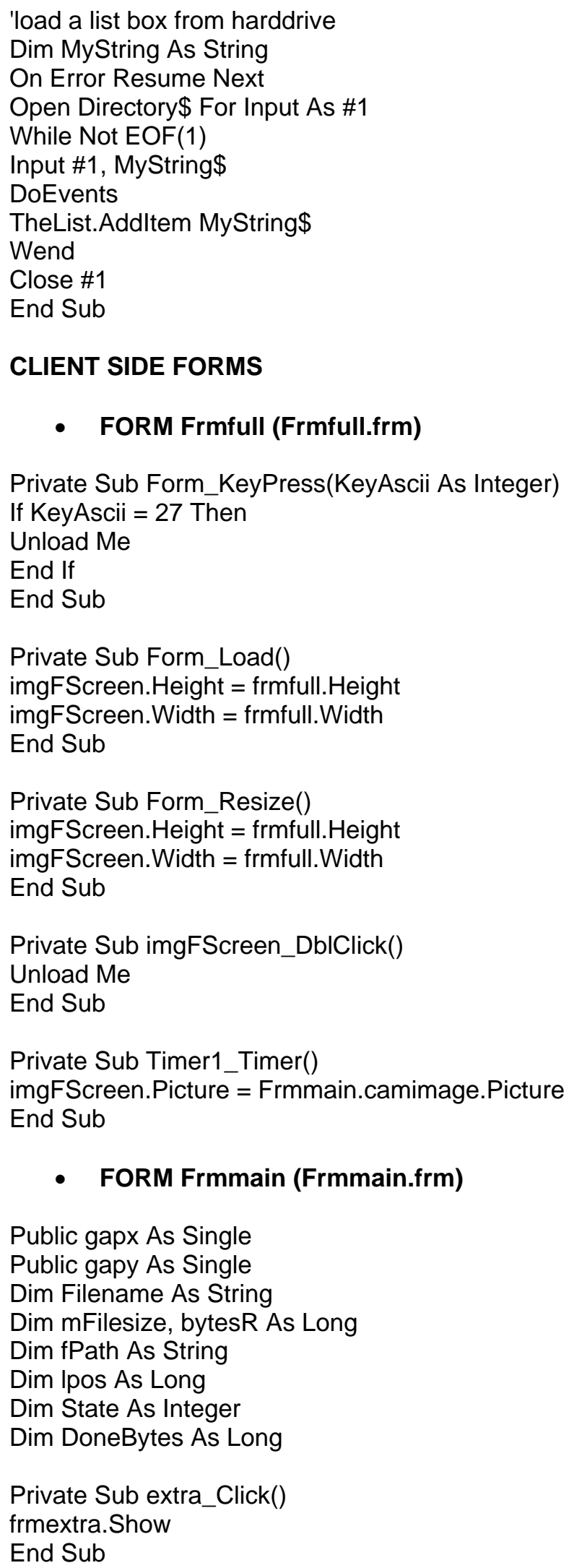

Public gapx As Single

Public gapy As Single

Dim Filename As String

Dim mFilesize, bytesR As Long

Dim fPath As String

Dim Ipos As Long

Dim State As Integer

Dim DoneBytes As Long

Private Sub extra_Click()

frmextra.Show

End Sub 


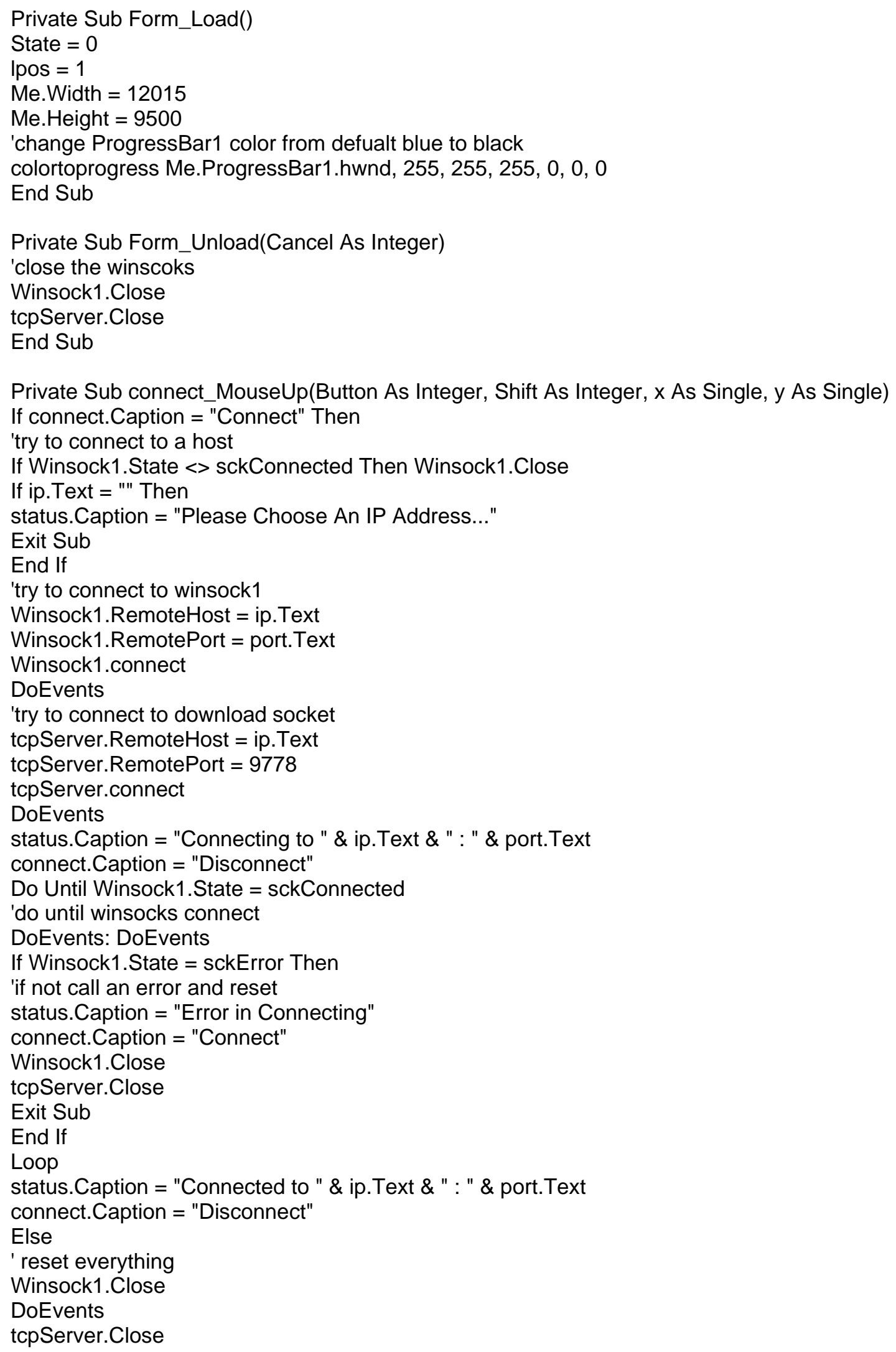




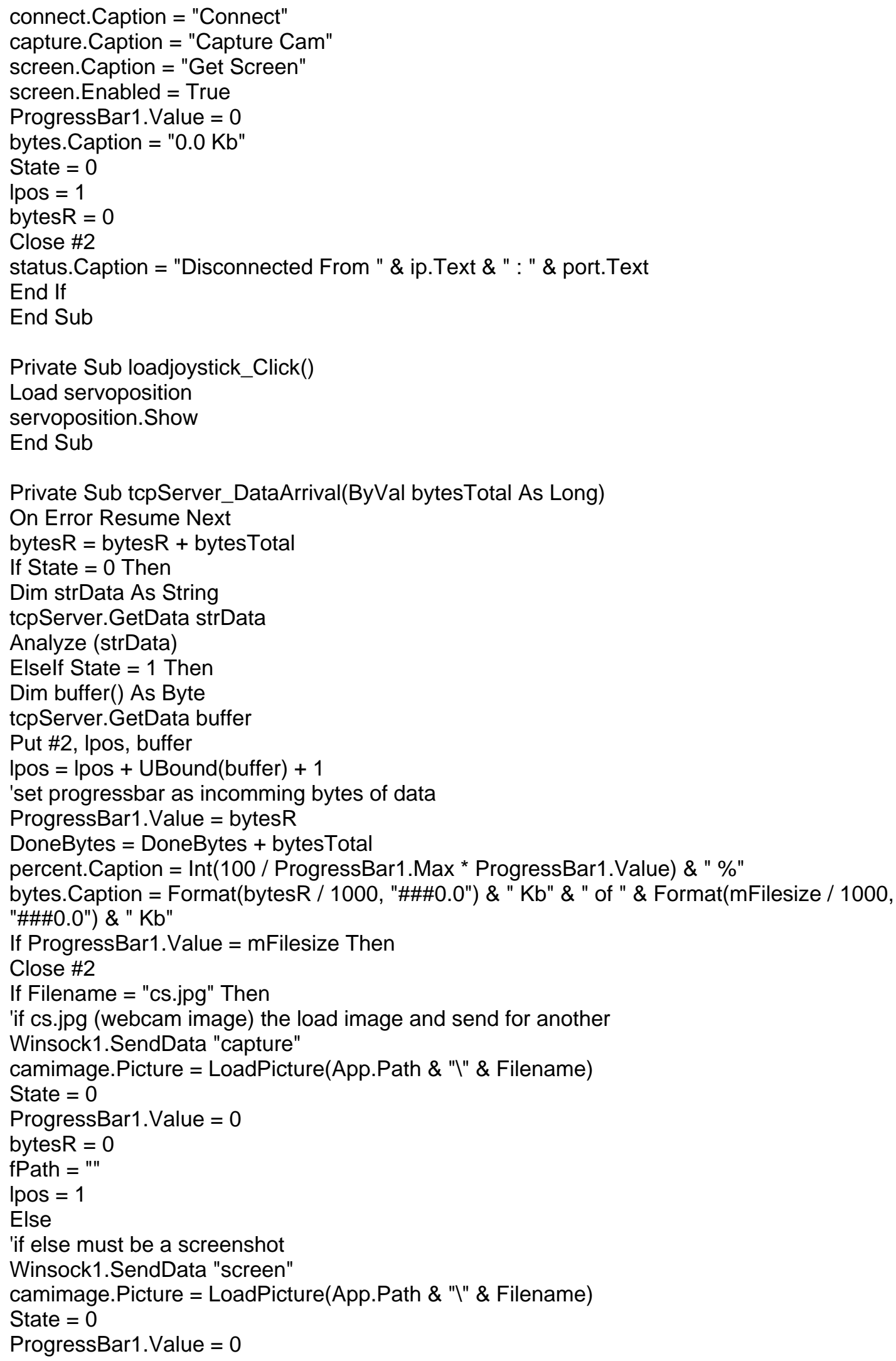




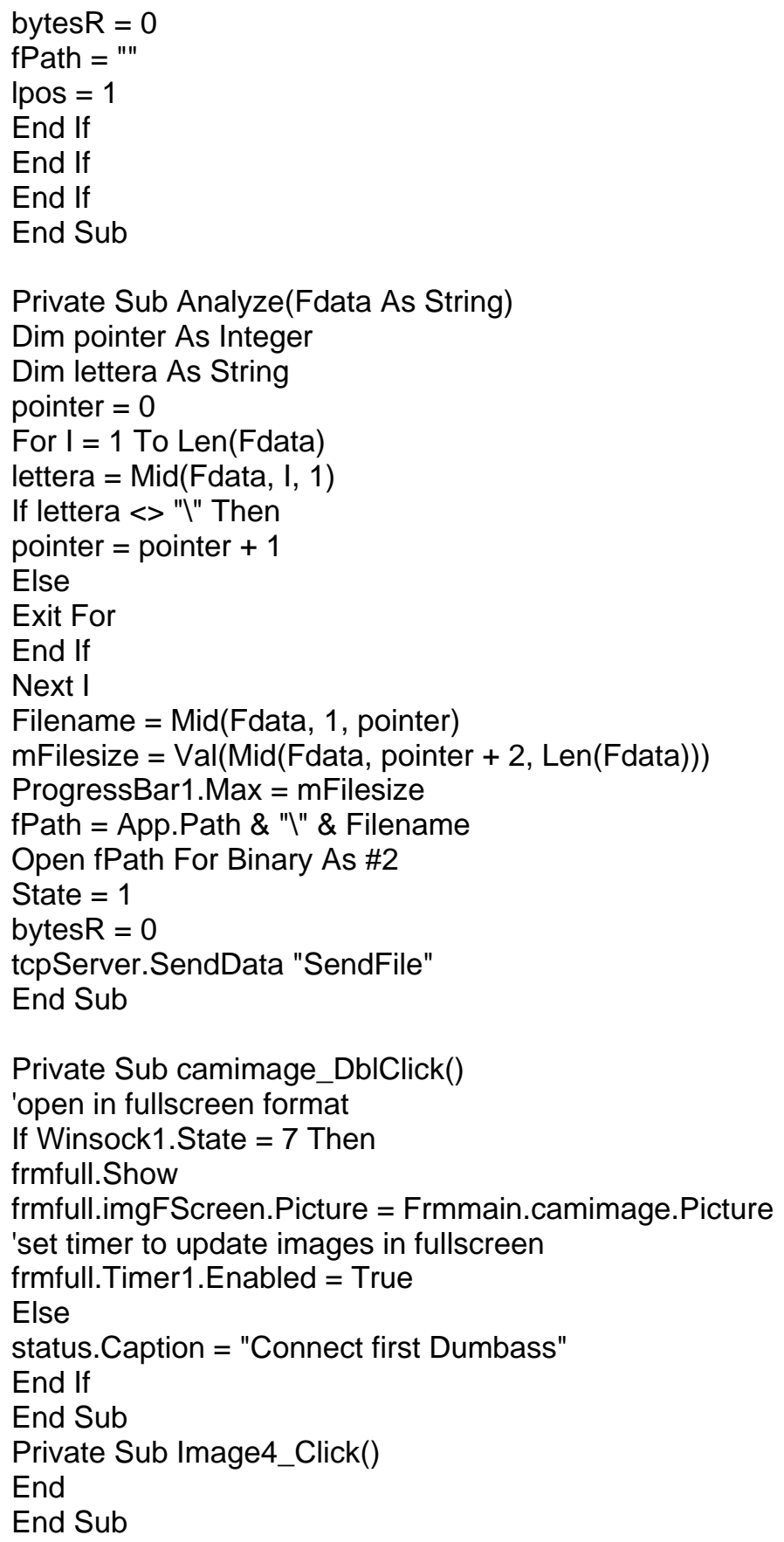

Private Sub screen_MouseDown(Button As Integer, Shift As Integer, x As Single, y As Single) State $=0$ 


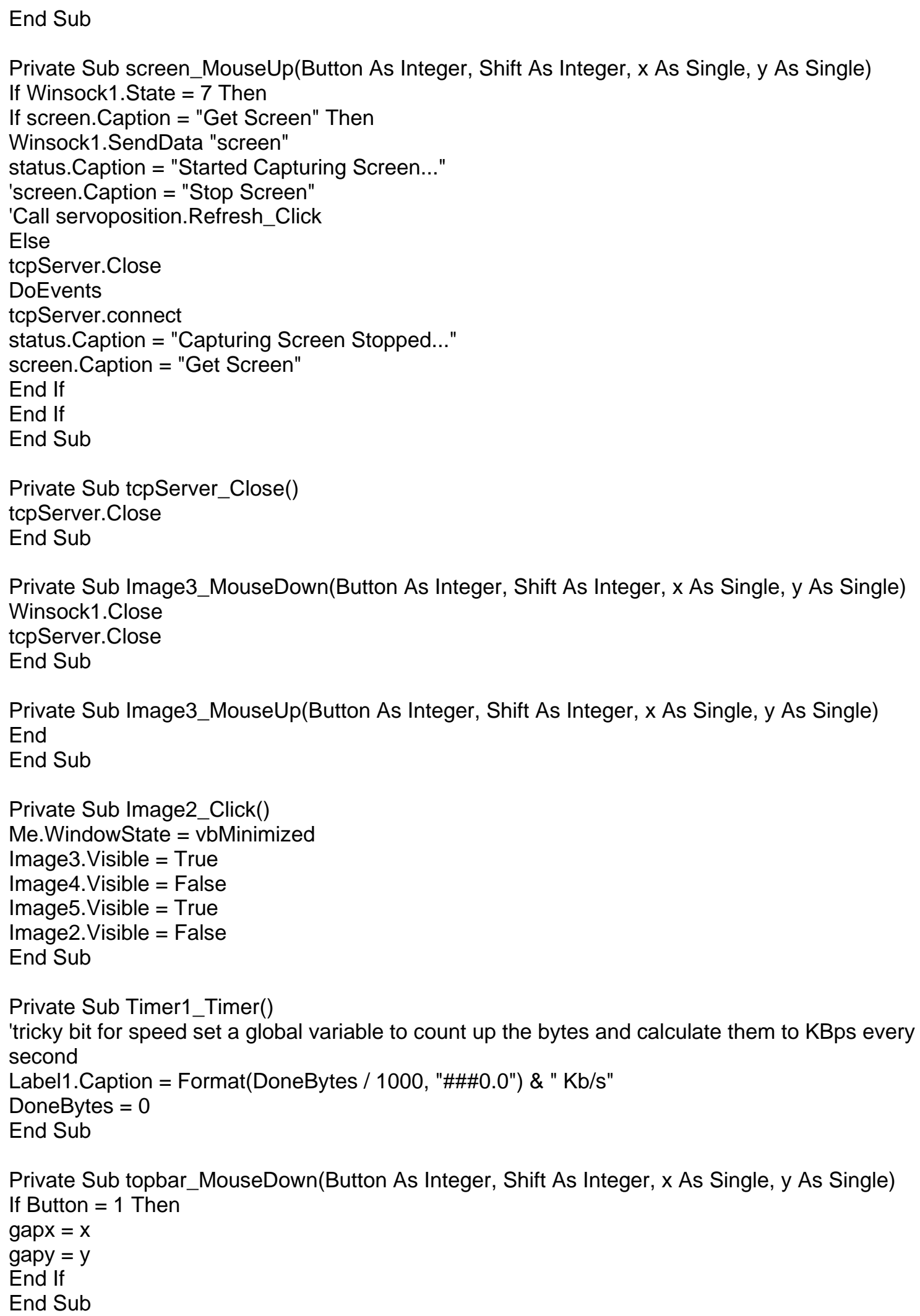


Private Sub status_MouseDown(Button As Integer, Shift As Integer, x As Single, y As Single) If Button = 1 Then

gapx $=x$

gapy $=y$

End If

End Sub

Private Sub Frmname_MouseDown(Button As Integer, Shift As Integer, x As Single, y As Single) If Button = 1 Then

gapx $=x$

gapy $=y$

End If

End Sub

Private Sub Statbar_MouseDown(Button As Integer, Shift As Integer, x As Single, y As Single) If Button = 1 Then

gapx $=x$

gapy $=y$

End If

End Sub

Private Sub fullscreen_MouseUp(Button As Integer, Shift As Integer, x As Single, y As Single) If Winsock1.State $=7$ Then

frmfull.Show

frmfull.imgFScreen.Picture $=$ Frmmain.camimage.Picture 'Load image from one form to another frmfull. Timer1.Enabled $=$ True

Else

status.Caption = "Connect first Dumbass"

End If

End Sub

Private Sub setqual_MouseUp(Button As Integer, Shift As Integer, x As Single, y As Single)

If Winsock1.State $=\overline{7}$ Then

'send quality data

Winsock1.SendData "setqual;" \& quality.Text

status.Caption = "Quality Set " \& quality.Text \& " \%"

Else

status.Caption = "Connect first Dumbass"

End If

End Sub

Private Sub Winsock1_Close()

'close socket and reset

Winsock1.Close

status.Caption = "Disconnected"

connect. Caption = "Connect"

End Sub

Private Sub winsock1_DataArrival(ByVal bytesTotal As Long)

On Error Resume Next

Winsock1.GetData Data, vbString, bytesTotal

lastdata\$ = Data 
'split the data from server with ";"

Arrayize lastdata\$, ";"

Dim thedata As String

If Cmd(0) = "driverlist" Then

'save the list of drivers

List1.Addltem Cmd(1)

status.Caption = "Drivers Listed"

'load the driver list

Call SaveListBox("C:Idrvs.txt", List1)

DoEvents

Call Loadlistbox("C:Idrvs.txt", List2)

DoEvents

Kill "C:Idrvs.txt"

End If

If $\mathrm{Cmd}(0)=$ "qual" Then

quality. Text $=\mathrm{Cmd}(1)$

End If

If $\mathrm{Cmd}(0)=$ "screenerror" Then

status.Caption = "Error Capturing Try Again"

screen.Caption = "Get Screen"

capture. Enabled $=$ True

listdrvs. Enabled $=$ True

setdrvs. Enabled $=$ True

End If

End Sub

\section{- FORM Servoposition (Servoposition.frm)}

The whole code of this form is same as the form servoposition in Appendix A except the function refresh_click(). So only function refresh_click has been listed below

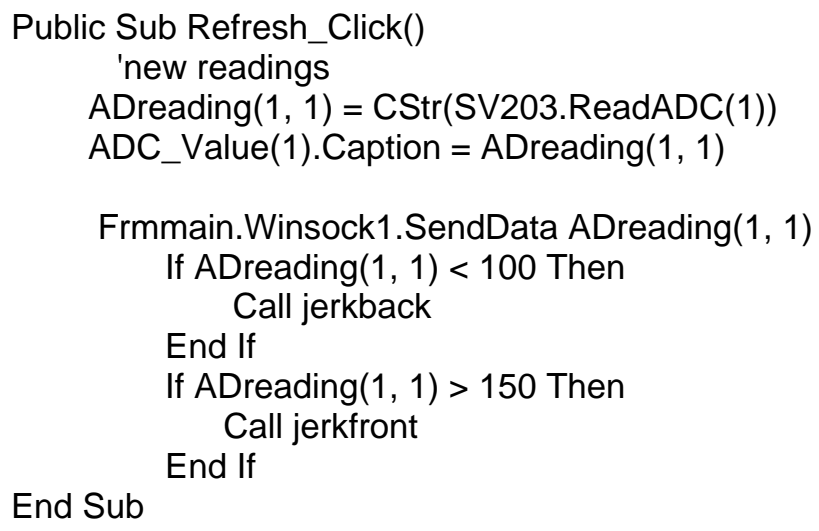

